\title{
Malacologie alpestre
}

\author{
PAR
}

\section{Jean PIAGE T}

Avec la planche $\mathbf{1}^{\prime}$.

Ce travail est le résultat de recherches faites dans cinq vallées des Alpes. J'ai dressé le catalogue de toutes les espèces de Mollusques que j'ai pu rencontrer, en notant soigneusement les conditions de trouvaille et l'altitude du lieu de récolte, le tout pour servir à des études plus générales sur les Mollusques suisses et leur distribution hypsométrique. Ce catalogue n'a en aucune façon la prétention d'être complet. C'est un simple essai, qui pourra peut-être servir de point de départ à des recherches futures.

Mollusques récoltés dans le vallon des Plans, au-dessus de Bex (Vaud).

On trouve déjà dans le Catalogue de Charpentier (1837), qui, comme on le sait, fut longtemps directeur des Salines de Bex, quelques renseignements sur ce sujet, mais en somme fort peu de choses. J'ai récolté jusqu'à présent 46 espèces, aux environs immédiats des Plans, depuis Frenières, dans les gorges de l'Avançon, du côté des Parts et jusqu'à la limite supérieure

Rev. Suisse de Zool. T. 21. 1913. 
des forêts, au-dessus du jardin botanique de Pont-de-Nant, à la Larze et aux Planaires.

Les Plans, à l'altitude de $1100^{\mathrm{m}}$ environ, sont situés près de la limite entre les Mollusques de la plaine et les espèces franchement alpines : on y rencontre encore, à $1200^{\mathrm{m}}$ environ, les Helicodonta obvoluta et Patula rotundata qui vivent en compagnie de $H$. holoserica et $P$. ruderata; les Clausilia laminata et fimbriata se trouvent souvent ensemble sur le mème tronc, etc. Ce n'est qu'à partir de Pont-de-Nant, à 1250-1300 ${ }^{\mathrm{m}}$ environ, que les espèces de la plaine font défaut : c'est la région supérieure des forèts.

\section{GASTROPODA}

\section{S.-Gl. PULMONATA}

Fam. Vitrinidae.

Gen. Limax Müll.

\section{Limax (Heynemannia) maximus L.}

Hab. ${ }^{1}$ Sous les feuilles mortes, sur les troncs, sous les morceaux de bois, les écorces, se dissimulant partout avec leurs taches noires ou brunes.

D. h. ${ }^{1}$ Commune dans toutes les forèts.

D. v. ${ }^{1}$ Jusqu'à 1500-1600"w au Pont de-Nant.

On trouve les deux variétés suivantes:

a) Var. cinerea (List.), qui ne dépasse pas $1200^{\mathrm{m}}$.

b) Var. cinereo-niger (Wolff.), dans toute la région.

2. Limax (Heynemannia) tenellus Nils.

Hab. Sous le bois pourri, les écorces, les pierres.

${ }^{1}$ Hab. = Habitat ; D. h. = Distribution horizontale; D. v. = Distribution verticale. 
D. h. Assez fréquente dans les forêts, surtout près de Pontde-Nant.

D. v. Jusqu'à 1500-1600 ${ }^{\mathrm{m}}$. Elle surpasse ordinairement en altitude l'espèce précédente.

Gen. Agriolimax Simroth.

3. Agriolimax agrestis (L.).

Hab. Dans les champs (au pied des plantes, etc.) et les forêts (sous les pierres et les écorces).

D. h. Très commune partout, mais moins fréquente dans les forêts.

D. v. Jusqu’à $1600^{\mathrm{m}}$.

Gen. Vitrina Drap.

4. Vitrina (Semilimax) diaphana Drap.

Hab. Sous les feuilles, le bois mort, les pierres, l'écorce, etc.

D. h. Dans les forèts, du còté de Pont-de-Nant.

D. v. Elle ne s'élève que jusqu'à environ $1500-1600^{\mathrm{m}}$.

Gen. Hyalina Fér.

5. Hyalina (Euhyalina) glabra (Stud.).

Hab. Sous les pierres et le bois mort.

D. h. Dans les forèts de Pont-de-Nant.

D. v. Elle atteint la limite supérieure des forèts (Pont-de-Nant à $\left.1600^{\mathrm{m}}\right)$.

Forma minor. - $\left(11^{\mathrm{mm}}\right.$ de grand diamètre). Forêt des Plans.

6. Hyalina (Euhyalina) villae Mort. in Cless.

Hab. Sous les pierres.

D. h. Forêts de Pont-de-Nant.

D. v. Je l'ai trouvée à près de $1200^{\mathrm{m}}$. 
Forma minor. - Une dizaine de millim. de diamètre; à part cela bien caractéristique (fig. 3).

\section{Hyalina (Polita) nitens (Mich.).}

Hab. Sous les pierres, le bois mort et pourri, les feuilles, l'écorce, etc.

D. h. Très commune dans toutes les forêts.

D. v. Jusqu'à $1600^{\mathrm{m}}$ au Pont-de-Nant.

8. Hyalina (Polita) pura (Ald.).

Hab. Comme les espèces précédentes.

D. h. Commune dans les forêts.

D. v. Elle atteint $1500^{\mathrm{m}}$ au-dessus de Pont-de-Nant. CharpenTIEr la cite au même endroit, à la page 13, sous le nom de H. nitidula, qui est une espèce différente.

\section{Hyalina (Polita) radiatula Gray.}

Hab. Comme les espèces précédentes.

D. h. Comme la $H$. pura, mais moins commune.

D. v. Jusqu'à $1500-1600^{\mathrm{m}}$ environ.

Gen. Crystallus Lowe.

10. Crystallus diaphanus (Stud.).

Hab. Sous le bois pourri et les feuilles mortes.

D. h. Très commun dans les forèts.

D. v. Jusqu'à environ $1500-1600^{\mathrm{m}}$.

\section{Fam. Naninidae.}

Gen. Euconulus Reinh.

\section{Euconulus fulvus (Müll.).}

Hab. Sous le bois mort et sous la mousse.

D. h. Très répandı dans les forèts.

D. v. Comme les espèces précédentes. 
Fam. Arionidae.

Gen: Arion Fér.

12. Arion empiricorum Fér.

Hab. Sous les pierres, le bois pourri, l'écorce, etc.

D. h. Très commun dans les champs, moins dans les forèts; Gorges de l'Avançon, Frénières et les Plans.

D. v. Région inférieure des forèts, jusqu'à $1200^{\mathrm{m}}$ environ.

13. Arion subfuscus Drap.

Hab. Comme l'A. empiricorum.

D. h. Le plus souvent dans les forèts, répandu dans tout le vallon.

D. v. Jusqu'à $1500-1600 \mathrm{~m}$.

\section{Arion hortensis Fér.}

Hab. Dans les champs et les forèts, sur les vieux troncs, sous la mousse, les pierres, etc.

D. h. Commun partout.

D. v. Régions supérieure et inférieure des forêts, jusqu'à environ $1600^{\mathrm{m}}$.

\section{Fam. Patulidae.}

\section{Gen. Patula Held.}

\section{Patula (Discus) rotundata (Müll.).}

Hab. Sous les pierres et le bois mort.

D. h. Frénières, Gorges de l'Avançon, les Plans jusqu'un peu au-dessus du village.

D. v. Aux Plans, je ne l'ai rencontrée que jusqu'à $1200^{\mathrm{m}}$ environ. 


\section{Patula (Discus) ruderata (Stud.).}

Hab. Sous les pierres et le bois mort; sur les vieux troncs et les blocs moussus.

D. h. Dans les forêts au-dessus des Plans; Pont-de-Nant, la Larze.

D. v. $1200-1600^{\mathrm{m}}$ environ.

Remarque: Cette espèce vit en compagnie de la précédente et des Helicodonta obvoluta et holoserica, un peu au-dessus des Plans, à environ $1200^{\mathrm{m}}$; mais à mesure que l'on s'élève sur le chemin de Pont-de-Nant, les $H$. obvoluta et $P$. rotundata disparaissent et, au-dessus du Jardin botanique, on ne trouve plus que les formes alpines $\left(1250^{\mathrm{m}}\right)$. Les individus de Patula ruderata varient beaucoup sous le rapport de la couleur, qui passe du brun foncé au vert foncé; cependant je n'ai pas trouvé la var. viridana de Rofriaen, qui a une coloration vert clair et un test très mince.

Gen. Pyramidula Fitz.

17. Pyramidula rupestris (Drap.).

Hab. Contre les rochers. La coquille est généralement recouverte de dépòts grisâtres dont la couleur se confond parfaitement avec celle de la pierre environnante.

D. h. Le long de la route entre Frénières et les Plans; Gorges de l'Avançon, Pont-de-Nant.

D. v. Jusque vers $1250^{\mathrm{m}}$ au Pont-de-Nant.

\section{Fam. Eulotidae.}

Gen. Eulota Htm.

\section{Eulota fruticum (Müll.).}

Hab. Dans les prairies, au bas des haies, sous les buissons.

D. h. Environs des Plans. 
D. v. Environ $950-1200^{\mathrm{m}}$.

Les exemplaires des Plans sont normaux; l'altitude n'a encore aucun effet sur leur taille.

\section{Fam. Helicidae.}

S.-Fam. Helicodontinae.

Gen. Helicodonta Risso.

19. Helicodonta (Trigonostoma) obvoluta (Müll.).

Hab. Sous les pierres et le bois mort.

D. h. Dans toutes les forèts jusqu'un peu au-dessus des Plans: Gorges de l'Avançon, etc.

D. v. Mème distribution hypsométrique que la Patula rotundata.

\section{Helicodonta (Trigonostoma) holoserica (Stud.).}

Hab. Sous les pierres, souvent mème assez profondément; parmi les éboulis.

D. h. Au-dessus des Plans et Pont-de-Nant.

D. v. De mème que la Patula ruderata.

$$
\begin{aligned}
& \text { S.-Fam. Fruticicolinae. } \\
& \text { Gen. Fruticicola Held. }
\end{aligned}
$$

21. Fruticicola (Fruticicola s. str.) sericea (Drap.).

Hab. Sous le bois mort et les pierres.

D. h. Dans toutes les forêts.

D. v. Jusqu'à $1500^{\mathrm{m}}$ environ. 
22. Fruticicola (Fruticicola) villosa (Drap.).

Hab. Sous le bois pourri, le feuillage mort ou sur les feuilles de certaines plantes (Petasiles, etc.).

D. h. Extrèmement commune dans toutes les forèts, seulement jusqu'à une certaine altitude.

D. v. Région inférieure des forèts. Je ne l'ai trouvée, près de Pont-de-Nant, que jusqu'à 1200-1250m environ.

\section{Fruticicola (Monacha) incarnata (Müll.).}

Hab. Sous le feuillage mort.

D. h. Gorges de l'Avançon, les Plans; très disséminée.

D. v. Région inférieure des forèts, jusqu'à environ $1200^{\mathrm{m}}$.

S.-Fam. Campylaeinae.

Gen. Isognomostoma Fitz.

24. Isognomostoma personatum (Lam.).

Hab. Sous les pierres et le bois mort; elle se confond, par sa couleur brune, avec les troncs pourris.

D. h. Forèts des Gorges de l'Avançon, des Plans et de Pontde-Nant.

D. v. Jusqu'à $1500^{\mathrm{m}}$ environ; elle n'atteint pas la limite supérieure des forèts.

Gen. Chilotrema Leach.

25. Chilotrema lapicida (L.).

Hab. Ordinairement contre les rochers, mais aussi sous les pierres et le bois pourri.

D. h. Gorges de l'Avancon, les Plans et Pont-de-Nant.

D. v. Jusqu'à $1250^{\mathrm{m}}$. 
Gen. Arianta Leach.

26. Arianta arbustorum (L.).

Hab. Dans l'herbe, sous le bois et le feuillage mort, etc.

D. h. Très commune dans les champs et les forèts.

D. v. Le type ne monte pas très haut: il atteint au-dessus des Plans environ $1250^{\mathrm{m}}$.

Il présente diverses variations de forme et de couleur :

F. trochoidalis, depressa, minor, etc.

Mut. plus minusve pallida, maculata, effasciata, etc.

$$
\text { Var. alpicola Charp. }
$$

D. h. A partir de Pont-de-Nant.

D. v. $1250-1600^{\mathrm{m}}$.

S.-Fam. Helicinae.

Gen. Helix L., s. str.

27. Helix pomatia L.

Hab. Dans les champs et sur la lisière des forèts ; rarement à l'intérieur des bois.

D. h. Dans tout le fond de la vallée de Frénières au Pont-dede-Nant.

D. v. Jusqu'à $1250^{\mathrm{m}}$ environ.

Diverses variations de forme :

F. normalis (var. rustica $\mathrm{Htm}$.).

F. compacta (Htm.) (F. globosa Godet).

F. elevata God. (var. gessneri Htm.?).

$$
\text { Gen. Tachea Leach. }
$$

28. Tachea nemoralis (L.).

Hab. Contre les rochers et sous le feuillage mort.

D. h. Le bas des Gorges de l'Avançon. 
D. v. Seulement jusqu'à environ $1000^{\mathrm{m}}$.

Elle présente le plus souvent la couleur jaune, avec une ou deux) ou sans fascies foncées au-dessous de la coquille.

\section{Tachea hortensis (Müll.).}

Hab. Comme l'espèce précédente, mais plus fréquemment contre les rochers.

D. h. Gorges de l'Avançon. Charpentier signale sa var. montana ("couleur de chair uniforme») dans les montagnes audessus de Bex.

D. v. Jusqu'à environ $1100^{\mathrm{m}}$, un peu plus haut que l'espèce précédente.

\section{Tachea sylvatica (Drap.).}

Hab. Contre les rochers, ayant plus ou moins la couleur environnante.

D. h. Le long de la route entre Frénières et les Plans; Gorges de l'Avancon.

D. v. Jusqu'à environ $1150-1200^{\mathrm{m}}$.

Var. montana Stud.

D. h. Pont-de-Nant.

D. v. A partir d'environ $1200-1250^{\mathrm{m}}$ jusqu'à $1500^{\mathrm{m}}$ environ.

\section{S.-Fam. Xerophilinae. \\ Gen. Xerophila Held.}

31. Xerophila (Xerophila s. str.) ericetorum (Müll.).

Hab. Dans les champs en plein soleil, le long des tiges de céréales ou dans l'herbe.

D. h. Entre les Plans et les Parts.

D. v. Environ $1000-1300^{\mathrm{m}}$. 


\section{Fam. Buliminidae.}

Gen. Buliminus Ehr.

32. Buliminus (Ena) montanus (Drap.).

Hab. Sur les vieux troncs.

D. h. Gorges de l'Avançon, forêts des Plans et de Pont-deNant.

D. v. Jusqu'à $1500^{\mathrm{m}}$ environ.

\section{Buliminus (Ena) obscurus (Müll.).}

Hab. Sous le bois mort, sur les vieux troncs et parfois sur les rochers.

D. h. Très commun dans toutes les forèts.

D. v. Comme l'espèce précédente.

Gen. Chondrula Beck.

\section{Chondrula quadridens (Müll.).}

Hab. Sous les cailloux et sur des petits murs, dans des champs exposés au soleil.

D. h. Entre les Plans et les Parts.

T. v. De 1100 à $1500^{\mathrm{m}}$ environ.

Fam. Cochlicopidae.

Gen. Cochlicopa Risso.

35. Cochlicopa lubrica (Müll.).

Hab. Sous les écorces, les pierres, la mousse.

D. h. Gorges de l'Avançon, forêts des Plans et de Pont-deNant.

D. v. Jusqu'à $1400^{\mathrm{m}}$ environ. 
Fam. Pupidae.

Gen. Pupa Drap.

36. Pupa (Modicella) avenacea (Brug.).

Hab. Contre les rochers et les vieux murs.

D. h. Tout le long de la route entre Frénières et les Plans; Pont-de-Nant.

D. v. Jusqu'à $1300^{\mathrm{m}}$ environ.

Gen. Vertigo Müll.

37. Vertigo (Alaea) alpestris (Ald.).

Hab. Sur un bloc de pierre moussu.

D. h. Du còté des Parts.

D. v. $1200^{\mathrm{m}}$ environ.

\section{Fam. Clausiliidae.}

Gen. Balea Prid.

38. Balea perversa (L.).

Hab. Sur des troncs et des blocs moussus.

D. h. Frénières, et du còté des Parts.

D. v. Jusqu'à environ $1500^{\mathrm{m}}$.

Gen. Clausilia Drap.

39. Clausilia (Clausiliastra) laminata (Mtg.).

Hab. Sur les vieux troncs, sous les écorces.

D. h. Toutes les forèts; Gorges de l'Avançon, les Plans et Pont-de-Nant.

D. v. Jusqu'à environ $1600^{\mathrm{m}}$. 


\section{Clausilia (Clausiliastra) fimbriata Rossm.}

Hab. Sur les vieux troncs, souvent en compagnie de C. laminala.

D. h. Forêts de Pont-de-Nant et des Plans.

D. v. De 1100 à $1600^{\mathrm{m}}$ environ.

\section{Clausilia (Cusmicia) dubia Drap.}

Hab. Sur les vieux murs et les rochers; sur les vieux troncs, sous les écorces et quelquefois sous les pierres.

D. h. Gorges de l'Avançon; tout le long de la route entre Frénières et les Plans; du côté des Parts; les Plans et Pont-deNant.

D. v. Jusqu'à $1600^{\mathrm{m}}$ environ, au Pont-de-Nant.

\section{Clausilia (Cusmicia) parvula Stud.}

Hab. Sur les vieux murs et les rochers; sous le bois mort, mais plus rarement.

D. h. Entre Frénières et les Plans; chemin de Pont-de-Nant.

D. v. Jusqu’à $1250^{\mathrm{m}}$ environ.

\section{Clausilia (Pirostoma) plicatula Drap.}

Hab. Sous les écorces et les pierres; sur les blocs moussus.

D. h. Forêts des Gorges de l'Avançon, des Plans et de Pontde-Nant.

D. v. Jusqu'à 1500-1600"m environ.

44. Clausilia (Pirostoma) ventricosa Drap.

Hab. Sur de vieux murs et contre les rochers.

D. h. Entre Frénières et les Plans.

D. v. Jusqu'à environ $1200^{\mathrm{m}}$. 


\section{Fam. Succineidae.}

\section{Gen. Succinea Drap.}

\section{Succinea (Amphibina) pfeifferi Rossm.}

Var. recta Baudon.

Hab. Dans de petits ruisseaux et au bord des fontaines de bois.

D. h. Aux environs des Plans.

D. v. $1000-1200^{\mathrm{m}}$.

Remarque: Les individus àgés d'un an sont assez différents de ceux qui ont déjà deux ans, et se rapprochent de la var. contortula que Cuessin mentionne à Bex.

\section{S.-Gl. PNEUMOPOMA}

Fam. Cyclophoridae.

S. - Fam. Pomatiasine.

Gen. Pomatias Stud.

46. Pomatias septemspirale (Razoum.).

Hab. Sous les pierres, sur les rochers, sous le feuillage mort, se confondant toujours, par sa couleur, avec le milieu environnant.

D. h. Gorges de l'Avançon et forêts des Plans.

D. v. Jusqu'à $1300^{\mathrm{m}}$ environ. 


\section{Mollusques récoltés au Val Ferrel (Valais).}

Je cite ici toutes les formes que j'ai trouvées au Val Ferret, surtout aux environs de Praz-de-Fort et de Ville-de-Issert. Prazde-Fort est à une altitude de $1153^{\mathrm{m}}$; on y trouve encore des espèces inférieures, ainsi que quelques formes alpines, qui vivent toutes ensemble. Peu au-dessus de la plaine de Saleinaz, en suivant la combe de la Reuse du còté du glacier de Saleinaz, on trouve à $1300^{\mathrm{m}}$ la limite supérieure des arbres, à laquelle fait suite une région très intéressante d'éboulis et d'anciennes moraines, où croît une petite végétation alpine et où vivent encore un certain nombre de Mollusques d'un assemblage assez curieux : Limax maximus et tenellus, Agriolimax agrestis, Vitrina diaphana, Euconulus fulous, Arion subfuscus, Patula ruderata, Helicodonta holoserica et Campylea foetens. Cette dernière espèce monte très haut, le long du glacier. Mais la limite des arbres, qui est à une faible altitude à cause de la proximité du glacier, atteint $1850^{\mathrm{m}}$ environ au-dessus de Ferret, dans le fond de la vallée, et à peu près $2000^{\mathrm{m}}$ sur les flancs des montagnes.

Autre fait à noter : il existe dans cette partie du Val Ferret, peu au-dessus de Praz-de-Fort, une toute petite forme alpine de l'Eulola fr'uticum qu'on trouve entre 1100 et $1400^{\mathrm{m}}$ environ, c'est-à-dire à une altitude assez faible, puisque la Tachea sylvatica y est encore représentée sous sa forme normale et non pas par sa forme montana. On y trouve encore de gros exemplaires d'Arianta arbuslorum dont la var. alpicola vit à partir d'environ $1500^{\mathrm{m}}$. Enfin la Xerophila obvia, espèce originaire de l'Europe orientale, atteint, à la Seilo, environ $1510^{\mathrm{m}}$. Elle a du reste été signalée dans l'Engadine jusqu'à $1200^{\mathrm{m}}$, mais n'a jamais été mentionnée dans les Alpes occidentales de Suisse.

J'ai trouvé jusqu'à présent 60 espèces au Val Ferret, avec un certain nombre de variétés dont quatre sont nouvelles. 


\section{GASTROPODA}

\section{S.-Cl. PULMONATA}

\section{Ord. STYLOMMATOPHORA}

\section{Fam. Vitrinidae.}

Gen. Limax Müll.

\section{Limax (Heynemannia) maximus L.}

Hab. Sous les pierres, les écorces et le feuillage mort.

D. h. Dans toutes les forêts de la vallée : du còté de Champex, la Deuvaz, Praz-de-Fort, Froumion, anciennes moraines qui bordent la plaine de Saleinaz, au-dessous du glacier de Saleinaz, forêts de la Tête-Moutse, la Neuva, forêts du Jurassa et de Ferret.

D. v. Jusqu'à $1400^{\mathrm{m}}$ sous le glacier de Saleinaz, ailleurs jusqu'à près de $1900^{\mathrm{m}}$.

a) Var. cinerea (List.). On ne rencontre guère cette variété que dans le fond de la vallée jusqu'à Praz-de-Fort.

b) Var. cinereoniger (Wolff). Très répandue partout.

\section{Limax (Heynemannia) tenellus Nils.}

Hab. Sur les vieux troncs, sous les pierres et le feuillage mort.

D. h. Dans les forêts de Praz-de-Fort, des deux côtés de la Reuse (Froumion), forêts du Jurassa, Ferret. Au-dessous du glacier de Saleinaz.

D. v. Jusqu'à près de $1900^{\mathrm{m}}$, comme l'espèce précédente. 
Gen. Agriolimax Simr.

3. Agriolimax agrestis (L.).

Hab. Dans les champs et les jardins potagers; sous les écorces des vieux troncs et les pierres plates; dans les anciennes moraines, sous les blocs granitiques.

D. h. Très commune dans tout le fond de la vallée entre Orsières et Ferret. Répandue dans la plaine de Saleinaz et sur les bords du torrent, jusqu'au-dessous du glacier. Dans les forêts de Froumion et de l'Avary, de la Tête-Moutse, du Jurassa et de Ferret. On trouve dans la plaine de Saleinaz des individus albinos.

D. v. $800-1900^{\mathrm{m}}$. Sous le glacier de Saleinaz, elle n'atteint qu'une altitude bien inférieure, mais la température y est beaucoup plus froide et les conditions d'existence bien plus difficiles; il n'y a plus que très peu de végétation : c'est déjà la région alpine de Ciessin.

\section{Gen. Vitrina Drap.}

4. Vitrina (Phenacolimax) pellucida (Müll.).

Hab. Sur les blocs moussus et sur les vieux troncs.

D. h. Forèts de Froumion et de l'Avary; la Neuva, Ferret.

D. v. Jusqu'à $1850^{\mathrm{m}}$ environ.

\section{Var. alpina Stenz.}

D. h. Tour de Bavon.

D. v. Jusqu'à $2481^{\prime \prime}$.

\section{Vitrina (Semilimax) diaphana Drap.}

Hab. Sous les blocs granitiques moussus, sous les pierres, les écorces, le feuillage mort, sur les vieux troncs. 
D. h. Forèts près de Champex, de Froumion, de l'Avary, Praz-de-Fort; sur les bords de la Dranse, la Neuva, Ferret; tour de Bavon.

D. v. $1150-2481^{\mathrm{m}}$.

\section{Vitrina (Semilimax) nivalis Charp.}

Hab. Assez profondément sous les grosses pierres, au bord de grandes taches de neige; ordinairement isolée, rarement plus de 2 exemplaires à la fois.

D. h. Près de la cabane de Saleinaz, à quelques centaines de mètres du glacier de l'Evole, au-dessus de celui de Saleinaz.

D. v. $2700^{\mathrm{m}}$ environ.

\section{Gen. Hyalina Fér.}

7. Hyalina (Euhyalina) glabra (Stud.).

Hab. Sous le bois pourri et sous les pierres.

D. h. Praz-de-Fort, forèts de l'Avary, la Neuva, Ferret.

D. v. $1150-1850^{\mathrm{m}}$ environ.

\section{Hyalina (Euhyalina) cellaria (Müll.).}

Hab. Sous les pierres et sur des rochers.

D. h. Praz-de-Fort, au bord de la Dranse, au bas de la plaine de Saleinaz.

D. v. Jusqu’à $1160^{\mathrm{m}}$ environ.

9. Hyalina (Euhyalina) depressa Sterki.

Hab. Sous le bois et le feuillage mort.

D. h. Forèts de l'Avary et de la Neuva.

D. v. $1200-1600^{\mathrm{m}}$ environ. 
10. Hyalina (Polita) nitens (Mich.).

Hab. Sous les pierres, les écorces, sur les vieux troncs, les blocs moussus, etc.

D. h. Très commune dans toutes les forèts, Champex, Prazde-Fort, Froumion, l'Avary, Jurassa, la Neuva, flancs de la TèteMoutse, Ferret.

D. v. De 800 à $1850^{\mathrm{m}}$ : régions inférieure et supérieure des forèts; elle ne dépasse guère cette limite.

\section{Var. dutaillyana (Mabille).}

D. h. Dans les mèmes localités que le type, mais plus commune à mesure que l'on s'élève.

D. v. $1200-1850^{m}$.

\section{Hyalina (Polita) pura (Ald.).}

Hab. Sur les blocs moussus, sous les pierres et l'écorce.

D. h. Forèts de Champex; Praz-de-Fort, au bord de la Dranse; forèts de Froumion, de l'Avary, de la Neuva et de Ferret.

D. v. Jusqu'à $1850^{\mathrm{m}}$ environ.

\section{Hyalina (Polita) radiatula Gray.}

Hab. Dans les mèmes conditions que l'espèce précédente.

D. h. Je n'ai trouvé le type qu'à Praz-de-Fort, sur les bords de la Dranse.

D. v. Jusqu'à $1200^{\mathrm{m}}$ environ.

\section{Var. petronella (Charp.).}

D. h. Praz-de-Fort, avec le type; la Neuva et Ferret.

D. v. Jusqu'à $1850^{\mathrm{m}}$ environ, comme la $H$. pura. 
Gen. Crystallus Lowe.

\section{Crystallus crystallinus (Müll.).}

Var. eburnea Htm. (= Hyalina andreaei Böttg.).

Hab. Sur de gros blocs moussus.

D. h. Forêts de la Neuva.

D. v. Jusqu'à $1600^{\mathrm{m}}$ environ.

\section{Fam. Naninidae.}

Gen. Euconulus Reinh.

\section{Euconulus fulvus (Müll.).}

Hab. Sous les pierres, les écorces, la mousse; sur les vieux troncs et les blocs granitiques. Dans les étendues rocailleuses de la région alpine.

D. h. Environs de Champex, de Praz-de-Fort, forêts de Froumion, de l'Avary, du Jurassa, au-dessous du glacier de Saleinaz, forêts de la Fauly, de la Neuva et de Ferret; Tour de Bavon, jusqu'au sommet.

D. v. Jusqu'à $1500^{\mathrm{m}}$ environ au-dessous du glacier de Saleinaz, jusqu'à $1850^{\mathrm{m}}$ dans le fond de la vallée, et elle atteint $2481^{\mathrm{m}}$ à la Tour de Bavon.

Fam. Arionidae.

Gen. Arion Fér.

15. Arion empiricorum Fér.

Hab. Dans les champs ou dans les forêts, sous les pierres, le bois pourri et le feuillage mort.

D. h. Dans le bas de la vallée, jusqu'à Praz-de-Fort et à la plaine de Saleinaz.

D. v. Jusqu'à $1300^{\mathrm{m}}$ environ. 


\section{Arion subfuscus (Drap.).}

Hab. Sous les pierres, le bois pourri, dans les mèmes conditions que l'espèce précédente; parmi les-restes de moraines granitiques; dans les étendues couvertes de Rhododendrons et mème au-dessus de la limite supérieure de ces végétaux.

D. h. Environs de Praz-de-Fort, plaine de Saleinaz, forèts de l'Avary et de Froumion, forèts de la Fauly, de la Neuva, de Ferret. Sur les bords du torrent, au-dessous du glacier de Saleinaz et le long du glacier jusqu'au-dessus des Rhododendrons, en suivant le chemin de la cabane. Forèts de Jurassa, de la Chasse et Tour de Bavon. Tète-Moutse.

D. v. Jusqu'à $2500^{\mathrm{m}}$ environ.

\section{Arion hortensis Fér.}

Hab. Dans les champs ou dans les forèts, sur les vieux trones, sous l'écorce.

D. h. Répandu dans tout le fond de la vallée, jusqu'à Ferret. Sur les flancs de la Tour de Bavon et au-dessus du glacier de Saleinaz, sur le chemin de la cabane.

D. v. Jusqu'à $1850^{\mathrm{m}}$ environ dans le fond de la vallée et jusqu'à environ $2000^{\mathrm{m}}$ à Saleinaz.

\section{Fam. Polyplacognatha.}

\section{Gen. Sphyradium (Charp.) West.}

\section{Sphyradium gredieri (Cless.).}

Hab. Sur des herbes sèches.

D. h. Sommet de la crète qui borde la plaine de Saleinaz, à l'Avary. Rare.

D. v. Environ $1300^{\mathrm{m}}$. 
Gen. Punctum Morse.

\section{Punctum pygmaeum (Drap.).}

Hab. Sur de gros blocs moussus; sous les feuilles mortes.

D. h. Forêts de Froumion et de l'Avary; la Fauly et la Neuva. Tour de Bavon jusqu'au sommet.

D. v. Jusqu'à $2481^{\mathrm{m}}$.

\section{Fam. Patulidae.}

Gen. Patula Heid.

\section{Patula (Discus) rotundata (Müll.).}

Hab. Sous le bois mort et les pierres.

D. h. Très commune dans le bas de la vallée: Orsières, Somla-Proz, Ville-d'Issert, Praz-de-Fort, la Deuvaz, les Arlaches. Encore assez commune à la Neuva, mais rare à Ferret.

D. v. $1400^{\mathrm{m}}$ à la Deuvaz, $1600^{\mathrm{m}}$ à la Neuva et $1800^{\mathrm{m}}$ à Ferret, ce qui fait élever de $200^{\mathrm{m}}$ la limite supérieure que Bollinger assigne à cette espèce (Chamony : $\left.1600^{\mathrm{m}}\right)^{1}$.

\section{Patula (Discus) ruderata (Stud.).}

Hab. Dans les mêmes conditions que l'espèce précédente. La var. viridana vit sur les blocs moussus, le type plus rarement.

D. h. Dans tout le haut de la vallée, depuis Praz-de-Fort. TêteMoutse et flancs de la Tour de Bavon.

D. v. Jusqu'à $2000^{\mathrm{m}}$ environ; $1500^{\mathrm{m}}$ au-dessous du glacier de Saleinaz.

\section{Var. viridana Roff.}

D. h. Cette variété, plus mince que le type, verdâtre, très fragile, semble provoquée par une espèce de Lichen, qui offre

${ }^{1}$ Lehmann (1911) dit même avoir trouvé cette espèce jusqu'à 1970 m, au Sigriswylgrat. 
souvent une belle coloration vert clair : forèts de l'Avary et de la Neuva.

D. v. $1200-1600^{\mathrm{m}}$ environ.

Gen. Pyramidula Fitz.

\section{Pyramidula rupestris (Drap.).}

Hab. Contre les rochers. Dans les étendues rocailleuses de la région alpine.

D. h. Le long de la route d'Orsières à Praz-de-Fort; au pied de la Tète-Moutse, sur les bords de la Dranse; sur des rochers avant d'arriver à Ferret et dans la forêt qui fait suite à ce village. Tour de Bavon, jusqu'au sommet.

D. v. Jusqu'à $1850^{\mathrm{m}}$ après Ferret et $2481^{\mathrm{m}}$ à la Tour de Bavon.

\section{Fam. Eulotidae.}

Gen. Eulota Htm.

\section{Eulota fruticum (Müll.).}

Hab. Dans les champs, au pied des haies et des buissons. La var. godetiana dans des pâturages.

D. h. Le type de cette espèce ne s'avance guère que jusqu'à la plaine de Saleinaz. La Deuvaz. (Fig. 4.)

D. v. Le type monte jusqu'à environ $1200^{\mathrm{m}}$, dans le fond de la vallée, mais il atteint $1400^{m}$ à la Deuvaz, sur le versant exposé au soleil.

\section{Var. godetiana Piaget.}

D. h. Au-dessous de Praz-de-Fort et jusque bien en amont de ce village, dans le fond de la vallée.

D. v. De 1100 à $1400^{\mathrm{m}}$ environ.

Cette variété est remarquable par sa petite taille; on trouve 
aux environs de Praz-de-Fort une foule d'intermédiaires entre elle et le type de l'espèce. M. de Monterosato m'a fait remarquer que ma variété rappelle la var. insulorum de Westerlunv.

F. normalis. $12-15^{\mathrm{mm}}$ de diamètre, $10-11^{\mathrm{mm}}$ de hauteur. (Fig. $5-8$.

F. conoidea, f. depressa, etc.

F. intermedia. $15-17^{\mathrm{mm}}$ de diamètre; plaine de Saleinaz, etc. (Fig. 5-6.)

Mut. luteola, rubella, roseo labiala.

\section{Fam. Helicidae.}

S.-Fam. Valloninae.

Gen. Vallonia Risso.

24. Vallonia pulchella (Müll.).

Var. helvetica Sterki.

Hab. Dans les champs sous les pierres; sur les rochers.

D. h. Au bas de la plaine de Saleinaz, sur des blocs de rocher au bord de la Dranse. La Deuvaz.

D. v. Jusqu'à $1420^{\mathrm{m}}$ environ.

S.-Fam. Helicodontinae.

Gen. Helicodonta Risso.

25. Helicodonta (Trigonostoma) obvoluta (Müll.).

Hab. Sous les pierres, le bois mort, les écorces.

D. h. Du còté de Champex; forèts de Froumion et de l'Avary.

D. v. Jusqu'à $1300^{\mathrm{m}}$ environ. Cette espèce monte beaucoup moins haut que la Patula rolundata, avec laquelle elle vit ordinairement; le mème fait se reproduit dans d'autres vallées. 


\section{Helicodonta (Trigonostoma) holoserica (Stud.).}

Hab. Sous les pierres, les anciennes moraines, ou plus rarement sur les vieux troncs.

D. h. Trouvée par M. Paul Govet sur les bords du lac Champex. Forèts de Froumion et de l'Avary, au-dessus de Praz-de-Fort. Anciennes moraines de la plaine de Saleinaz, jusqu'assez haul, dans la direction du glacier. Forèts de la Neuva et de Ferret; flanes de la Tour de Bavon.

D. v. Jusqu'à $2000^{\mathrm{m}}$ environ.

Aux environs immédiats de Praz-de-Fort, cette espèce vit très souvent dans les mèmes conditions que la Campylaea foetens.

\section{S.-Fam. Fruticicolinae.}

Gen. Fruticicola Held.

\section{Fruticicola (Perforatella) edentula (Drap.).}

Hab. Sur les vieux troncs et sous les pierres.

D. h. Forèts de Champex, de Froumion et de l'Avary audessus de Praz-de-Fort, la Fauly et la Neuva.

D. v. Jusqu'à $1600^{\mathrm{m}}$ environ.

\section{Fruticicola (Fruticicola s. str.) sericea (Drap.).}

Hab. Sur les vieux troncs et sous les pierres, dans les forêts ; souvent aussi au bord des routes, sur les rochers humides. La var. corneola dans les champs secs.

D. h. Très commune dans le fond de la vallée d'Orsières à Ferret; forèts de Champex. Tour de Bavon, jusqu'au sommet (exemplaires normaux, vivant sous les pierres).

D. v. Jusqu'à $1850^{\mathrm{m}}$, au fond de la vallée, jusqu'à $2481^{\mathrm{m}}$ à la Tour de Bavon. Bollinger ne cite pas cette espèce à plus de $2000^{\text {mit }}$ (Urden Alp.). 
29. Fruticicola (Euomphalia) strigella (Drap.).

Hab. A la lisière des forèts, sous les pierres et le feuillage mort.

D. h. Plaine de Saleinaz.

D. v. Environ $1150-1300^{\mathrm{m}}$.

\author{
S.-Fam. Cimpilaeinae.
}

Gen. Campylaea Beck.

30. Campylaea foetens (Stud.).

Hab. Sous de gros blocs granitiques et moussus, souvent à quelques mètres de la surface de la moraine. Sous de grosses pierres, au bord de grandes taches de neige, en compagnie de Vitrina nisalis.

D. h. Dans des moraines assez récentes et couvertes de mousse, des deux còtés de la plaine de Saleinaz. Dans une vieille moraine couverte de forêts, de l'autre còté de la Reuse à Froumion; dans des conditions semblables à Prayon et à la Seilo. Au milieu de blocs charriés par la rivière, jusque sous le glacier de Saleinaz et dans les mêmes conditions à la Neuva.

" Une forme plus petite existe sur le M' Catogne mème, vers le milieu de la montée, sur des blocs entassés dans un fond, endroit très caractéristique. La mème var. a été trouvée par M. Kunz, l'alpiniste bien connu, à $2200^{\mathrm{m}}$ au Val Ferret, dans le voisinage du glacier de Saleinaz " (Godet, manuscr.).

J'ai trouvé cette même forme aux environs de la cabane de Saleinaz.

D. v. A partir de $1170^{\mathrm{m}}$ jusqu'à environ $2700^{\mathrm{m}}$.

Remarque: M. le Dr Paul Godet m'a donné autrefois des exemplaires de cette espèce, sous le nom d'Helix foetens Stud., provenant les uns de Saleinaz, les autres des pentes du Catogne, 
où l'indique Charpenter (p. 8). Contrairement aux avis de Chessin et de Charpentien, l'opinion de M. Godet était que l'H. foetens de Studer n'est ni un synonyme mal défini de la Compylaea ichthyomma, qui est bien différente, ni une variété de l'H. zonala Studer, "dont elle se distingue non seulement par un test plus brillant et par l'odeur musquée plus forte que répand l'animal, mais par une spire plus déprimée et par la forme de l'ouverture beaucoup moins arrondie, etc. " Godet, in sched.

Gen. Chilotrema Leach.

\section{Chilotrema lapicida (L.).}

Hab. Sous les pierres et le bois mort; sur les rochers.

D. h. Dans le bas de la vallée : environs d'Orsières et de Somla-Proz; Ville-d'Issert et le long du chemin de Champex, jusqu'aux forêts. La Deuvaz; environs de Praz-de-Fort, dans les forêts de Froumion, de l'Avary et dans la plaine de Saleinaz.

D. v. Elle atteint $1400^{\mathrm{m}}$, soit à la Deuvaz, soit dans les forêts de Praz-de-Fort.

Gen. Arianta Leach.

32. Arianta arbustorum (L.).

Hab. Dans les champs et les forèts.

D. h. Le type est répandu dans le bas de la vallée: Orsières, Som-la-Proz, Ville-d'Issert, chemin de Champex, la Deuvaz, Praz-de-Fort, jusque dans la plaine de Saleinaz.

D. v. Le type monte jusqu'à $1300^{\mathrm{m}}$ environ dans le fond de la vallée, mais il atteint $1400^{\mathrm{m}}$ à la Deuvaz.

Remarque : Les exemplaires de cette partie de la vallée sont intermédiaires entre la forme alpine et la forme de la plaine, qui est le type de l'espèce, mais ils se rapprochent plus du type que de la variété. Ils sont identiques à ceux des Plans, de 
Neuvaz, etc., bien plus grands que ceux des Haudères, de la Gemmi, de Kandersteg, etc., et bien plus petits que ceux du Plateau (Cerlier), du Jura inférieur (environs de Neuchâtel, etc.) et de la Franche-Comté (Pont-de-Roide, Audincourt, etc.).

\section{Var. alpicola Charp.}

Hab. Dans les champs, les forèts et les alpages supérieurs.

D. h. Partie supérieure de la vallée, au-dessus de Praz-deFort, Prayon, la Seilo, l'Amòna, la Neuva, la Fouly, environs de Ferret. Tète Moutse et Tour de Bavon.

D. v. Jusqu'à $1850^{\mathrm{m}}$ environ au fond de la vallée, $2100^{\mathrm{m}}$ à la Tète Moutse et $2481^{\mathrm{m}}$ à la Tour de Bavon. On la retrouve à partir de 1200-1500 ${ }^{\mathrm{m}}$ environ.

$\mathrm{M}^{\mathrm{Hle}} \mathrm{A}$. Dubied a recueilli à Praz-de-Fort un monstre scalaire appartenant à cette variété.

\section{Gen. Isognomostoma Fitz.}

\section{Isognomostoma personatum (Lam.).}

Hab. Sous les pierres, le bois et le feuillage mort; sur les vieux trones.

D. h. Forèts de Froumion, de l'Avary et de la Neuva.

D. v. $1150-1600^{\mathrm{m}}$ environ.

S.-Fam. Helicinae.

Gen. Helix L., s. str.

34. Helix pomatia L.

Hab. Dans les champs et à la lisière des forèts; la var. parva dans les forèts.

D. h. Assez commune dans le fond de la vallée: Orsières, Som-la-Proz, Ville-d'Issert, chemin de Champex, la Deuvaz, Praz-de-Fort; à partir de là, moins commune, jusqu'à Ferret. 
D. v. Jusqu'à $1800^{\text {m }}$ environ. C'est la limite supérieure que Bollinger assigne à cette espèce.

F. normalis (var. rustica Atm.) Commune partout, avec des variations de couleur: mut.trifasciala, quadrifasciata, quinquefasciata, efasciata.

F. plus minusve elevata. Surtout du còté de Ferret.

\section{Var. parva Moq. Tand.}

D. h. J'en ai trouvé un bel exemplaire dans la forêt, près de la Deuvaz: $30^{\mathrm{mm}}$ de hauteur et 30 de diamètre. Le péristome légèrement évasé et de couleur rose indique bien que l'individu est adulte.

D. v. A $1400^{\mathrm{m}}$ environ.

\section{Var. pulskyana Hazay.}

D. h. Plaine de Saleinaz.

D. v. $1150-1300^{\mathrm{m}}$ environ.

J'ai trouvé de nombreux exemplaires un peu jeunes de cette jolie petite variété, de forme élevée et à cinq fascies ininterrompues.

\section{Gen. Tachea Leach.}

\section{Tachea sylvatica (Drap.).}

Hab. Je n'ai trouvé le type de l'espèce que contre des rochers. La var. montana vit aussi dans les forèts.

D. h. Le long de la route entre Orsières et Praz-de-Fort et un peu au-dessus de ce village.

D. v. Jusqu'à environ $1300^{\mathrm{m}}$.

\section{Var. montana Stud.}

D. h. Au-dessus de Praz-de-Fort, dans les forêts de Froumion, de l'Avary, des flancs de la Tète Moutse et du Jurassa. Dans le fond de la vallée jusqu'à Ferret. Tour de Bavon, jusqu'au 
sommet où j'ai trouvé une toute petite forme blanchâtre, très peu ponctuée de brun très clair, à péristome rosé; elle est en outre très déprimée.

D. v. De $1300-1400^{\mathrm{m}}$ à $2481^{\mathrm{m}}$. Bollunger la cite à $1800^{\mathrm{m}}$ au Val Ferret et lui donne comme limite supérieure $2300^{m}$ à la Gemmi.

On trouve fréquemment des exemplaires albinos Helix aimophilopsis Bourg.) appartenant surlout au type (var. C. albinos Charp., 1837 , p. 6).

S.-Fam. Xerophulinae.

Gen. Xerophila Held.

36. Xerophila (Xerophila s. str.) ericetorum (Mull.).

Hab. Dans les champs très secs, bien exposés au soleil.

D. h. Environs d'Orsières, de Som-la-Proz et de Ville d'Issert, chemin de Champex; magnifiques exemplaires à la Deuvaz. Prazde-Fort et dans le fond de la vallée, jusqu'à la Seilo et à la Fauly.

D. v. Jusqu'à $1550^{\mathrm{m}}$ dans le fond de la vallée et jusqu'à $1400^{\mathrm{m}}$ sur le versant ouest, à la Deuvaz.

\section{Xerophila (Xerophila) obvia (Htm.).}

Hab. Dans la partie inférieure de la vallée, elle habite les champs de céréales très exposés au soleil, sur les tiges des plantes et les murs de clòture; plus haut, on la trouve dans des prairies plus humides et au bord des chemins.

D. h. En 1911, je ne l'ai trouvée que d'Orsières à Som-la-Proz, en grande abondance tout le long de la route. Elle ne devait pas s'y trouver en 1909, ou du moins y ètre assez rare, car M. Godet, que la question de l'invasion des Xerophila obvia a particulièrement intéressé, ne l'y a pas trouvée, bien qu'il ait fait ce trajet deux fois pendant cette année. De 1911 à 1912, cette espèce s'est répandue bien au delà de Som-la-Proz, presque jusqu'à Ville-d'Issert, sur un parcours que j'avais bien étudié à ce sujet 
en 1911 et sur lequel je n'avais pas vu trace de $X$. obvia, alors qu'elle y est déjà maintenant très commune. En outre, deux nouvelles colonies sont parties de ce nouveau centre pour se fixer, la première, peu nombreuse, à la Deuvaz, au-dessus de Praz-de-Fort, en compagnie de X. ericetorum, et la seconde, plus nombreuse, à la Seilo sur un espace de quelques mètres, au bord de la route, également en compagnie de X. ericetorum. Il sera très intéressant de voir, dans quelques années, jusqu'où sera allé le rayonnement de cette dernière colonie, à une altitude tout à fait insolite pour cette espèce orientale.

D. v. En 1911 elle ne s'avançait pas au-dessus de $973^{m}$. Le nouveau centre de la Deuvaz est à $1400^{\mathrm{m}}$ environ. Sa marche lente d'Orsières au fond de la vallée arrive actuellement à peu près à 1000-1025 , entre Som-la-Proz et Ville-d'Issert, tandis qu'à la Seilo elle s'étend entre 1500-1510m environ.

D. en Suisse. Cette espèce n'est connue, en Suisse, que depuis peu de temps; Chessin, en 1887, ne l'y mentionne pas encore. Monterosato l'a trouvée à Rorschach, dans le canton de SaintGall; Schenk au Ramsen; Zschoкке dans la vallée de l'Albula (Tiefenkastel); Carl l'a rapportée de l'Engadine, où elle vit jusqu'à $1200^{\mathrm{m}}$. On la trouve à Schuls et à Tarasp. Bollinger l'a recueillie à divers endroits, près de Bàle; Godet, en 1907, ne la signale qu'au Chanet, sur Neuchâtel. Je l'ai recueillie depuis dans différentes localités du canton de Berne occidental et de la région s'étendant entre les lacs de Neuchâtel et de Bienne Pont de Thielle, la Sauge, Bethléhem, Cerlier, route du Landeron à Lignières), ainsi que jusqu'à Peseux, depuis le Chanet. Mon ami Reichel m'en a rapporté de Lausanne et de la grève de Vidy; je l'ai également trouvée aux environs de Prangins. Kampuans la cite près d'Annemasse.

Dernièrement M. le Prof. O. Stroll m'écrivait: "La X. obvia est dans ma collection des environs de Sion...; depuis une dizaine d'années, je l'ai constatée près d'Affoltern canton de Zurich), dans un seul endroit, il est vrai, mais j'en ai ramassé plus de 150 échantillons sans en diminuer visiblement le nombre; elle reparait chaque année. ” En janvier 1913, M. Meylan, 
de Sainte-Croix, le phytologiste bien connu, me communiquait qu'en cherchant des Mousses, au Valais, il venait de trouver la Xerophila obvia sur Branson (près Martigny).

On ne l'avait cependant jamais signalée dans les Alpes occidentales, et j'en ai récolté un nombre considérable : tout le long de la roule entre Sembrancher et Orsières et entre Sembrancher et Châbles; à Isérables; aux environs de Sion (chemin d'Aproz, route des Agettes, de Salins et Baar) et à Euseigne (voir plus loin).

38. Xerophila (Striatella) candidula (Stud.).

Hab. Dans les champs, avec les autres espèces de ce genre.

D. h. Environs de Praz-de-Fort, la Deuvaz, plaine de Saleinaz.

D. v. Jusqu'à $1400^{\mathrm{m}}$ environ.

$$
\text { Var. gratiosa (Stud.). }
$$

D. h. Praz-de-Fort, sur la route de Ville-d'Issert et sur le chemin de la Deuvaz.

D. v. Jusqu'à $1200^{\mathrm{m}}$ environ.

Fam. Buliminidae.

Gen. Buliminus Ehr.

\section{Buliminus (Zebrina) detritus (Müll.).}

Hab. Dans les champs de céréales, avec les espèces précédentes.

D. h. Environs d'Orsières, sur la route de Ville-d'Issert, chemin de Champex, la Deuvaz, environs de Praz-de-Fort et au bas de la plaine de Saleinaz.

D. v. Jusqu'à $1400^{\mathrm{m}}$ environ. 
40. Buliminus (Ena) montanus (Drap.).

Hab. Sur les vieux troncs et sous le feuillage mort.

D. h. Forèts de Froumion et de l'Avary, la Deuvaz, environs de Champex, la Neuva et Ferret.

D. v. Jusqu'à $1800^{\mathrm{m}}$.

\section{Buliminus (Ena) obscurus (Müll.).}

Hab. Sous les pierres, sur les rochers et les vieux troncs.

D. h. Comme l'espèce précédente et sur les rochers de la route entre Orsières et Praz-de-Fort.

D. v. Jusqu'à $1800^{\mathrm{m}}$. Suivant Bollinger, on ne l'aurait pas encore trouvée en Suisse à cette altitude.

Gen. Chondrula Beck.

\section{Chondrula quadridens (Müll.).}

Hab. Dans les champs de céréales, avec des Xerophila.

D. h. Environs de Ville-d'Issert, la Deuvaz et au bas de la plaine de Saleinaz.

D. v. Jusqu'à environ $1400^{\mathrm{m}}$.

Fam. Cochlicopidae.

Gen. Cochlicopa Risso.

\section{Cochlicopa lubrica (Müll.).}

Hab. Dans les forêts sous les pierres et le feuillage mort; dans les champs et les rocailles.

D. h. Forèts de l'Avary et de la Fauly.

D. v. Jusqu'à $1600^{m}$. 


\section{Var. minima Siemaschko.}

D. h. Dans la plaine de Saleinaz et Tour de Bavon.

D. v. $1150-2481^{\mathrm{m}}$.

\section{Fam. Pupidae.}

Gen. Pupa Drap. ${ }^{1}$

\section{Pupa (Torquilla) secale Drap.}

Hab. Sur les vieux troncs et sous les pierres et le bois mort; dans les étendues herbeuses et rocailleuses de la région alpine (var. minor).

D. h. Environs d'Orsières, Som-la-Proz, Ville-d'Issert, la Deuvaz, Champex, environs de Praz-de-Fort, dans les forèts du Jurassa, de Froumion et de l'Avary, au pied de la Tète-Moutse, Prayon, la Seilo, l'Amòna, la Fouly, environs de la Neuva et de Ferret.

D. v. Jusqu'à $1850^{\mathrm{m}}$.

\section{Var. minor Kregl.}

D. h. Tour de Bavon.

D. v. De 2450 à $2481^{\mathrm{m}}$. (Coquilles de 4, 5 à $6^{\mathrm{mm}}$ de hauteur.)

\section{Pupa (Modicella) avenacea (Brug.).}

Hab. Sur les rochers.

D. h. Environs d'Orsières, de Ville-d'Issert, de Praz-de-Fort et de Ferret.

D. v. Jusqu'à $1850^{\mathrm{m}}$. Elle a atteint cette grande altitude à Alpstein.

1 РАуот, dans son Erpétologie, malacologie et paléontologie des environs du Mont-Blanc (Lyon, 1864), signale (p. 46) le Pupa frumentum Drap., à Orsières; mais il ne doit pas l'avoir rencontrée dans le Val Ferret, car il assigne à cette espèce la limite supérieure de $800^{\mathrm{m}}$, alors qu'Orsières est à $885 \mathrm{~m}$. M. Th. Studer (Lehmans, 1911, p. 103) a trouvé ce même Pupa à Champex (rochers à environ $1600^{\mathrm{m}}$ ) en compagnie de Xerophila ericetorum et Chilotrema lapicida. 
Gen. Pupilla Pfr.

\section{Pupilla muscorum (L.).}

Hab. Sur des rochers ou dans les champs, au bord des chemins.

D. h. La Deuvaz et sur le chemin de Champex, au-dessus de Ville-d'Issert.

D. v. Jusqu'à $1400^{\mathrm{m}}$.

Var. pratensis Cless.

D. h. Plaine de Saleinaz.

D. v. Jusqu'à $1200^{\mathrm{m}}$ environ.

$$
\begin{aligned}
& \text { 47. Pupilla alpicola (Charp.). } \\
& \text { Syn. : P. madida Gredl. }
\end{aligned}
$$

Hab. Sur les rochers herbeux, sous les rocailles.

D. h. Tour de Bavon.

D. v. $2450-2481^{\mathrm{m}}$.

\section{Gen. Vertigo Müll.}

48. Vertigo (Alaea) pygmaea Drap.

Hab. Sur des blocs moussus.

D. h. Forèts de Praz-de-Fort.

D. v. Jusqu'à $1300^{\mathrm{m}}$ environ.

\section{Vertigo (Alaea) alpestris (Ald.).}

Hab. Sur de gros blocs moussus.

D. h. Forèts de Froumion, de l'Avary, de la Neuva et de Ferret.

D. v. $1150-1850^{\mathrm{m}}$. 


\title{
Fam. Clausiliidae.
}

\author{
Gen. Balea Prid.
}

50. Balea perversa (L.).

Hab. Sur des blocs granitiques moussus.

D. h. Forêts de Froumion, de l'Avary, du Jurassa, de la Neuva et de Ferret; entre Som-la-Proz et Ville-d'Issert; Champex. Orsières. (РАуот, 1864, p. 48. .)

D. v. $1000-1850^{\mathrm{m}}$.

Var. vitrina nov, var.

Varietas minor, fragillima, diaphana, viridula; peristoma paene simplex, anguslissime reflexiusculum, interruptum; lamella superior saepius deficit.

Alt. $7-8^{\mathrm{mm}} ;$ diam. 1,05-2 ${ }^{\mathrm{mm}}$.

L'animal est complètement vert, plus ou moins foncé, parfois même assez clair.

Coquille sénestre, de forme allongée très acuminée, très régulière, de plus petite taille que le type, très mince et fragile, complètement transparente, très finement et régulièrement striée, assez brillante, de couleur verdâtre légèrement vitreuse. 8-10 tours de spire, ordinairement 9, s'accroissant lentement, assez convexes, séparés par une suture assez profonde, le dernier tour plus régulier que chez le type, bien arrondi à sa base. Ouverture plus arrondie, entourée d'un très mince bourrelet interrompu. La lamelle pariétale est toujours très petite et très mince, mais le plus souvent elle fait défaut. C'est l'équivalent chez la B. perversa de la var. petronella chez la Hyalina radiatula et de la var. viridana chez la P. ruderata. Elle vit, du reste, en compagnie de cette dernière variété.

M. de Monterosato m'écrit au sujet de cette nouvelle Balea: “Elle rappelle certaines variétés claires, presque sans stries, 
venant du Pouliguen (Còtes océaniques de France). La Balea heydeni de von Maltzan (Journ. Conchyl., 1881, p. 162, pl. VI, f. 6), de Cintra (Portugal), est peut-ètre la mème que celle du Pouliguen. Ce serait aussi la B. lucifuga. »

Hab. Sur de gros blocs moussus couverts de certains Lichens de couleur verte que M. Meycan, de Sainte-Croix, a eu la bonté de me déterminer. Ce sont des Peltigera, soit Peltigera horizontalis Ach., soit des $P$. canina ou encore $P$. rufescens, toutes fréquentes tant sur les blocs que sur les vieux troncs.

D. h. Forèts de l'Avary, au bord de la plaine de Saleinaz.

D. v. $1200-1300^{\mathrm{m}}$.

Gen. Clausilia Drap.

51. Clausilia (Clausiliastra) laminata (Mtg.).

Hab. Sur les vieux troncs, les blocs moussus et sous le bois mort.

D. h. Forèts de Champex, de Froumion, de l'Avary et de la Neuva.

D. v. $1150-1600^{\mathrm{m}}$.

\section{Clausilia (Cusmicia) parvula Stud.}

Hab. Sur des rochers schisteux.

D. h. Au-dessous de la Deuvaz sur le chemin de Champex : près de l'Ardoisière.

D. v. Environ $1350^{\mathrm{m}}$.

Remarque. Cette espèce parait ne vivre que sur les terrains calcaires et très rarement sur les rochers schisteux; je ne l'ai jamais trouvée sur des terrains granitiques. Au val Ferret, je n'en ai vu qu'un bon exemplaire, adulte et vivant.

\section{Clausilia (Cusmicia) cruciata Stud.}

Var. alpestris Stoll.

Hab. Sur les gros blocs moussus, les vieux troncs, sous le bois mort. 
D. h. Forêts de l'Avary et de la Neuva.

D. v. $1200-1600^{\mathrm{m}}$.

\section{Clausilia (Cusmicia) dubia Drap.}

Hab. Sur les vieux troncs ou de gros blocs granitiques, sous la mousse.

D. h. Forêts de Froumion, de l'Avary, du Jurassa, de la Neuva et de Ferret.

D. v. Jusqu'à $1850^{\mathrm{m}}$.

\section{Clausilia (Pirostoma) plicatula Drap.}

Hab. Sur les vieux troncs.

D. h. Dans la forêt du Jurassa, au-dessus de Praz-de-Fort.

D. v. A environ $1300^{\mathrm{m}}$.

\section{Clausilia (Pirostoma) ventricosa Drap.}

Hab. Sur des rochers humides, au bord des routes, ou dans les forêts, sur les vieux troncs et sous le bois mort.

D. h. Environs de Praz-de-Fort, au pied de la Tête-Moutse, forêts du Jurassa et à la Neuva.

D. v. Jusqu'à environ $1600^{\mathrm{m}}$.

\section{Fam. Succineidae.}

\section{Gen. Succinea Drap.}

\section{Succinea pfeifferi Rossm.}

Hab. J'ai trouvé le type sur des rochers humides et la var. contortula au bord des mares et ruisseaux.

D. h. Entre Ville-d'Issert et Praz-de-Fort.

D. v. Jusqu'à $1200^{\mathrm{m}}$ environ. 
Var. contortula Baudon.

D. h. Mares de Froumion.

D. v. $1150-1200^{\mathrm{m}}$ environ.

\section{Ord. BASOMMATOPHORA}

\section{Fam. Limnaeidae.}

Gen. Limnaea Lam.

58. Limnaea (Gulnaria) limosa (L.), sensu latissimo.

$$
\text { Subsp. peregra (Müll.). }
$$

Hab. Dans des mares et dans un petit lac.

D. h. Je n'ai trouvé la forme normale que rarement à Praz-deFort; lac Champex ${ }^{1}$.

D. v. $1150-1472^{\mathrm{m}}$.

a) Var. marginata (Mich.).

Hab. Dans des mares et des petits ruisseaux.

D. h. Froumion, au-dessus de Praz-de-Fort.

D. v. $1180^{\mathrm{m}}$ environ.

b) Var. reicheliana nov. var.

Testa minor (quam typus subsp. "peregra») solida, cinerea, punctis fuscis maculata; spira parum elongala, apertura normalis, intus cornea cum iisdem fuscis punctis; peristoma simplex, acutum.

Alt. $12-16^{\mathrm{mm}} ;$ diam. $7-9^{\mathrm{mm}}$

Apert. $8-9^{\mathrm{mm}} ;$ alta, $4-5^{\mathrm{mm}} \cdot$ lata.

${ }^{1}$ On trouve également au lac Champex la Limnaea auricularia, comme me l'a communiqué M. le Prof. Stolt, ainsi qu'une grosse variété de peregra appelée par Mostenosato L. purpurea, et qui sera prochainement décrite. Celte dernière forme habite également Chamonix et peut atteindre une taille considérable. 
Hab. Dans l'eau courante très froide $\left(4\right.$ à $\left.8^{\circ}\right)$.

D. h. Sur les bords de la Dranse, au-dessus de Praz-de-Fort.

D. v. $1170-1200^{\mathrm{m}}$ environ.

Cette nouvelle variété, que je dédie à mon ami M. Reichel, a un test de grandeur moyenne, solide, assez finement et irrégulièrement strié, parfois légèrement martelé, opaque, gris très cendré, tirant mème sur le blanchâtre ou par places le bleuâtre, ponctué, surtout au dernier tour de spire, de petites taches brunes, caractère que présentent tous les individus adultes; spire intacte, plus ou moins recouverte de dépòts limoneux; forme générale très peu allongée, ovale, plus ou moins ramassée. 4-5 tours de spire peu convexes, s'accroissant assez rapidement, formant une spire conique, un peu acuminée, peu allongée; le dernier tour régulier, non dilaté. Suture très peu profonde. Ouverture ovale, arrondie, occupant les $9 / 13,9 / 14 \mathrm{Ou}^{2 / 3}$ de la longuear totale, brun corné à l'intérieur, avec les mèmes taches brun clair qui se trouvent chacune dans une très petite dépression; l'angle formé au point d'insertion du bord droit est un peu aigu. Columelle tordue, oblique, large, surtout à sa partie supérieure; fente ombilicale assez apparente. Péristome régulier, tranchant, toujours simple.

Par sa coloration, elle est voisine de la var. frigida Charp., variété bien différente par sa forme élancée, sa haute spire et sa petite ouverture. La var. firgidissima Monts., également voisine, diffère de la $L$. reicheliana par sa taille plus petite, sa coloration plus cornée et moins gris cendré, ses points brunàtres moins abondants, son bord extérieur de l'ouverture solide et bordé en dedans au lieu d’ètre mince et simple; enfin, le bord columellaire est beaucoup moins tordu et blanchâtre alors que la $L$. reicheliana l'a assez tordu, plus dilaté à sa partie supérieure et de couleur rose violacé.

\section{c) Var. blauneri Schuttl.}

Hab. Dans une mare.

D. h. Froumion, mare $n^{0} 3$.

D. v. Environ $1160^{\mathrm{m}}$. 
Remarque. Cette variété se distingue des deux suivantes par sa taille moyenne, son test très mince, pâle, et sa grosse ouverture.

\section{d) Var. dautzenbergiana Piaget.}

Hab. Dans de toutes petites mares, très vaseuses.

D. h. Froumion, mares 1 et 2 .

D. v. Environ $1170^{\mathrm{m}}$.

Remarque. Cette variété se distingue de la suivante par son test un peu plus solide, plus petit, plus foncé (rouge brun), plus ramassé, ouverture plus arrondie.

La var. Dautzenbergiana (PIAget, Journ. Conch., 1911, LIX, p. 339 ) a une coquille très petite, assez fragile, très finement et régulièrement striée, un peu transparente, cornée, brun rougeâtre, plus ou moins érodée, à spire presque toujours tronquée, de forme générale assez ramassée, ovale-allongée. 4 tours de spire assez convexes, s'accroissant assez rapidement, formant une spire peu allongée, obtuse, légèrement conique, mais le plus souvent ${ }^{95}$ ion) fortement attaquée, les 2 premiers tours tronqués, le dernier régulier, non dilaté; suture peu profonde. Ouverture ovale arrondie, occupant les ${ }^{2} / 3$ de la longueur totale; l'angle formé au point d'insertion du bord droit est assez aigu. Columelle presque droite, très mince, à peine dilatée à sa partie supérieure; fente ombilicale plus ou moins ouverte, mais toujours perceptible. Péristome extrèmement mince, simple, tranchant.

Hauteur sans la spire $7-8^{\mathrm{mm}}$, rarement 9 ; largeur $4-5^{\mathrm{mm}}$. Ouverture haute de $4-5^{\mathrm{mm}}$, large de $3-4^{\mathrm{mm}}$.

$$
\text { e) Var. corrosa Dum. et Mort. }
$$

Hab. Mare et ruisseaux.

D. h. Froumion.

D. v. $1160-1170^{\mathrm{m}}$ environ.

59. Limnaea (Fossaria) truncatula (Müll.).

Hab. Dans des mares ou sur des rochers humides. 
D. h. Orsières, Ville-d'Issert, Praz-de-Fort, Froumion, Champex et Bavon.

D. v. Jusqu'à $1300^{\mathrm{m}}$ au-dessus de Praz-de-Fort, $1500^{\mathrm{m}}$ à Champex, $2200^{\mathrm{m}}$ à Bavon.

\section{ACEPHALA}

Fam. Sphaeriidae.

Gen. Pisidium C. Pfr.

60. Pisidium (Fossarina) fossarium Cl.

Hab. Enfouie dans la vase à quelques centimètres de profondeur, dans des mares, des ruisseaux et des rivières.

D. h. Mares de Froumion, dans la Dranse au-dessus de Prazde-Fort, marais de Champex; Bavon.

D. v. Jusqu'à $1300^{\mathrm{m}}$, au-dessus de Praz-de-Fort, $1500^{\mathrm{m}}$ à Champex et $2200^{\mathrm{m}}$ à Bavon.

\section{Var. major Gredl.}

D. h. Mares 1 et 2 de Froumion; Bavon.

D. v. $1170-2200^{\mathrm{m}}$.

Mollusques récoltés au Val-de-Nendaz (Valais).

Quoique le Val-de-Nendaz ne s'avance pas beaucoup au sud, comme le Val-d'Hérens par exemple, il présente cependant, sur une petite surface, un grand nombre de régions différentes peuplées chacune par les espèces caractéristiques. C'est ainsi que la Dent-de-Nendaz, située sur le bord même de la vallée 
du Rhòne, est habitée, près de son sommet, par la Vitrina nivalis, dans les forêts supérieures par les espèces alpines habituelles (Helicodonta holoserica, etc.), plus bas par les espèces sylvatiques des régions inférieures (Hel. obvoluta, etc.), et enfin, à son pied, par la faune caractéristique du bas des vallées.

C'est sans doute précisément à cause de l'accumulation des régions hypsométriques superposées sur une toute petite surface (puisque les régions alpine et subnivale sont horizontalement très rapprochées de la vallée du Rhòne : $4 \mathrm{~km}$.) que j’ai trouvé à une altitude tout à fait exceptionnelle des espèces qu'on n'avait pas, à ma connaissance, signalées aussi haut.

\title{
CL. GASTROPODA
}

\section{S.-Gl. PULMONATA}

\section{Ord. STYLOMMATOPHORA}

\section{Fam. Vitrinidae.}

\author{
Gen. Limax Müll.
}

\section{Limax (Heynemannia) maximus L.}

Hab. Sous les pierres et le feuillage mort; sur les vieux troncs, sous l'écorce.

D. h. Dans le bas de la vallée: Beuson, Cleibe, Nendaz. Sur les versants : au bord du Bysse de Cleibe et sur les flancs de la montagne de Thyon; dans les forêts de Praz-Condjuz, au-dessus de Haute-Nendaz, sur les flancs de la Dent. Dans le fond de la vallée : Noveli, près de Tortin.

D. v. Jusqu'à $2200^{\mathrm{m}}$ environ à la montagne de Thyon et $2100^{\mathrm{m}}$ au-dessus de Noveli et sur les flancs de la Dent-de-Nendaz.

a) Var. cinereo-niger (Wolff.).

D. v. Partout dans les stations indiquées, de 450 à $2200^{\mathrm{m}}$. 
b) Var. cinerea (List.).

D. h. Comme dans le bas de la vallée; Noveli.

D. v. Jusqu'à environ $1900^{\mathrm{m}}$.

\section{Limax (Heynemannia) tenellus Nils.}

Hab. Dans les forèts, sous les pierres, le feuillage et le bois mort.

D. h. Dans presque toutes les forèts de la vallée : forèts de la montagne de Thyon, forèts de Praz-Condjuz sur les flancs de la Dent-de-Nendaz ainsi qu'aux environs de Bleusy et de Zofleu. Forêts des Eaux.

D. v. Jusqu'à $2200^{\mathrm{m}}$ environ, comme l'espèce précédente.

Gen. Agriolimax Simroth.

3. Agriolimax agrestis (L.).

Hab. Dans les champs ou, comme les autres Limaces, dans les forèts, sous les pierres.

D. h. Très commune dans le bas de la vallée, Aproz, Beuson, Veysonnaz, Cleibe, Nendaz, Haute-Nendaz, Cerisier; plus haut, sur les montagnes: Thyon sur le versant de Nendaz, Zofleu sur les flancs de la Dent-de-Nendaz. Dans le fond de la vallée jusqu'à Tortin.

D. v. Elle atteint, suivant les endroits, 2000 à $2200^{\mathrm{m}}$.

\section{Gen. Vitrina Drap.}

4. Vitrina (Phenacolimax) pellucida (Müll.).

Hab. Sous les pierres, dans les forèts et dans les rocailles de la région subnivale, dans les mèmes conditions que la Vitrina nivalis. 
D. h. Je l'ai trouvée, mais rarement, dans la forêt de PrazCondjuz, sur les flancs de la Dent-de-Nendaz.

D. v. $1300-1400^{\mathrm{m}}$ environ.

\section{Var. alpina Stenz.}

D. h. Sur les bords du glacier du Grand-Désert, au fond du Val-de-Cleuson (leg. Georges de DardeL).

D. v. $2800-2900^{\mathrm{m}}$.

\section{Vitrina (Semilimax) nivalis Charp.}

Hab. Sous de gros blocs de rochers, dans des éboulis parsemés, pendant l'été, de taches de neige.

D. h. A la Dent-de-Nendaz, près du sommet, au-dessus du Lac-Noir. De là, elle suit probablement les bords de la Crête jusqu'au Mont-Gelé; je l'ai trouvée en grande abondance des deux còtés d'un col, entre le Mont-Gond et le Mont-Gelé, sur le versant de Tortin et sur celui du Lac-des-Veaus.

D. v. $2350-2460^{\mathrm{m}}$ à la Dent-de-Nendaz et 2600-2735 (probablement jusqu'à 2800-2900) entre le Mont-Gond et le Mont-Gelé.

\section{Vitrina (Semilimax) diaphana Drap.}

Hab. Dans les forêts, sous les pierres et le bois mort.

D. h. Forêts des flancs de la Dent-de-Nendaz (environs de Bleusy, etc.), de la montagne de Thyon et de Tortin, au fond de la vallée.

D. v. $1300-2200^{\mathrm{m}}$ environ.

7. Vitrina (Semilimax) elongata Drap.

Var. sapinea nov. var.

Ab typo differt apertura multo majore, spira intus multo apertiore, primo anfiactu paulo minore, paulo propiore a latere. Statura major.

Long. 4-6 ${ }^{\mathrm{mm}}$. Lat. 2,75-4 $4^{\mathrm{mm}}$. 
Coquille rhomboïdale, auriculaire allongée, très aplatie, mince, vitreuse, transparente, de couleur verte, mème parfois bleuâtre, surtout à l'intérieur. Spire extrèmement petite, encore plus que chez le type, mais moins que chez la Vitrina brevis, ne faisant nullement saillie, composée de 2 tours s'accroissant très rapidement. Le commencement du premier tour est un peu peu plus rapproché du bord de la coquille que chez le type, mais beaucoup moins que chez la V. brevis. Suture à peine marquée. Ouverture extrèmement grande, très allongée, ovale, à peine échancrée par l'avant-dernier tour. Bord columellaire arqué, brusquement tronqué, avec un rebord relativement large qui en occupe plus de la moitié.

Celte curieuse nouvelle variété est intermédiaire entre les $V$. elongata et brevis, mais bien distincte de cette dernière.

Hab. Comme l'espèce précédente, mais rarement en compagnie immédiate.

D. h. Forèts de Bleusy et des Praz-Condjuz, sur les flancs de la Dent-de-Nendaz; montagne de Thyon, sur le versant de Nendaz.

D. v. $4300-2200^{\mathrm{m}}$, comme l'espèce précédente.

Remarque. Outre les dissemblances constantes de la coquille, l'animal de ces deux dernières Vitrines diffère par le manteau. Celui de la $V$. diaphana est gris unicolore, tandis que le manteau de la $V$. elongata est parsemé de taches noires sur un fond gris.

Gen. Hyalina Fér.

8. Hyalina (Euhyalina) glabra (Stud.).

Hab. Dans les vieux murs ou sous les pierres au bas des haies.

D. h. Dans le bas de la vallée : environs d'Aproz, à Beuson, entre Beuson et Cleibe et jusqu'aux Mayens de Nendaz (à la Biolaz et à Bleusy). 
D. v. Jusqu'à environ $1400^{\mathrm{m}}$ sur le versant de Bleusy et $1300^{\mathrm{m}}$ à Cleibe (cf. ci-après résumé des observations hypsométriques, à propos de la $1^{\text {re }}$ région du Val-Ferret, l'envers et l'endroit).

\section{Hyalina (Euhyalina) cellaria (Müll.).}

Hab. Sous les pierres, au bas des haies, à la lisière des bois.

D. h. Environs d'Aproz.

D. v. Je ne l'ai trouvée qu'à à peu près $500^{\mathrm{m}}$. Il est possible qu'elle vive plus haut, à Beuson ou Cleibe.

\section{Hyalina (Polita) nitens (Mich.).}

Hab. Sous les pierres et le feuillage mort; au bas des haies et dans les forèts.

D. h. Environs d'Aproz, Veysonnaz, Cleibe, Bleusy, Nendaz, Haute-Nendaz et forèts de Praz-Condjuz, sur les flancs de la Dent-de-Nendaz et de la montagne de Thyon.

D. v. Jusqu'à $2200^{\mathrm{m}}$ environ, à la montagne de Thyon ; $2000^{\mathrm{m}}$ environ au-dessus de Nendaz.

\section{Var. dutaillyana (Mabille).}

D. Elle se rencontre avec le ty.pe dans les forèts supérieures de la Dent-de-Nendaz et de Thyon $\left(1500-2200^{\mathrm{m}}\right)$.

\section{Hyalina (Polita) pura (Ald.).}

Hab. Sous les pierres et le bois mort, dans les forèts.

D. h. Dans les forèts de Praz-Condjuz, aux environs de HauteNendaz, environ jusqu'au Bysse de Saxon, au-dessus de Zofleu.

D. v. Jusqu'à environ $1800^{\mathrm{m}}$.

\section{Hyalina (Polita) radiatula Gay.}

Hab. Comme l'espèce précédente et parfois dans des pàturages, sous des grosses pierres (Siviez).

D. h. Dans le bas de la vallée (Veysonnaz), dans les forèts de 
la Dent-de-Nendaz (au-dessus de Zofleu, au bord du Bysse de Saxon et plus haut), et jusqu'à Siviez, près de Tortin.

D. v. Jusqu'à $1950^{\mathrm{m}}$ à Siviez et peut-être un peu moins haut, au-dessus de Zofleu.

\section{Fam. Naninidæ.}

Gen. Euconulus Reinh.

\section{Euconulus fulvus (Müll.).}

Hab. Sous les pierres, le bois mort, le feuillage, etc.; dans les pâturages, sous les pierres, et dans les rocailles de la région alpine.

D. h. Environs d'Aproz; montagne de Thyon, jusqu'à la limite supérieure des forêts; forêts de Praz-Condjuz, Zofleu, bords du Bysse de Saxon, forêts des Eaux, forêts de Bleusy et jusqu'au sommet de la Dent-de-Nendaz; Siviez et Tortin, sur le chemin du Lac-des-Veaus.

D. v. $2100-2200^{\mathrm{m}}$ environ au-dessus de Tortin, $2200^{\mathrm{m}}$ à la montagne de Thyon et $2467^{\mathrm{m}}$ à la Dent-de-Nendaz.

\section{Fam. Arionidæ.}

Gen. Arion Fér.

\section{Arion empiricorum Fér.}

Hab. Surtout dans les champs et les jardins du bas de la vallée; parfois dans les forêts, sous les pierres et le bois mort.

D. h. Dans le bas de la vallée : environs de Aproz, Brignon, Beuson, Nendaz, La Crêta, Cerisier, Haute-Nendaz, entre BasseNendaz et les Mayens de la Biolaz; jusque dans les forêts de Praz-Condjuz (mut. aurantiaca).

D. v. Jusqu'à $1350^{\mathrm{m}}$ environ (Praz-Condjuz).

15. Arion subfuscus (Drap.).

Hab. Dans le bas de la vallée cette espèce vit dans les mêmes conditions que l'Arion empiricorum; plus haut, elle est fréquente 
dans les forêts, sous les grosses pierres moussues, sur les vieux troncs, sous le bois mort, etc. Elle se rencontre enfin, dans les régions alpine et subnivale, sous les grosses pierres, dans les rocailles plus ou moins herbeuses et au pied des parois de rocher.

D. h. Environs d'Aproz, Haute-Nendaz, peu commun dans le bas de la vallée. Sur les flancs de la montagne de Thyon, jusqu'au sommet. Dans les forêts de la Dent-de-Nendaz: les Praz-Condjuz et forêts des environs de Bleusy; jusqu'au sommet de la Dent. Dans le fond de la vallée: Tortin, sur le chemin du Lac-des-Veaus et jusqu'au col entre le Mont-Gond et le Mont-Gelé.

D. v. Il atteint $2467^{\mathrm{m}}$ à la Dent-de-Nendaz et $2735^{\mathrm{m}}$ au-dessus de Tortin ; aux environs de celte dernière localité il est commun jusqu'à à peu près $2350^{\mathrm{m}}$. Il vit encore dans la région subnivale.

\section{Arion hortensis Fér.}

Hab. Dans les régions inférieures et supérieures des vallées et des forêts, cette espèce se rencontre dans les mêmes conditions que l'Arion subfuscus.

D. h. Comme dans le bas de la vallée: environs d'Aproz, Nendaz, Cerisier, Mayens-de-Nendaz, etc. Forêts de la montagne de Thyon, de Praz-Condjuz et des environs de Bleusy.

D. v. Jusqu'à $2200^{\mathrm{m}}$ à la montagne de Thyon.

\section{Fam. Polyplacognatha.}

Gen. Punctum Morse.

\section{Punctum pygmaeum (Drap.).}

Hab. Sur les gros blocs moussus et plus rarement sous les pierres et le feuillage mort; dans les rocailles de la région alpine. 
D. h. Environs d'Aproz, montagne de Thyon sur les bords du Bysse de Verrey, du Bysse de Thyon; sur les bords du Lac Noir, au pied de la Dent et jusqu'au sommet de la Dent-deNendaz.

D. v. $1800^{\mathrm{m}}$ au Bysse de Thyon, $2200^{\mathrm{m}}$ au Lac Noir et $2467^{\mathrm{m}}$ à la Dent.

\section{Fam. Patulidae.}

Gen. Pyramidula Fitz.

\section{Pyramidula rupestris (Drap.).}

Hab. Dans les régions inférieures, contre les rochers et dans les régions alpine et subnivale, sous les rocailles, les gros blocs de pierre et contre les petites parois de rocher bien abritées.

D. h. Environs d'Aproz et sur le chemin de Basse-Nendaz; probablement dans d'autres stations des régions inférieures. Dent-de-Nendaz, sur le versant du Lac Noir, jusqu'au sommet. Tortin, sur le chemin du Lac-des-Veaus et jusqu'au col entre le Mont-Gelé et le Mont-Gond. Près du glacier du GrandDésert, au fond du Val-de-Cleuson (de Dardel leg.).

D. v. $2300-2467^{\mathrm{m}}$ à la Dent-de-Nendaz; $2300-2735^{\mathrm{m}}$ entre les monts Gond et Gelé et 2800-2900 ${ }^{\mathrm{m}}$ au Grand-Désert.

\section{Gen. Patula Held.}

\section{Patula (Discus) rotundata (Müll.).}

Hab. Dans les forèts inférieures, sous les pierres et le feuillage mort, sur les vieux trones; à la lisière des bois et au pied des haies, sous les pierres.

D. h. Dans le bas de la vallée seulement: environs d'Aproz, Veysonnaz, Cleibe, Beuson, Nendaz, jusqu'au Mayen de la Biolaz et après Bleusy.

D. v. Elle n'atteint que $1250^{\mathrm{m}}$ à Veysonnaz, $1350^{\mathrm{m}}$ à la Biolaz et à peu près $1400^{\mathrm{m}}$ près de Bleusy. 


\section{Patula (Discus) ruderata (Stud.).}

Hab. Dans les forèts, sur les vieux troncs, les blocs moussus (en particulier la var. viridana), sous les pierres et le bois mort.

D. h. Forèts des environs de Haute-Nendaz(Praz-Condjuz, etc.), au-dessus de Zofleu, sur les bords du Bysse de Saxon, dans les forèts de Bleusy et des Eaux; montagne de Thyon.

D. v. De $1200^{\mathrm{m}}$ (Haute-Nendaz) à $2200^{\mathrm{m}}$ ( $\mathbf{M}^{\mathrm{t}}$-de-Thyon).

\section{Var. viridana Roff.}

D. h. Dans les forêts du pied de la Dent-de-Nendaz, aux environs de Bleusy.

D. v. $1350-1500^{\mathrm{m}}$ environ.

\section{Fain. Eulotidae.}

Gen. Eulota (Htm.).

\section{Eulota fruticum (Müll.).}

Hab. Dans les champs, au pied des arbustes et des haies.

D. h. Cerisier, la Crète, Haute-Nendaz et dans le vallon jusqu'à la forèt de Praz-Condjuz.

D. v. Jusqu'à $1350^{\mathrm{m}}$ environ.

\section{Fam. Helicidae.}

S.-Fam. Vallonitiea.

Gen. Vallonia Risso.

22. Vallonia pulchella (Müll.).

Var. helvetica (Sterki).

Hab. Dans les champs au bord des chemins, sous les pierres et le bois mort. 
D. h. Environs d'Aproz, de Beuson et de Cleibe.

D. v. Jusqu'à environ $1250^{\mathrm{m}}$.

S.-Fam. Helicodontinae.

Gen. Helicodonta Risso.

23. Helicodonta (Trigonostoma) obvoluta (Müll.).

Hab. Dans les mêmes conditions que la Patula rotundata.

D. h. Environs d'Aproz, Veisonnaz, Cleibe, Beuson, forêts de Praz-Condjuz. Dans cette forêt, les Helicodonta holoserica et obvoluta vivent en compagnie immédiate, parfois même sous la même pierre. J'ai trouvé l'H. holoserica le long d'un chemin au bas d'une forte pente au sommet de laquelle vit l' $/$. obvoluta.

D. v. Jusqu'à environ $1350^{\mathrm{m}}$.

\section{Helicodonta (Trigonostoma) holoserica (Stud.).}

Hab. Dans les forèts supérieures, sous les grosses pierres et parfois sous le bois mort.

D. h. Montagne de Thyon; forêts de Praz-Condjuz, de Bleusy et des Eaux, sur les flancs de la Dent-de-Nendaz.

D. v. Jusqu'à $2200^{\mathrm{m}}$ environ.

\section{S.-Fam. Fruticicolinae.}

Gen. Fruticicola Held.

25. Fruticicola (Fruticicola s. str.) sericea (Drap.).

Hab. Dans les forêls, sur les vieux troncs; à la lisière des forêts et au bord des chemins, au pied des haies, sous lespierres et le bois mort.

D. h. Environs d'Aproz, Brignon, Beuson, Cleibe, Veysonnaz, 
Basse-Nendaz, Haute-Nendaz et Mayen de la Biolaz; forèts de Praz-Condjuz.

D. v. Jusqu'à $1350^{\mathrm{m}}$ environ.

26. Fruticicola (Euomphalia) strigella (Drap.).

Hab Au bord des chemins, dans les petits bois, au pied des haies; sous le feuillage mort et les pierres.

D. h. Environs d'Aproz et dans le bas de la vallée jusqu'aux Mayens de Nendaz.

D. v. Jusqu'à $1350^{\mathrm{m}}$, comme la F. sericea.

\section{S.-Fam. Campylaeinae.}

Gen. Arianta Leach.

\section{Arianta arbustorum (L.)}

Hab. Dans les champs, aux endroits humides, et dans les forêts.

D. h. Environs d'Aproz, Beuson, Cleibe, Nendaz, HauteNendaz.

D. v. Jusqu'à environ $1300^{\mathrm{m}}$.

\section{Var. alpicola Charp.}

Hab. Dans les forèts, les pàturages supérieurs et les rocailles des régions alpines.

D. h. Forèts de la montagne de Thyon, jusqu'à la Crête; forèt des Eaux et Dent-de-Nendaz, jusqu'au sommet.

D. v. Jusqu'à $2467^{\mathrm{m}}$.

Gen. Chilotrema Leach.

28. Chilotrema lapicida (L.).

Hab. Dans les forêts, sous les pierres, le feuillage mort, sous les vieux troncs, ou dans les endroits humides, au pied des haies, sous les pierres, dans les vieux murs, sous le lierre. 
D. h. Environs d'Aproz, Brignon, Beuson, Veysonnaz, Cleibe jusqu'au bord du "bysse », à la lisière des forêts, Nendaz jusqu’aux Mayens (La Biolaz, etc.); forêts des Praz-Condjuz.

D. v. Jusqu'à $1350^{\mathrm{m}}$ environ.

\author{
S.-Fam. Helicinae. \\ Gen. Helix L. s. str.
}

\title{
29. Helix pomatia L.
}

Hab. Dans les champs, ordinairement en compagnie d'Arianta arbustorum.

D. h. Environs d'Aproz, Beuson, Veysonnaz, Cleibe, Basse et Haute-Nendaz jusqu'aux mayens; en suivant la vallée elle n'arrive guère que jusqu'à la forêt des Eaux.

D. v. Jusqu'à environ $1400^{\mathrm{m}}$.

Remarque. On trouve différentes variations de forme et de couleur (en particulier globosa, elevata, etc.). Au Mayen de la Biolaz, au-dessus de Basse-Nendaz vit une jolie mutation a trois fascies ininterrompues.

Gen. Tachea Leach.

30. Tachea nemoralis (L.).

Hab. Dans les champs et les bois, aux endroits humides.

D. h. Elle ne vit pas précisément dans le Val de Nendaz, mais à son extrémité dans la vallée du Rhòne, aux environs d'Aproz.

D. v. Je ne l'ai pas rencontrée au-dessus de $500^{\mathrm{m}}$.

\section{Tachea sylvatica (Irap.).}

Var. montana (Stud.).

Hab. Sous les pierres, dans les rocailles de la région alpine. 
D. h. Au-dessus du Lac-Noir jusqu'au sommet de la Dent-deNendaz.

D. v. $2300-2467^{\mathrm{m}}$.

\section{S.-Fam. Xerophilinae.}

Gen. Xerophila Held.

32. Xerophila (Xerophila s. str.) obvia (Htm.)

Hab. Dans les champs, au bord des grandes routes.

D. h. Cette espèce n'est pas encore répandue dans le Val-deNendaz, mais il est plus que probable qu'elle suivra tout le parcours de la route qu'on est en train de construire entre Sion et Nendaz, par Salins et Brignon. La X. obvia est actuellement très commune à Sion et aux environs, sous les Agettes à Salins et presque jusqu'au-dessus de Baar, c'est-à-dire dans le Val-de-Nendaz.

D. v. $490-750^{\mathrm{m}}$ environ.

33. Xerophila (Xerophila) ericetorum (Müll.).

Hab. Dans les mêmes conditions que la précédente.

D. h. Je ne l'ai pas trouvée dans le Val-de-Nendaz; elle n'atteint sans doute que sa partie inférieure, jusqu'à Baar, dans la vallée du Rhòne.

D. v. Environ jusqu'à $700^{\mathrm{m}}$.

\section{Xerophila (Striatella) candidula (Stud.).}

Hab. Dans les champs, au bord des chemins, sur les herbes.

D. h. Dans le bas de la vallée jusqu'à Beuson et Cleibe.

D. v. Jusqu'à $1300^{\mathrm{m}}$ environ. 


\section{Fam. Buliminidae.}

Gen. Buliminus Ehr.

\section{Buliminus (Zebrina) detritus (Müll.).}

Hab. Dans les mèmes conditions que la X. candidula.

D. h. Aussi dans le bas de la vallée, jusqu'à Cleibe et Beuson.

D. v. Jusqu'à $1300^{\mathrm{m}}$ environ.

36. Buliminus (Ena) montanus Drap.

Hab. Dans les forèts, sur les vieux troncs et les blocs moussus ainsi que sous les pierres et le bois mort.

D. h. Dans les forèts de Praz-Condjuz.

D. v. Jusqu'à environ $1500^{\mathrm{m}}$.

\section{Buliminus (Ena) obscurus (Müll.).}

Cette espèce se trouve dans les forêts de Praz-Condjuz, dans les mèmes conditions biologiques et hypsométriques que la précédente.

Gen. Chondrula Beck.

38. Chondrula quadridens (Müll.).

Hab. Dans les champs, sous les pierres et le bois mort.

D. h. Dans le bas de la vallée: Beuson, Cleibe, Veysonnaz, Sornard, Mayens de Nendaz (la Biolaz, etc.), et au-dessus de Haute-Nendaz.

D. v. Jusqu'à environ $1350^{\mathrm{m}}$. 
Fam. Cochlicopidae.

Gen. Cochlicopa Risso.

39. Cochlicopa lubrica (Müll.).

Hab. Elle vit souvent dans les mèmes conditions que l'espèce précédente; on la trouve, en outre, dans les forèts, sous les pierres et le bois mort, sur les vieux troncs et les blocs moussus.

D. h. Environs d'Aproz, de Beuson, Cleibe et dans les forèts de Praz-Condjuz.

D. v. Jusqu'à environ $1500^{\mathrm{m}}$.

\section{Fam. Pupidae.}

Gen. Pupa Drap.

40. Pupa (Modicella) avenacea (Brug.).

Hab. Contre les rochers et sur les vieux murs.

D. h. Je ne l'ai trouvée qu'à Aproz.

D. v. Environ $490-500^{\mathrm{m}}$ :

Gen. Pupilla Leach.

\section{Pupilla muscorum (L.).}

Hab. Dans les champs, sous les pierres et le bois mort.

D. h. Environs d'Aproz, de Beuson, jusqu'à Cleibe.

D. v. Jusqu'à environ $1300^{\mathrm{m}}$.

\section{Pupilla triplicata (Stud.).}

D. Je l'ai trouvée à Beuson et à Cleibe, dans les mèmes conditions biologiques et hypsométriques que l'espèce précédente. 


\section{Pupilla alpicola (Charp.).}

Var. saxetana nov. var.

Ab typo differt tesla brevissima, lala, valde ventricosa, minute striata; sutura normalis, eadem dentulina. Anfractus 5, valde convexi.

Long. 2,25-2,75 mm . Lat. 1,50-1,75 ${ }^{\mathrm{mm}}$.

Hab. Contre les rochers et sous les pierres.

D. h. J'ai recueilli une vingtaine d'exemplaires de cette nouvelle variété sur les bords du lac des Veaus et quelques individus au sommet du col entre le Mont-Gond et le Mont-Gelé.

D. v. $2550-2735^{\mathrm{m}}$.

Test présentant une fente ombilicale assez longue et dilatée sur les bords, finement et régulièrement strié, un peu plus que chez le type, très court et large, très ventru, ovale cylindrique, très peu brillant, de couleur brune; sommet très arrondi. Seulement 5 tours de spire, très convexes, s'accroissant plus rapidement que le type; suture profonde. Ouverture assez grande, de forme normale, arrondie. Péristome légèrement bordé de blanc à l'intérieur, assez mince; bord columellaire peu oblique, étroit, mieux bordé que les autres. Pas de bourrelet extérieur. Une très petite dent pariétale à peine perceptible, faisant souvent défaut.

\section{Gen. Vertigo (Müll.).}

\section{Vertigo (Alaea) pygmaea (Drap.).}

Hab. Sur des blocs granitiques moussus.

D. h. Je ne l'ai trouvée qu'à Beuson.

D. v. Environ $960-970^{\mathrm{m}}$.

\section{Vertigo (Alaea) alpestris (Ald.).}

Hab. Sur les blocs moussus; sous les pierres, dans les rocailles de la région alpine. 
D. h. Forêts au-dessus de Veysonnaz, forêts de Praz-Condjuz et des Eaux; Dent-de-Nendaz, jusqu'au sommet.

D. v. $1300-2467^{\mathrm{m}}$.

\section{- Fam. Glausiliidae.}

Gen. Balea Prid.

46. Balea perversa (L.).

Hab. Sur les vieux troncs et les blocs moussus.

D. h. Veysonnaz, sur les bords du Bysse de Verrey (Bysse de Thyon), au-dessus de Cleibe, sur les flancs de la montagne de Thyon, Beuson, Mayen de la Biolaz (sur Basse-Nendaz), forêts de Bleusy et de Praz-Condjuz.

D. v. $750-1800^{\mathrm{m}}$.

Gen. Clausilia Drap.

\section{Clausilia (Cusmicia) dubia Drap.}

Hab. Sur les vieux troncs, les blocs moussus, sous les pierres.

D. h. Montagne de Thyon, dans toute la forêt; forêts après Bleusy, sur les flancs de la Dent-de-Nendaz; forêts de PrazCondjuz.

D. v. Cette espèce atteint $2200^{\mathrm{m}}$ à la montagne de Thyon.

\section{Var. alpicola Clessin.}

Hab. Dans les rocailles de la région alpine.

D. h. Dans la partie supérieure de la Dent de Nendaz et jusqu'au sommet.

D. v. De 2300 à $2467^{\mathrm{m}}$. Les exemplaires valaisans de cette variété sont fort typiques et semblables a ceux de Schlern (Tyrol: $2560^{\mathrm{m}}$ ). 
48. Clausilia (Pirostoma) plicatula Drap.

Hab. Sur les vieux troncs et les blocs moussus; sous les pierres et dans les vieux murs.

D. h. Aproz, Veysonnaz, Beuson, Cleibe et en général dans tout le bas de la vallée; forèts de Praz-Condjuz et à Zofleu, jusqu'au Bysse de Saxon.

D. v. Jusquà environ $1800^{\mathrm{m}}$.

\section{Clausilia (Pirostoma) ventricosa Drap.}

Hab. Au pied des haies, sous le bois et le feuillage morts, dans les lieux très humides.

D. h. Un peu aı-dessus de Beuson du côté de Sornard; entre Cleibe et Veysonnaz.

D. v. Jusqu'à $1300^{\mathrm{m}}$ environ.

\section{Fam. Succineidae.}

Gen. Succinea Drap.

50. Succinea (Amphibina) pfeifferi Rossm.

Hab. Au bord des mares et des ruisseaux.

D. h. Environs d'Aproz et de Haute-Nendaz.

D. v. $490-1300^{\mathrm{m}}$ environ.

\section{Succinea (Lucena) oblonga Drap.}

Hab. Dans les prairies très humides.

D. h. Entre Beuson et Cleibe.

D. v. Jusqu'à $1300^{\mathrm{m}}$ environ. 


\section{Ord. BASOMMATOPHORA.}

\section{Fam. Limnaeidae.}

Gen. Limnaea (Lam.).

52. Limnaea (Gulnaria) limosa (L.), sensu latissimo.

$$
\text { Subsp. peregra (Müll.). }
$$

Hab. Dans les mares garnies de plantes aquatiques et profondes de $0^{\mathrm{m}}, 50-1^{\mathrm{m}}$.

D. h. Au-dessus de Hautè-Nendaz.

D. v. A environ $1350^{\mathrm{m}}$.

\section{Var. excerpta Hartm.}

Hab. Dans des flaques boueuses profondes de $0^{\mathrm{m}}, 10-0^{\mathrm{m}}, 20$, dans une eau en général très sale.

D. h. A Ouché, dans le vallon de Cleuson.

D. v. $2000^{\mathrm{m}}$ environ.

53. Limnaea (Fossaria) truncatula (Müll.).

Hab. Dans des mares, des ruisseaux et des bassins de fontaines.

D. h. Environs de Veysonnaz et de Haute-Nendaz; Siviez et aux Sept-Fontaines (près du Lac-Noir, au pied de la Dent-deNendaz).

D. v. $1950^{\mathrm{m}}$ à Siviez et $2000^{\mathrm{m}}$ environ près des Sept-Fontaines. 


\section{ACEPHALA}

\section{Fam. Sphaeriidae.}

Gen. Pisidium C. Pfr.

\section{Pisidium (Fossarina) fossarinum $\mathrm{Cl}$.}

Hab. Dans des mares, des flaques, des ruisseaux et des petits lacs alpestres, enfouie à quelques centimètres de profondeur, dans la vase.

D. h. Mares au-dessus de Haute-Nendaz, ruisseau à Zofleu, Lac-Noir (au pied de la Dent-de-Nendaz); Siviez et Praz-Fleuri (près de Tortin); près de Cleuson. Dans des petits lacs à la Crète-de-Thyon.

D. v. Jusqu'à $2200^{\mathrm{m}}$ au Lac-Noir, $1950-2000^{\mathrm{m}}$ aux environs de Tortin, $2100^{\mathrm{m}}$ environ près de Cleuson et $2200^{\mathrm{m}}$ à la Crète-deThyon.

\section{APPENDICE}

J'ai recueilli, en dehors des limites du Val de Nendaz et de la vallée suivante, un certain nombre d'espèces qu'il me paraît intéressant de mentionner.

I. A Sion, sur la colline de Tourbillon, sur le versant sud, j'ai retrouvé le Pupa granum Drap., signalé par Charpentier en 1837; il y est assez rare. En outre, j'ai recueilli 5 ou 6 exemplaires du Pupa variabilis Drap., déjà observé par le major Le Hon ${ }^{1}$. La Xerophila candidula var. gratiosa (Stud.) est très répandue sur ce versant, où elle atteint une taille remarquable (diam. $11^{\mathrm{mm}}$, alt. $7^{1 / 2}$ ). Les Buliminus detritus (Müll.), Chondrula quadridens (Müll.), Pupa avenacea (Brug), Pupilla

1 Voir Roffinen, Mollusques terrestres et fluviatiles recueillis en suisse. Ann. de la Soc. malac. de Belgique, III, 1868. 
muscorum (L.), Isthmia muscorum (Drap.), Tachea sylvatica (Drap.), Chilotrema lapicida (L.), Fruticicola strigella (Dr.), Vallonia costata (Müll.), Pyramidula rupestris(Dr.) sont également très communs.

II. Non loin de Sion, sur le chemin d'Aproz, on trouve dans des mares et des fossés, plusieurs espèces aquatiques intéressantes :

1. Limnaea (Limnus) stagnalis (L.).

2. Limnaea (Gulnaria) limosa (L.) sensu latiore, subsp. peregra (Müll)., var. compressa Hartm. (fig. 14́.).

3. Limnaea(Limnophysa)palustris (Müll.) etvar.corvus (Gm.).

4. Limnaea (Fossaria) truncatala (Müll.).

5. Aplexa hypnorum (L.).

6. Planorbis (Tropidiscus) marginalus (Dr.).

7. Planorbis (Bathyomphalus) contorlus (L.).

8. Planorbis (Armiger) crista (L.) var. cristala (Drap.).

9. Planorbis (Hippeutis) complanatus (L.).

10. Valvata (Gyrorbis) cristata Müll.

11. Pisidium (Fossarina) obtusale C. Pfr.

Sur les bords des mares et des ruisseaux :

12. Succinea (Amphibina) pfeifferi Rossm.

III. Près du Col des Etablons, entre la vallée de Bagne (Châble) et le Val d'Isérable, j’ai trouvé à environ $2150^{\mathrm{m}}$ d'altitude le Pisidium fossarinum Cl. et la Limnaea limosa (L.) subsp. peregra (Müll.), dans des pàturages très marécageux. L'Arianta arbustorum var. alpicola s'y trouve jusqu'à $2200^{\mathrm{m}}$.

\section{IV}

Mollusques récoltés au Val dHérens.

Ce chapitre est le résultat des observations que j’ai pu faire sur 55 espèces du Val d'Hérens; j’ai déjà donné au «Rameau 
de Sapin " (1911, pp. 30, 40 et 46) une liste de 25 espèces recueillies dans la partie supérieure de cette vallée. Je dois en outre des remerciements à mon ami M. Reichel, qui m'a rapporté des coquilles intéressantes de son séjour aux Haudères.

\section{GASTROPODA}

\section{S.-Gl. PULMONATA}

\section{Ord. STYLOMMATOPHORA}

\section{Fam. Vitrinidæ.}

\section{Gen. Limax Müll.}

\section{Limax (Heynemannia) maximus L.}

Hab. Dans les forèts, sous les pierres et le bois mort.

D. h. Environs de Bramois, de l'Ermitage de Longeborgne, Nax, Mase (ou Mage), Vex, Hérémence, les Mayens de Sion (extrémité orientale, au-dessus de Vex), forêts de Thion, environs d'Evolène, des Haudères, dans les forêts de Veisivi, d'Arolla et de la Combe de Ferpècle.

D. v. Jusqu'à $2100^{\mathrm{m}}$ à Arolla, à près de $1800^{\mathrm{m}}$ à Ferpècle.

a) Var. cinereo-niger (Wolff.).

D. h. Répandue partout.

D. v. Jusqu'à $2100^{\mathrm{m}}$.

b) Var. cinerea (List.).

D. h. Répandue surtout dans le bas de la vallée, Vex, etc.

D. v. Jusqu'à $1400^{m}$ au plus.

\section{Limax (Heynemannia) tenellus Nils.}

Hab. Dans les forêts, sous les pierres ou sur les vieux troncs; dans les alpages supérieurs, sous les rocailles. 
D. h. Dans les régions supérieures de la vallée : forêts de la montagne de Thyon au-dessus d'Hérémence, environs d'Evolène, des Haudères et dans les Combes d'Arolla et de Ferpècle.

D. v. $1300-2100^{\mathrm{m}}$ environ.

Gen. Agriolimax Simr.

3. Agriolimax agrestis (L.).

Hab. Dans les champs et les forèts.

D. h. Elle pullule dans toute la vallée, de Bramois à Arolla et Ferpècle.

D. v. $490-2100^{\mathrm{m}}$.

Gen. Vitrina Drap.

4. Vitrina (Semilimax) elongata Drap.

Var. sapinea Piaget.

Hab. Sous les pierres et le feuillage mort; sous le bois pourri.

D. h. Forêts de Veisivi, au-dessus des Haudères. Elle vit sur la montagne de Thyon, mais je ne l'y ai pas rencontrée sur le versant du Val d'Hérens.

D. v. $1450-1700^{\mathrm{m}}$ environ.

Remarque: Dans l'article mentionné plus haut (p. 31), j'ai parlé de cette espèce sous le nom de $V$. diaphana. d’après la détermination de M. Paul Gover.

\section{Vitrina (Semilimax) diaphana Drap.}

Hab. Dans les forêts, sous les pierres et le bois mort.

D. h. Je ne l'ai trouvée qu'aux environs d'Hérémence, Mayens de Sion et montagne de Thyon, mais elle doit être plus répandue.

D. v. Jusqu'à environ $1950^{\mathrm{m}}$. 
6. Vitrina (Phenacolima.x) pellucida (Müll.).

Hab. Sous les pierres, le bois et le feuillage mort; dans les forêts ou les lieux très humides.

D. h. Dans le bas de la vallée: Bramois, Ermitage de Longeborgne et Nax; jusqu'à Luette.

D. v. Jusqu'à $1300^{\mathrm{m}}$.

\section{Vitrina (Phenacolimax) annularis Venetz.}

Hab. Sous les cailloux et le feuillage mort.

D. h. Un peu au-dessous de Nax, dans une forèt.

D. v. Environ $1200^{\mathrm{m}}$.

Remarque: Cette espèce n'est pas, comme le dit Cuessin, caractéristique des régions alpine ou subnivale. Je l'ai trouvée dans la région inférieure des forêts et suis parfaitement sûr de ma détermination. Mon unique exemplaire est tout à fait typique, il a de fortes stries disposées en anneaux, une spire plus élevée que la $V$. pellucida, un dernier tour plus étroit et une spire s'accroissant moins rapidement. Du reste, Locaro la signale en France dans des régions qui sont loin d'ètre alpines.

Gen. Hyalina Fér.

8. Hyalina (Euhyalina) glabra (Stud.).

Hab. Sous les pierres et le bois mort; sur des rochers humides ou entre les pierres des murs de clòture.

D. h. Dans le bas de la vallée: Bramois, Vex, Ermitage de Longeborgne, Nax, Vernamiège, Mage et la Luette.

D. v. Jusqu'à $1000^{\mathrm{m}}$ dans le fond de la vallée, jusqu'a $1400^{\mathrm{m}}$ sur les flancs $\left(1200^{\mathrm{m}}\right.$ à Vex $)$.

\section{Hyalina (Polita) nitens (Mich.).}

Hab. Sous les pierres et le bois mort.

D. h. Commune dans toute la vallée: Bramois, Nax, Vex, 
Mayens de Sion, Mase, Suin, St-Martin, Luette, environs d'Evolène et des Haudères, Getty, Arolla et Ferpècle.

D. v. Jusqu'à environ $1950^{\mathrm{m}}$.

\section{Var. dutaillyana (Mab.).}

D. h. Dans la partie supérieure de la vallée: environs des Haudères et montagne de Thyon.

D. v. $1400-1950^{\mathrm{m}}$.

\section{Hyalina (Polita) pura Ald.).}

Hab. Sous le feuillage mort, sur les vieux troncs et les blocs moussus.

D. h. Dans les forèts du bas de la vallée: Mayens de Sion, etc.

D. v. Jusqu'à 1300-1350 ${ }^{\mathrm{m}}$ environ.

\section{Hyalina (Polita) radiatula Gray.}

Hab. Sous les pierres, le feuillage mort, et sur des rochers moussus et humides.

D. h. Dans le bas de la vallée, comme l'espèce précédente: Mayens de Sion, la Luette, etc.

D. v. Jusqu'à $1000^{\mathrm{m}}$ dans le fond de la vallée et jusqu'à $1350^{\mathrm{m}}$ sur les flancs.

\section{Fam. Naninidae.}

Gen. Euconulus Reich.

\section{Euconulus fulvus (Müll.)}

Hab. Sous les pierres, le bois et le feuillage morts.

D. h. Dans la plupart des forêts: Mayens de Sion, Nax, Luette, Evolène, Getty, Haudères, Arolla et Ferpècle.

D. v. Dans les forèts elle atteint $1950-2000^{\mathrm{m}}$, au fond de la combe de Ferpècle elle atteint $1800^{\mathrm{m}}$ et $2100^{\mathrm{m}}$ dans le vallon d'Arolla. 


\section{Fam. Arionidae.}

Gen. Arion Fér.

\section{Arion empiricorum Fér.}

Hab. Dans les forêts, sous les pierres ou sur les vieux troncs; dans les champs.

D. h. Dans tout le fond de la vallée, jusqu'aux Haudères (mut. atra à Vex).

D. v. Jusqu'à $1450^{\mathrm{m}}$ environ.

\section{Arion subfuscus (Drap.).}

Hab. Dans les champs et les forèts, sous les pierres, le bois mort, etc.; dans les alpages supérieurs.

D. h. Disséminée dans toute la vallée: Bramois, Mayens de Sion au-dessus de Vex, Suin, Hérémence, forèts des Haudères, Ferpècle et Arolla.

D. v. Je ne l'ai trouvée que jusqu'à $2300^{\mathrm{m}}$.

\section{Arion hortensis Fér.}

Hab. Dans les champs et les forêts, sous les pierres, le bois et le feuillage morts, sous la mousse, sur les vieux troncs et les blocs moussus, etc.

D. h. Dans toute la vallée, disséminée un peu partout.

D. v. Jusqu'à environ $1950^{\mathrm{m}}$.

Fam. Polyplacognatha.

Gen. Punctum Morse.

16. Punctum pygmaeum (Drap.).

Hab. Sous le feuillage mort et sur les gros blocs moussus.

D. h. Forêts entre les Mayens de Sion et Hérémence.

D. v. Jusqu'à $1500^{\mathrm{m}}$ environ. 


\section{Fam. Patulidae.}

Gen. Pyramidula Fitz.

\section{Pyramidula rupestris (Drap.).}

Hab. Contre les rochers ou sous des pierres plates.

D. h. Très disséminée: Bramois, Vex, forèt près de Gétty.

D. v. Jusqu'à $1900^{\mathrm{m}}$.

\section{Gen. Patula Held.}

18. Patula (Discus) rotundata (Müll.).

Hab. Sous les pierres et le feuillage mort; contre les rochers.

D. h. Dans le bas de la vallée : Bramois, Ermitage de Longeborgne, Mayens de Sion. Nax, Vernamiège, la Luette.

D. v. Seulement jusqu'à $1400^{\mathrm{m}}$.

\section{Patula (Discus) ruderata (Stud.).}

Hab. Sous le bois mort et les grosses pierres, dans les forêts seulement.

D. h. Dans toutes les forèts de la région supérieure: Mayens de Sion et montagne de Thyon, au-dessus d'Evolène, les Haudères, Ferpècle et Arolla.

D. v. Jusqu'à environ $2000-2100^{\mathrm{m}}$, depuis $1300^{\mathrm{m}}$.

\section{Var. viridana Roff.}

Hab. Sur de gros blocs granitiques, moussus et garnis de lichens.

D. h. Au-dessus des Haudères.

D. v. $1500-1700^{\mathrm{m}}$. 


\section{Fam. Helicidae.}

S.-Fam. Valloninae.

Gen. Vallonia Risso.

20. Vallonia pulchella (Müll.).

Var. helvetica (Sterki).

Hab. Sous les pierres, au bord des chemins, dans les champs.

D. h. Entre Bramois et Nax, Euseigne et la Luette.

D. v. Jusqu'à $1300^{\mathrm{m}}$ sur le versant de Nax, jusqu'à $1050^{\mathrm{m}}$ dans le fond de la vallée.

S.-Fam. Helicodontinae.

Gen. Helicodonta Risso.

\section{Helicodonta (Trigonostoma) obvoluta (Müll.).}

Hab. Sous les pierres et le bois mort.

D. h. Dans le bas de la vallée : environs de Bramois et de Vex.

D. v. A peine jusqu'à $1000^{\mathrm{m}}$. Cette limite entre les Helicodonta obsoluta et holoserica est très curieuse et particulière au Val d'Hérens. Ailleurs, on trouve les deux espèces réunies à $1300^{\mathrm{m}}$ et, sur un espace d'environ 50 mètres en hauteur, se trouvent la limite supérieure de la première espèce et la limite inférieure de la seconde. Au Val d'Hérens, à exactement $1000^{\mathrm{m}}$, au bord de la Borgne, à 20 mètres au-dessous du hameau de la Luette $\left(1020^{\mathrm{m}}\right)$ je n'ai trouvé que des Helicodonta holoserica, sans aucune trace de sa correspondante des régions inférieures, et en compagnie d'Arianta arbustorum normales et (non var. alpicola), de Vallonia helvetica, de Hyalina radialula, Vitrina pellucida, Pupilla Iriplicata et Clausilia ventricosa, c'est-à-dire autant d'espèces qu'on n'a pas l'habitude de trouver réunies. 


\section{Helicodonta (Trigonostoma) holoserica (Stud.).}

Hab. Dans les forèts alpines, sous les gros cailloux humides, souvent assez profondément dans des trous.

D. h. Dans la région supérieure de la vallée, depuis la Luette, Mayens de Sion, montagne de Thyon, au-dessus d'Evolène, forèts des Haudères, de Ferpècle et d'Arolla.

D. v. $1000(!)-1900^{m}$ environ ; contrairement à ce qui arrive dans les quatre autres vallées que j'ai explorées, cette espèce descend dans le fond du Val d'Hérens $300^{\mathrm{m}}$ plus bas que la Patula ruderata. Mais il est à remarquer que sur le versant des montagnes, aux Mayens de Sion par exemple, on ne la rencontre jamais au-dessous de $1300^{\mathrm{m}}$.

\section{S.-Fam. Fruticicolinae.}

\section{Gen. Fruticicola Held.}

\section{Fruticicola (Fruticicola s. str). sericea (Drap.).}

Hab. Sous le bois et le feuillage morts; sous les pierres.

D. h. Disséminée dans toute la vallée : environs de Bramois, Ermitage de Longeborgne, Nax, Vex, Mage, Suin, la Luette, etc. jusqu'à la forêt de Veisivi, au-dessus des Haudères.

D. v. Jusqu'à environ $1900^{\mathrm{m}}$.

\section{Fruticicola (Fruticicola) hispida (L.).}

Hab. Sous les pierres et le bois mort.

D. h. Bas de la vallée. environs de Bramois et de Nax.

D. v. Jusqu'à $1300^{m}$ environ.

Remarque : La distribution de cette espèce, en Suisse, donne lieu, depuis longtemps, à de longues discussions. Charpentier, en 1837, ne la signale que sur le Plateau. En 1868, Rofriarn la 
découvre dans un certain nombre de localités des Alpes Martigny, Wesen, Viège, Grande Scheideck, etc.). En 1887, Clessin la donne comme se trouvant dans toute la Suisse et toute l'Autriche-Hongrie sauf le Tyrol et la Transsylvanie. En 1907, Godet dit qu'elle n'a pas été trouvée dans le canton de Neuchâtel et qu'elle paraît préférer les terrains molassiques. Mais en 1909, Bollinger, relève une quantité de provenances jurassiennes, y compris Biaufond et Valanvron, et donne à cette espèce une distribution en Suisse bien plus étendue que ne la concevait Godet; j'ai chez moi des notes que ce dernier auteur a réunies sur les Mollusques suisses, et dans une copie du travail de Roffiaes il a noté en marge qu'il devait y avoir erreur de détermination et que la $F$. hispida de l'auteur belge devait ètre une variété de sericea ou de plebeja. Quant à ma Fruticicola du bas du Val d'Hérens, je ne vois guère comment il l'aurait déterminée, avec son très large et profond ombilic et sa toute petite taille qui empèche absolument de la rapporter à une $F$. coelata ou montana. D'autre part, elle est absolument semblable aux $F$. hispida des bords du lac d'Annecy, du midi de la France, du Plateau suisse et de la Franche-Comté.

\section{Fruticicola (Euomphalia) strigella (Drap.).}

Hab. Sous le bois et le feuillage morts, à la lisière des forèts. Mais à la Forclaz je l'ai trouvée sous les pierres, dans une forêt, en pleine région supérieure, ce qui avait beaucoup étonné M. Paul Godet.

D. h. Environ de Bramois, Nax, Vernamiège, environs de Vex, et jusqu'à la Forclaz, au-dessus des Haudères.

D. v. Jusqu'à $1700^{\mathrm{m}}$ environ à la Forclaz; jusqu'à $1350^{\mathrm{m}}$ sur les flancs des montagnes, au bas de la vallee (Nax et Vex). 


\section{S. -Fam. Campylaeinae.}

Gen. Arianta Leach.

\section{Arianta arbustorum (L.).}

Hab. Dans les champs et les forêts, sous le feuillage mort, les pierres, le bois pourri, etc.

D. h. Seulement dans le bas de la vallée: Bramois, Nax, Vernamiège, Vex, Mayens de Sion, Hérémence; dans le fond de la vallée jusqu'à Luette où elle vit avec l'Helicodonta holoserica.

D. v. Jusqu'à $1300^{\mathrm{m}}$ sur les flancs des montagnes: Nax, Vernamiège et Mayens de Sion, mais seulement jusqu'à $1000^{\mathrm{m}}$ dans le fond de la vallée.

\section{Var. alpicola Charp.}

Hab. Dans les champs humides et les alpages; plus rarement dans les bois.

D. h. Depuis Saint-Martin (au-dessus de Luette) jusqu'au haut de la vallée, Evolène, Haudères, Alpe de Veisivi, combes de Ferpècle et d'Arolla.

D. v. Jusqu'à $2000^{\mathrm{m}}$ environ dans le fond de la vallée et $2300^{\mathrm{m}}$ environ à l'Alpe de Veisisi.

$$
\text { Sub. var. ex forma. }
$$

F. intermedia (Moll. Ferret, II, $\mathrm{n}^{\circ} 32$, Remarque).

F. minima. Forèts des Haudères et près de Getty.

$F$. conoidea. Environs des Haudères, etc.

F. depressa. Veisivi, etc.

Gen. Chilotrema Leach.

\section{Chilotrema lapicida (L.).}

Hab. Sous les pierres et le bois mort, dans les vieux murs et contre les rochers. 
D. h. Environs de Bramois, jusqu'à Nax, Vex et aux Mayens de Sion.

D. v. Jusqu'à $1350^{\mathrm{m}}$ environ.

\author{
S.-Fam. Helicidae.
}

Gen. Helix L., s. str.

28. Helix pomatia $\mathrm{L}$.

Hab. Dans les champs, au pied des arbustes et des haies; plus rarement dans les forèts et surtout dans les clairières.

D. h. Environs de Bramois, Vex, Hérémence, Mayens de Sion, Nax, Vernamiège, Euseigne, Evolène, les Haudères jusqu'un peu au-dessus du village, dans les forèts des flancs de Veisivi.

D. v. Jusqu'à environ $1800^{\mathrm{m}}$ au-dessus des Haudères et $1350^{\mathrm{m}}$ à Nax et aux Mayens de Sion.

\title{
Var. gessneri Htm.
}

Au-dessus des Haudères, entre 1500 et $1800^{\mathrm{m}}$ d'altitude.

\section{Gen. Tachea Leach.}

\section{Tachea sylvatica (Drap.).}

Hab. Contre les rochers.

D. h. Environs de Bramois, Ermitage de Longeborgne, Vex, Nax, Euseigne et Hérémence.

D. v. Jusqu'à environ $1300^{\mathrm{m}}$.

$F$. depressa, plus fréquente que le type, sur les rochers de l'Ermitage de Longeborgne.

\section{Tachea nemoralis (L.).}

Hab. Dans les champs et les bois, sous le feuillage mort, etc.

D. h. Comme aux environs d'Aproz, cette espèce ne vit pas 
précisément dans le Val d'Hérens, mais dans toute la vallée du Rhòne, jusqu'à l'embouchure des vallées latérales.

D. v. $490-500^{\mathrm{m}}$ environ.

\section{S.-Fam. Xerophilinae. \\ Gen. Xerophila Held.}

31. Xerophila (Xerophila s. str.) ericetorum (Müll.).

Hab. Dans les champs, au bord des chemins, sur les talus.

D. h. Environs de Bramois, Ermitage de Longeborgne et Nax; environs d'Evolène et de Villa.

D. v. Jusqu'à $1300^{\mathrm{m}}$ à Nax et $1350-1700^{\mathrm{m}}$ près de Villa.

\section{Xerophila (Xerophila) obvia (Htm.).}

Hab. Au bord d'une grand'route, contre des rochers et dans des champs le long des tiges de céréales.

D. h. J'ai observé, le 5 juillet 1912, une petite station d'une cinquantaine d'individus, établis sans doute depuis quelques jours seulement, et tous localisés sur une vingtaine de mètres carrés, entre Euseigne et la Luette. J'en ai rapporté quelques exemplaires aux Mayens de Sion, dans un pré où ils s'acclimateront certainement.

D. v. $980^{\mathrm{m}}$ environ. La station des Mayens de Sion serait à environ $1300^{\mathrm{m}}$.

Remarque. En 1910 j'ai fait quelques racherches le long de cette route, entre Vex et Evolène, sans découvrir une seule X. obvia.

33. Xerophila (Striatella) candidula (Stud.).

Hab. Au bord des chemins, dans les champs, le long des herbes.

D. h. Dans le bas de la vallée, environs de Bramois, de Nax, de Vex et jusqu'au-dessus d'Euseigne:

D. v. Jusqu'à $1000^{\mathrm{m}}$ environ. 


\section{Fam. Buliminidae.}

Gen. Buliminus Ehr.

\section{Buliminus (Zebrina) detritus (Müll.).}

Hab. Dans les champs de céréales, "sur les tiges et les feuilles desquelles il se dissimule, au milieu des ombres portées par celles-ci, comme le tigre au milieu des jungles ${ }^{1}$ ").

D. h. Bramois, Ermitage de Longeborgne, Nax, Vex, Enseigne, Hérémence, Saint-Martin, environs d'Evolène et jusqu'à Villa et à la Forclaz (au-dessus des Haudères).

D. v. Cette espèce atteint $1750^{\mathrm{m}}$ à la Forclaz.

\section{Buliminus (Ena) montanus (Drap.).}

Hab. Sur les vieux troncs et sous le feuillage mort.

D. h. Vex, Mayens de Sion, Hérémence, Suin, Luette et environs des Haudères.

D. v. Jusqu'à environ $1800^{\mathrm{m}}$.

\section{Buliminus (Ena) obscurus (Müll.).}

Hab. Sous le bois et le feuillage morts; sur les vieux troncs et les rochers.

D. h. Disséminée dans le fond de la vallée de Vex jusqu'audessus des Haudères.

D. v. Comme l'espèce précédente, jusqu'à environ $1800^{\mathrm{m}}$.

Gen. Chondrula Beck.

\section{Chondrula quadridens (Müll.).}

Hab. Dans les champs et sous les pierres, sur les talus au bord des chemins.

${ }^{1}$ Godet, Catal. Moll. Neuch,, in Bull. Soc. neuch. sc. nat., XXXIV, p. 111. 
D. h. Environs de Bramoix, Nax, Vernamiège, Vex, SaintMartin, environs d'Evolène, des Haudères et jusqu'au fond de la combe de Ferpècle, où j'ai trouvé une petite forme alpine (forma brevissima).

D. v. Jusqu'à $1870^{\mathrm{m}}$.

\section{Fam. Cochlicopidae.}

\section{Gen. Cochlicopa Risso.}

38. Cochlicopa lubrica (Müll.).

Hab. Dans les champs, sous les pierres et le bois mort; dans les forêts sous les cailloux, le feuillage mort, sur les blocs moussus et les vieux trones.

D. h. Dans le bas de la vallée surtout : environs de Bramois, Ermitage de Longeborgne, Vex, Mayens de Sion et sur les flancs de la montagne de Thyon; la Luette.

D. v. Jusqu’à $1900^{\mathrm{m}}$ environ.

\section{Fam. Pupidae.}

$$
\text { Gen. Pupa Drap. }
$$

\section{Pupa (Torquilla) variabilis Drap.}

Hab. Sous des pierres, au bord de la grand'route.

D. h. Près d'Euseigne.

D. v. Environ $1000^{\mathrm{m}}$.

Remarque. Je n'ai pas trouvé la Pupa secale au Val-d'Hérens; la forme que j'ai indiquée sous ce nom dans le Rameau de Sapin (1911, p. 47) avait été déterminée comme un $P$. secale par M. Godet, qui ne soupçonnait pas que l'espèce méridionale pût se trouver en plein Valais, a $1000^{\mathrm{m}}$ d'altitude. Il est en effet curieux que dans une même vallée alpestre, à $700^{\mathrm{m}}$ de différence, 
on trouve le Vertigo arctica et le Pupa variabilis! Ce dernier a une distribution tout à fait méridionale et ses stations les plus rapprochées, à part Tourbillon, sont Bex, Ollon, Aigle, la Fosse (Charpentier), Martigny, vallon inférieur de la Dranse, entre Brocard et Bovernier (Payot) (max. 600-650 $0^{\mathrm{m}}$ ), Ardon, Morges (Stoll), Perroy, Montreux à Glion et aux Gorges du Chauderon (Andrex), Fouilly, Artagne el Belmont (Jefrreys), Clairvaux (Ogérien), les environs de Genève (Kampunan) et la Savoie : Bonneville (РАуот) et les bords du lac d'Annecy: Duingt, Saint-Jorioz, Bredanaz, Chaparon et au Taillefer (en compagnie de Xerophila striala et carthusiana), Moûlier Coutagne).

\section{Pupa (Modicella) avenarea (Brug.).}

Hab. Contre les rochers.

D. h. Comme l'espèce précédente, dans le bas de la vallée: Vex, etc.

D. v. Jusqu'à $1000^{\mathrm{m}}$ environ.

Gen. Pupilia Leach.

\section{Pupilla muscorum (L.).}

Hab. Sous les pierres, au bord des chemins, dans les champs.

D. h. Dans le bas de la vallée: Nax, Saint-Martin, etc. Cuessin la signale jusqu'à Evolène (1887, p. 261).

D. v. Elle ne dépasse sans doute pas $1300^{\mathrm{m}}$.

\section{Pupilla triplicata (Stud.).}

Hab. Comme l'espèce précédente.

D. h. Dans le bas de la vallée : Bramois, Ermitage de Longeborgne, sur tout le flanc de la colline entre Bramois et Nax, Euseigne et la Luette.

D. v. Jusqu'à $1300^{\mathrm{m}}$. 


\section{Gen. Vertigo Müll.}

\section{Vertigo (Alaea) alpestris (Ald.).}

Hab. Sur les blocs moussus.

D. h. Mayens de Sion, la Luette et sur les flancs de la montagne de Thyon.

D. v. 1000-1950 ${ }^{\mathrm{m}}$ environ.

\section{Vertigo (Alaea) arctica (Wallenb.).}

Hab. Sur un gros bloc erratique, sous la mousse.

D. h. Dans une forèt, en amont des Haudères (à l'entrée du Val d'Arolla, sur la rive droite de la Borgne).

D. v. Environ $1700^{\mathrm{m}}$.

Remarque: La présence, dans les Alpes suisses, de cette espèce arctique habitant la Sibérie, la Russie, le Groenland, le nord de la Scandinavie, les îles Férœer et le Tyrol, est fort intéressante et correspond bien aux aires spécifiques des Patula ruderata et Vertigo alpestris. Je n'ai trouvé que quatre exemplaires de cette espèce rare; ils ont tous été vus et déterminés par M. le Dr Paul Goder, qui en a gardé deux dans sa collection.

\section{Gen. Isthmia Gray.}

\section{Isthmia muscorum (Drap.).}

Hab. Sous les pierres et le bois mort, au bord des chemins, dans les champs.

D. h. Entre Bramois et Nax, sur tout le flanc de la colline.

D. v. $500-1300^{\mathrm{m}}$. 


\section{Fam. Clausiliidae.}

\section{Gen. Balea Prid.}

\section{Balea perversa (L.).}

Hab. Sur les blocs moussus.

D. h. Environs de Bramois, Vex, Mayens de Sion, Vernamiège et jusqu'au fond de la vallée, à Getty et aux Haudères.

D. v. Jusqu'à environ $1800^{\mathrm{m}}$.

\section{Gen. Clausilia Drap.}

\section{Clausilia (Cusmicia) dubia Drap.}

Hab. Sous les pierres et le bois mort; sur les vieux troncs et les blocs moussus.

D. h. Bramois, Nax, Vernamiège, Suin, Mayens de Sion, Hérémence, la Luette, jusqu'au fond de la vallée, au-dessus des Haudères, dans les forêts de Veisivi et des combes d'Arolla et de Ferpècle.

D. v. Environ jusqu'à $1900^{\mathrm{m}}$.

\section{Clausilia (Pirostoma) plicatula Drap.}

Hab. Sur les vieux troncs, sous les pierres et le bois mort.

D. h. Disséminée dans toute la vallée, de Bramois (Longeborgne, etc.) jusqu'aux forêts des Haudères.

D. h. Jusqu'à environ $1800^{\mathrm{m}}$.

\section{Clausilia (Pirostoma) ventricosa Drap.}

Hab. Sur les vieux troncs et les rochers très humides.

D. h. De Bramois à Luette.

D. v. Jusqu'à $1000^{\mathrm{m}}$ environ. 


\section{Fam. Succineidae.}

\section{Gen. Succinea Drap.}

50. Succinea (Amphibina) pfeifferi Rossm.

Hab. Au bord des mares, sous les pierres et le long des herbes.

D. h. Dans la vallée du Rhòne, à l'embouchure du Val d'Hérens et jusqu'aux Mayens de Sion, au-dessus de Vex.

D. v. Jusqu'à $1300^{\mathrm{m}}$.

\section{Succinea (Lucena) oblonga Drap.}

Hab. Dans les prairies très humides, au bord des ruisseaux.

D. h. Je ne l'ai trouvée qu'à Mage; elle est certainement plus largement répandue.

D. v. $1300-1350^{\mathrm{m}}$ environ.

\section{Ord. BASOMMATOPHORA}

\section{Fam. Limnaeidae.}

\section{Gen. Limnaea Lam.}

52. Limnaea (Gulnaria) limosa (L.), sensu latissimo.

$$
\text { Subsp. peregra (Müll.). }
$$

Hab. Dans un fossé.

D. h. Aux Mayens de Sion, au-dessus de Vex.

D. v. Environ $1300^{\mathrm{m}}$. 


\section{Var. cornea (Zgl.).}

Hab. Dans des flaques très peu profondes, au bas des prairies humides.

D. h. Près de Mage. "Coquille plus petite, plus mince, un peu transparente, cornée. » (Moq.-Tand.)

D. v. Environ $1300-1350^{\mathrm{m}}$.

\section{Var. ullepitschi Westerl.}

Hab. Une petite mare assez profonde et vaseuse, remplie de plantes aquatiques, blocs de rochers, et habitée par des Batraciens (Triton alpestris Laur, etc.).

D. h. La Gouille, au-dessus des Haudères.

D. v. $1852^{\text {m }}$.

Remarque : Cette forme est, selon toutes probabilités, la variété de Westerlund ; jai reçu sous ce nom-là une Limnée très voisine recueillie en 1903, à Chamonix, par M. le marquis DE Monterosato.

Elle est de taille plutòt petite, très fragile, très mince, finement striée, transparente, corné plus ou moins pâle. Spire intacte, plutòt allongée, formée de 5-6 tours un peu convexes. Ouverture ovale arrondie, occupant un peu plus de la moitié de la longueur totale, bordée d'un péristome extrèmement mince, tranchant, régulier. Columelle mince, tordue.

La forme fragile et allongée que m'a communiquée M. DE Monterosato est seulement de taille un peu plus petite que mes exemplaires valaisans.

\section{Limnaea (Fossaria) truncatula (Müll.).}

Hab. Dans les flaques, les mares et les "bysses ».

D. h. Environs des Mayens de Sion, au-dessus de Vex; SaintMartin.

D. v. Jusqu'à $1300-1400^{\mathrm{m}}$. 


\section{S.-Cl. PNEUMOPOMA}

Fam. Cyclophoridae.

S.-Fam. Pomatiasinae.

Gen. Pomatias Stud.

54. Pomatias septemspirale (Raz.).

Hab. Sous les pierres et le feuillage mort.

D. h. Je ne l'ai trouvé que dans les forêts des Haudères.

D. v. $1450-1700^{\mathrm{m}}$ environ.

\section{ACEPHALA}

Fam. Sphoeriidae.

Gen. Pisidium C. Pfr.

55. Pisidium (Fossarina) fossarinum Cl.

Hab. Enfoncé dans la vase des mares et des bysses.

D. h. Mayens de Sion et au-dessus de Thyon, sur la crète entre les deux vallées. (Voir Moll. Nendaz, III, $\mathrm{n}^{\circ} 54$.)

D. v. $1300-2200^{\mathrm{m}}$.

Mollusques récoltés aux environs de Loèche-les-Bains, dans la partie supérieure de la vallée de la Dala.

On trouve déjà, à ce sujet, divers renseignements épars dans Studer (Systemat. Verzeichn. der bis jetzt bekannt geword. schweiz. Conchylien); Charpentier (Catalogue des Moll. terrestres et fluv. de la Suisse); Chessin (Die Moll. Fauna Oesterreich- 
Ungarns und der Schweiz); BoLr.rnger (Zur Gastropodenfauna son Basel und Umgebung), et surtout dans les adjonctions manuscrites que Brot a faites en marge de son Catalogue de Charpentier ${ }^{1}$. Je rapporte ici mes observations personnelles faites aux environs de Loèche-les-Bains, malheureusement seulement dans la partie supérieure de la vallée, en les complétant avec ce que j'ai pu trouver dans les auteurs cités. Pour éviter des répétitions, voici la liste de 13 espèces que Вrot a recueillies et que j'ai retrouvées en relevant les conditions biologiques et hypsométriques :

Arianta arbuslorum v. alpicola et albinos.

Tachea sylvatica f. minor.

Helicodonta holoserica et obvoluta.

Euconulus fulvus.

Xerophila ericetorum .

Patula rotundata et ruderata.

Hyalina glabra.

Buliminus obscurus et montanus.

Clausilia ventricosa.

Il n'indique partout comme provenance que Loèche-les-Bains.

\section{GASTROPODA}

\section{S.-Cl. PULMONATA}

\section{Ord. STYLOMMATOPHORA}

\section{Fam. Vitrinidae.}

Gen. Limax Müll.

\section{Limax (Heynemannia) maximus (L.).}

Hab. Sous les pierres et le bois mort, dans les forêts.

${ }^{1}$ VAN den Broeck (1875) donne également divers renseignements sur Loèche et la Gemmi. 
D. h. Commune aux environs de Loèche-les-Bains : forèts des environs d'Albinen, Planetry, d'Allmend, de Tschafenen et de Mascherel.

D. v. Jusqu'à environ $1900^{\mathrm{m}}$.

\section{Limax (Heynemannia) tenellus Nils.}

Hab. Comme l'espèce précédente dans les forèts, ou dans la région alpine, sous les cailloux.

D. h. Forêts de Planetry, d'Allmend et de Mascherel; chemin de la Gemmi.

D. v. Jusqu'à environ $2100^{\mathrm{m}}$.

Gen. Agriolimax Simroth.

3. Agriolimax agrestis (L.).

Hab. Dans les champs et les forèts, sous les pierres, le bois pourri, etc.

D. h. Tout le long de la grand'route jusqu'à Loèche-les-Bains et dans les champs des environs; Albinen, Planetry, Alpe de Clavinen; au-dessus de Loèche sur le Chemin de la Gemmı (Allmend, etc.).

D. v. Elle atteint $2100^{m}$, comme l'espèce précédente.

Gen. Vitrina Drap.

4. Vitrina (Phenacolimax) pellucida (Müll.).

Hab. Sous les pierres et le feuillage mort, dans les forêts.

D. h. Environs de Loèche-les-Bains, forèts d'Allmend et de Planetry.

D. v. Jusqu'à environ $1850^{\mathrm{m}}$.

\section{Vitrina (Semilimax) diaphana Drap.}

Hab. Dans les régions des forêts et alpine, sous les pierres.

D. h. Aux environs de Loèche-les-Bains, en compagnie de 
l'espèce précédente, Allmend, Planetry, forêts de Mascherel et jusqu'à la Gemmi où Bollinger la signale.

D. v. $1300-2300^{m}$ environ.

\section{Gen. Hyalina Fér.}

\section{Hyalina (Euhyalina) glabra (Stud.).}

Hab. Sur des rochers humides ou dans les forêts, sous les pierres et le feuillage mort.

D. h. Le long de la grand'route jusqu'à Loèche-les-Bains; dans les forêts d'Allmend et de Tschafenen.

D. v. Jusqu'à $1700^{\mathrm{m}}$ environ.

\section{Hyalina (Euhyalina) cellaria (Müll.).}

Hab. Cette espèce vit dans les mêmes conditions que la précédente.

D. h. Le long de la grand'route jusqu'à Loèche-les-Bains et dans la forêt d'Allmend.

D. v. Jusqu'à $1700^{\mathrm{m}}$ environ.

\section{Hyalina (Polita) nitens (Mich.).}

Hab. Sous les pierres et le fenillage mort, sur les vieux troncs: dans les forèts.

D. h. Dans toutes les forêts des environs de Loèche-les-Bains : Albinen, Planetry, forêts d'Allmend, de Tschafenen et de Mascherel.

D. v. Jusqu'à $1850^{\mathrm{m}}$ environ.

\section{Hyalina (Polita) pura (Ald.).}

Hab. Dans les forêts, sous les pierres et le feuillage mort.

D. h. Dans les forêts de Planetry, d'Allmend et de Tchafenen.

D. v. Jusqu'à $1850^{\mathrm{m}}$ environ ${ }^{1}$.

${ }^{1}$ Vax dex Broeck (1875) signale à Loèche les Hyalina Draparnaldi (Beck) et nitidula (Drap.), mais sans indications suffisantes. 


\section{Zonitoides nitidus (Müll.).}

Studer signale une petite forme de cette espèce à la Gemmi $\left(2000^{\mathrm{m}}\right.$ environ d'altitude).

\section{Fam. Naninidae.}

Gen. Euconulus Reinh.

\section{Euconulus fulvus (Müll.).}

Hab. Dans les forèts, sous les pierres, le bois et le feuillage mort.

D. h. Forêts d'Albinen, de Planetry, d'Allmend, de Tschafenen et de Mascherel.

D. v. Jusqu'à $1850^{\mathrm{m}}$ environ.

Fam. Arionidae.

Gen. Arion Fér.

\section{Arion empiricorum Fér.}

Hab. Dans les champs et les forêts, sous les pierres, le bois et le feuillage mort.

D. h. Dans tout le fond de la vallée jusqu'à Loèche-les-Bains; dans les forèts des bords de la Dala.

D. v. Jusqu'à $1450^{\mathrm{m}}$ environ.

\section{Arion subfuscus (Drap.).}

Hab. Sous les pierres, dans les forêts, les pâturages et les rocailles de la région alpine.

D. h. Environs de Loèche-les-Bains, forêts d'Allmend, de Tschafenen, Alpe de Clavinen, forêt de Mascherel et chemin de la Gemmi, jusqu'au col.

D. v. $1900^{\mathrm{m}}$ à Clavinen et $2350^{\mathrm{m}}$ à la Gemmi. 
14. Arion hortensis Fér.

Hab. Dans les champs et les forèts, sous les pierres, le bois pourri et le feuillage mort.

D. h. Environs d'Albinen, de Planetry, de Loèche-les-Bains; forèts d'Allmend, de Tschafenen, de Mascherel et sur le chemin de la Gemmi.

D. v. Jusqu'à environ $1900^{\mathrm{m}}$.

\section{Fam. Polyplacognatha.}

Gen. Sphyradium Charp. (West.).

15. Sphyradium gredleri (Cless).

Chessin signale cette espèce à la Gemmi; elle s'y trouve à $2300^{\mathrm{m}}$.

\section{Sphyradium edentulum (Drap.).}

Ввот a recueilli cette espèce dans les bois humides, à Loècheles-Bains, à environ $1500^{\mathrm{m}}$.

\section{Fam. Patulidae.}

\section{Gen. Patula Held.}

\section{Patula (Discus) rotundata (Müll.).}

Hab. Dans les forèts inférieures, sous les pierres et le bois mort; dans les champs, au pied des haies, sous les pierres.

D. h. Dans le fond de la vallée jusqu'à Loèche-les-Bains, Gochti et Buljos.

D. v. Jusqu'à environ $1600^{\mathrm{m}}$.

18. Patula (Discus) ruderata (Stud.).

Hab. Dans les forèts, sous les pierres, le bois mort et sur les vieux troncs. 
D. h. Environs de Loèche-les-Bains, Goppen, Noyer, forèts d'Allmend, de Planetry, d'Albinen, de Tschafenen et de Mascherel.

D. v. $1400-1900^{\mathrm{m}}$ environ.

Gen. Pyramidula Fitz.

19. Pyramidula rupestris (Drap.).

Hab. Contre les rochers et les vieux murs.

D. h. Le long de la grand'route jusqu'à Loèche-les-Bains, chemin des échelles d'Albinen, sur les bords de la Dala et jusqu'à l'Alpe de Clavinen.

D. v. Jusqu'à environ $1900^{\mathrm{m}}$.

\section{Fam. Helicidae.}

S.-Fam. Helicodontinae.

Gen. Helicodonta Risso.

20. Helicodonta (Trigonostoma) obvoluta (Müll.).

Hab. Dans les forèts, sous les pierres et le bois mort.

D. h. Dans le fond de la vallée jusqu'à Loèche-les-Bains; forèt d'Allmend.

D. v. Jusqu'à $1500^{\mathrm{m}}$ environ, c'est-à-dire passablement plus haut que dans les quatre dernières vallées.

\section{Helicodonta (Trigonostoma) holoserica (Stud.).}

Hab. Dans les forèts, sous les pierres et dans de vieux murs, sous la mousse.

D. h. Environs de Loèche-les-Bains, dans les forèts d'Allmend, de Tschafenen et de Mascherel.

D. v. $1400-1900^{\mathrm{m}}$ environ. 


\section{S.-Fam. Fruticicolinae.}

\section{Gen. Fruticicola Held.}

22. Fruticicola (Fruticicola s. str.) sericea (Drap.).

Hab. Sous les pierres et le bois mort.

D. h. Dans le fond de la vallée, aux environs de Loèche-lesBains, d'Albinen, de Planetry et dans les forèts d'Allmend et de Tschafenen.

D. v. Jusqu'à environ $1850^{\mathrm{m}}$.

\section{Fruticicola (Euomphalia) strigella (Drap.).}

Hab. A la lisière des forêts, au pied des haies, sous les pierres et dans de vieux murs.

D. h. Environs de Loèche-les-Bains, Gochti et Buljos.

D. v. Jusqu'à $1600^{\mathrm{m}}$ environ.

\section{S.-Fam. Campylaeinae.}

\section{Gen. Arianta Leach.}

\section{Arianta arbustorum (L.).}

Hab. Dans les champs et plus rarement dans les forêts.

D. h. Jusqu'à proximité de Bürchen, dans le fond de la vallée.

D. v. A peine jusqu'à $1300^{\mathrm{m}}$.

$$
\text { Var. alpicola Charp. }
$$

Hab. Dans les champs, les forêts, les pâturages et les rocailles des régions alpines.

D. h. De Bürchen à Loèche-les-Bains, forêts d'Allmend, de Tschafenen, Alpe de Clavinen, forêt de Mascherel et sur le chemin de la Gemmi, jusqu'au col.

D. v. Jusquà $2330^{\mathrm{m}}$ environ à la Gemmi et $1900-2000^{\mathrm{m}}$ à Clavinen. 
S.-Fam. Helicinae.

Gen. Helix L. s. str.

\section{Helix pomatia L.}

Hab. Dans les champs, au pied des haies, en forêt, dans les clairières; dans les pâturages.

D. h. Très commune dans le fond de la vallée, à Loèche-lesBains, sur le chemin des Echelles d'Albinen, forèts d'Allmend, Planetry, Albinen, Gochti, Dotrenweid et presque jusqu'à Clavinen.

D. v. Jusqu'à environ $1800^{\mathrm{m}}$.

Gen. Tachea Leach.

26. Tachea nemoralis (L.).

Hab. Contre les rochers, au pied des haies et dans les buissons.

D. h. Dans le fond de la vallée, mais moins haut que l'A rianta arbustorum normale.

D. v. Seulement jusqu'à environ $1200^{\mathrm{m}}$.

\section{Tachea sylvatica (Drap.).}

Hab. Contre les rochers, aussi bien le type que la var. montalla.

D. h. Disséminée dans le fond de la vallée, le long de la grand'route, jusqu'au-dessus de Loèche-les-Bains.

D. v. Jusqu'à environ $1500^{\mathrm{m}}$.

Mut. albinos Charp. Assez fréquente.

Var. montana Stud.

D. h. Au-dessus de Loèche-les-Bains, Dotrenweid, Alpe de Clavinen, Mascherel et jusqu'au sommet de la Gemmi.

D. v. Jusqu'à environ $1900^{\mathrm{m}}$ à Clavinen et $2330^{\mathrm{m}}$ à la Gemmi. 
S.-Fam. Xerophilinae.

Gen. Xerophila Held.

28. Xerophila (Xerophila s. str.) ericetorum (Müll.).

Hab. Dans les champs, au bord des chemins, le long des herbes et céréales.

D. h. Disséminée dans le fond de la vallée, Albinen, près de Planetry, Loèche-les-Bains.

D. v. Jusqu’à $1600^{\mathrm{m}}$ environ.

\section{Xerophila (Striatella) candidula (Stud.).}

Hab. Elle se trouve dans les mêmes stations que l'espèce précédente et dans les mèmes conditions biologiques et hypsométriques.

\section{Fam. Buliminidae.}

\section{Gen. Buliminus Ehr.}

30. Buliminus (Zebrina) detritus (Müll.).

Hab. Dans les mèmes conditions biologiques que les Xerophila précédentes.

D. h. Environs de Loèche-les-Bains, Planetry, Albinen, ainsi que sur l'autre rive de la Dala, aux environs de Bürchen.

D. v. Jusqu'à $1600^{\mathrm{m}}$ environ.

\section{Buliminus (Ena) montanus (Drap.).}

Hab. Dans les forèts, sur les vieux troncs et sous le feuillage mort.

D. h. Forèts des bords de la Dala, Loèche-les-Bains, forêts d'Allmend et de Tschafenen.

D. v. Jusqu'à $1850^{\mathrm{m}}$ environ. 


\section{Buliminus (Ena) obscurus (Müll.).}

Hab. Au bord des grand routes, contre les rochers humides, dans les forèts, sur les vieux troncs, sous les pierres, le bois pourri et le feuillage mort.

D. h. Le long de la grand'route jusqu'à Loèche-les-Bains, Albinen, Planetry, forêts d'Allmend et de Tschafenen, Gochti, Dotrenveid, Clavinen, forèt de Mascherel.

D. v. Jusqu'à environ $1900^{\mathrm{m}}$.

Gen. Chondrula Beck.

33. Chondrula quadridens (Müll.).

Hab. Dans les champs, au bord des chenins, au pied des haies, sous les pierres.

D. h. Le long de la grand'route, environs de Bürchen, Loècheles-Bains; près de Planetry et d'Albinen.

D. v. Jusqu'à $1600^{\mathrm{m}}$ environ.

Gen. Acanthinula Beck.

34. Acanthinula aculeata (Müll.).

Ввот la signale dans les forêts des environs de Loèche-lesBains $\left(1500^{\mathrm{m}}\right.$ environ $)$; rare.

Fam. Cochlicopidae.

Gen. Cochlicopa Risso.

35. Cochlicopa lubrica (Müll.).

Hab. Dans les champs et les forèts, sous les pierres et le bois mort. 
D. h. Environs d'Albinen, Planetry, forèts d'Allmend et de Tschafenen, Loèche-les-Bains.

D. v. Jusqu'à $1850^{\mathrm{m}}$ environ.

\section{Fam. Pupidae.}

Gen. Pupa Drap.

36. Pupa (Torquilla) secale Drap.

Hab. Sous les pierres et le bois mort, au bord des routes et dans les forèts.

D. h. Le long de la grand'route, jusqu'à Loèche-les-Bains, Planetry, forèts d'Allmend et de Tschafenen.

D. v. Jusqu'à $1850^{\mathrm{m}}$ environ.

\section{Pupa (Modicella) avenacea (Brug.).}

Hab. Contre les rochers.

D. h. Le long de la grand'route jusqu'à Loèche-les-Bains, chemin des Echelles d'Albinen, Dotrenweid et jusqu'au-dessus de Clavinen.

D. v. Jusqu'à $1900^{\mathrm{m}}$ environ '.

\section{Fam. Glausiliidae.}

Gen. Balea Prid.

38. Balea perversa (L.).

Hab. Dans les forèts, sur les blocs moussus.

D. h. Environs de Loèche-les-Bains, forèts d'Allmend et de Tschafenen, Planetry.

D. v. Jusqu'à $1850^{\mathrm{m}}$ environ.

${ }^{1}$ Studer (1820) mentionne le Pupilla muscorum (L.) à Loèche-les-Bains, mais on ne sait à quelle altitude. 


\section{Gen. Clausilia Drap.}

\section{Clausilia (Clausiliastra) laminata (Mtg.).}

Hab. Dans les forèts, sur les vieux troncs et sous les pierres.

D. h. Environs de Loèche-les-Bains, forèts de Mascherel, Tschafenen, d'Allmend, chemin des Echelles d'Albinen, Planetry.

D. v. Jusqu'à $1850^{\mathrm{m}}$ environ.

40. Clausilia (Cusmicia) parvula Stud.

Hab. Contre les rochers.

D. h. Le long de la grand'route jusqu'à Loèche-les-Bains, forèt d'Allmend, sur le chemin des Echelles, Gochti et Buljos.

D. v. Jusqu'à environ $1500^{\mathrm{m}}$.

\section{Clausilia (Cusmicia) dubia Drap.}

Hab. Dans les forèts, sous les pierres, les écorces, sur les vieux troncs, les blocs moussus.

D. h. Forèts des bords de la Dala, d'Allmend, de Planetry et de Tschafenen.

D. v. Jusqu'à environ $1850^{\mathrm{m}}$.

Var. obsoleta A. Schm.

D. h. et v. En compagnie du type, dans toutes les forèts.

\section{Clausilia (Cusmicia) cruciata Stud.}

Je n'ai pas trouvé le type de l'espèce; Charpentier (Cat., p. 17, $\mathbf{n}^{\circ} 84$ ) signale la $C$. cruciata sur les rochers calcaires des bains de Loèche. Sans doute s'agit-il de la variété suivante: 
Var. alpestris Stoll.

Hab. Sur les vieux troncs moussus, sous l'écorce.

D. h. Dans la forêt d'Allmend et sur le chemin des Echelles d'Albinen.

D. v. Environ jusqu'à $1700^{\mathrm{m}}$. Je ne l'ai pas trouvée au-dessous de $1400^{\mathrm{m}}$.

\section{Clausilia (Pirostoma) plicatula Drap.}

Hab. Dans les forêts, en compagnie des espèces précédentes.

D h. Forêts des bords de la Dala, d'Allmend et de Tschafenen.

D. v. Jusqu'à $1700^{\mathrm{m}}$ environ.

\section{Clausilia (Pirostoma) ventricosa Drap.}

Hab. Contre les rochers humides.

D. h. Le long de la route jusqu'à Loèche-les-Bains et même au-dessus.

D. v. Jusqu'à environ $1500^{\mathrm{m}}$.

\section{Ord. BASOMMATOPHORA}

Fam. Limnaeidae.

Gen. Limnaea Lam.

45. Limnaea (Gulnaria) limosa (L.) sensu latissimo.

Subsp. peregra (Müll.).

Ввот a recueilli cette sous-espèce dans une source chaude à Loèche-les-Bains. Environ $1400^{\mathrm{m}}$. 
Résumé et conclusions des observations hypsométriques.

Les régions qu'indique Chessin ${ }^{1}$, d'après Heller, né sont pas entièrement satisfaisantes au point de vue malacologique. D'une part, la région des vallées comprend un grand nombre d'espèces montant jusqu'à 1500-1600 ${ }^{\mathrm{m}}$ environ, et, par conséquent, est trop vaste pour n'ètre pas subdivisée; mais surtout. ces régions sont trop absolument délimitées et superposées, de telle sorte qu'il n'y a aucun lien entre la région des vallées et la région alpine, ou entre la région supérieure des forêts et la région subnivale, pour les espèces qui n'habitent pas les forêts ou qui passent directement de la limite supérieure des forèts dans la région subnivale. J'emploie donc une classification un peu plus large, sans superposer nécessairement les trois dernières régions de Heller aux régions des forêts et en admettant deux régions des vallées, l'inférieure qui correspondrait dans une certaine mesure à la "Thalregion " typique, et la supérieure s'avançant dans le fond des vallées latérales, souvent jusqu'à la région alpine.

\section{RÉGion inférieure des Vallées.}

Cette région comprend une partie du Plateau suisse, le fond des grandes vallées (vallée du Rhòne) et la fin des vallées latérales. Dans le fond de ces dernières, cette région ne s'avance guère au-dessus de $1000-1200^{\mathrm{m}}$ à cause de la proximité des hautes montagnes et des glaciers, tandis qu'aux points de jonction entre les vallées latérales et celle du Rhòne, elle atteint facilement $1300^{\mathrm{m}}$ et mème plus, par exemple près de HauteNendaz, Cerisier, la Crêta, à Nax et au-dessus de Vex, aux Mayens de Sion, etc.

1 Die Mollushenfauna Oesterreich-Lngarns und der Schweiz, 1887, p. 8.

Rev. Suisse de Zool. T. 21. 1913. 
Cette région est caractérisée par :

Arion empiricorum ${ }^{1}$.

Vallonia pulchella.

Tachea nemoralis.

Xerophila obvia ${ }^{3}$.

Pupilla muscorum.

Isthmia muscorum.

Limnaea stagnalis.

Limnaea palustris.

Planorbis contortus.

Planorbis complanatus.

Pisidium oblusale.
Arianta arbustorum normale. Tachea sylvatica normale ${ }^{2}$. Xerophila candidula et var. gratiosa.

Pupilla triplicata.

Succinea oblonga ${ }^{4}$.

Succinea pfeifferi.

Physa hypnorum.

Planorbis marginatus.

Planorbis crista.

Valvata cristata.

Eulota fruticum normale.

Le Val Ferret présente en outre une particularité intéressante (voir plus loin la partie spéciale).

\section{Région inférieure des forèts.}

Cette région comprend toutes les forèts inférieures, mais en général elle se conforme moins que la région précédente à la loi de l'enver's et de l'endroit : souvent elle atteint dans le fond des vallées latérales la mème altitude que sur les flancs des montagnes, à la jonction avec la vallée du Rhòne, car les forèts ressentent bien moins l'effet du soleil et de la chaleur que la région dépourvue de leur végétation. C'est ainsi qu'à Loècheles-Bains, l'Helicodonta obvoluta atteint $1500^{\mathrm{m}}$, alors que près

${ }^{1}$ Celte espèce fait exception dans le Val d'Hérens et à Loèche-les-Bains, où elle atteint $1450^{\mathrm{m}}$.

2Malgré son nom, je n'ai trouvé, au Valais, le type de celte espèce que sur des rochers, en dehors des forèts.

s Cependant une colonie de $X$. obvia s'est élablie à Ia Seilo à $1500 \mathrm{~m}$; la faible altitude que cette espèce atteint actuellement n'est sans doute que provisoire, tant que l'invasion est ì ses débuts.

4 Je n'ai pas trouvé cette espèce au Valais à frlus de $1350^{\mathrm{m}}$, mais elle atteint $1600^{\mathrm{m}}$ au Bündnerland et mème $1950^{\mathrm{m}}$ au Mont-Cenis (Koвelt). 
de Haute-Nendaz (forèts de Praz-Condjuz) et aux Mayens de Sion, c'est-à-dire sur les bords de la vallée du Rhòne, cette espèce ne monte qu'à $1350^{\mathrm{m}}$. D'autre part, dans le fond du Val d'Hérens, à Luette, on trouve l'Helicodonta holoserica, espèce caractéristique de la région suivante, à $1000^{\mathrm{m}}$ d'altitude.

Ces différences entre les limites supérieures de la région inférieure des forèts de la vallée de Loèche et du Val d'Hérens s'expliquent très bien par la dissemblance des conditions climatologiques résultant de la situation septentrionale ou méridionale des vallées latérales par rapport au Rhòne. Un exemple de la mème loi, dans la région précédente, est la Tachea nemoralis, que je n'ai pas trouvée au-dessus de $500^{\mathrm{m}}$ au sud du Rhòne, alors qu'au nord, à l'embouchure des vallées latérales mieux exposées, celte espèce atteint environ $1000^{\mathrm{m}}$ dans le vallon de l'Avançon et $1200^{\mathrm{m}}$ dans la vallée de Loèche. Comme on le voit, il y a un étroit rapport entre les régions inférieures des vallées et des forèts, et j'ai hésité à les distinguer. Mais cependant il y a un certain nombre d'espèces caractéristiques pour chacune d'elles, et surtout il y a un grand nombre de Nollusques habitant soit les deux régions des vallées, soit les deux régions des forèts. En outre, comme je viens de le dire, il y a des différences entre les distributions hypsométriques des espèces des forèts et de celles des champs, et la plupart des espèces sont nettement sylvatiques (hygrophiles) ou xérophiles, cette distinction étant assez douteuse à de basses altitudes, mais devenant plus absolue à mesure que l'on s'élève.

Voici les espèces caractériques de cette région :

Hyalina villae.

Patula rotundata ${ }^{1}$.

Fruticicola incarnata.
Fruticicola strigella ${ }^{2}$. Isognomostoma personatum ${ }^{3}$. Acanthinula aculeata.

1 Au Val Ferret, cette espèce fait une grande exception avec $1800 \mathrm{~m}$.

${ }^{2}$ Cette espèce, ne dépassant guère $1000^{\mathrm{m}}$ dans le Jura et pas $1350^{\mathrm{m}}$ dans les vallées des Plans, de Ferret et de Nendaz, semble bien caractéristique, mais elle atteint $1700^{\mathrm{m}}$ à la Forclaz, an Val d'Hérens.

${ }^{3}$ Cette espèce semble assez caractéristique, mais atteint cependant le commencement de la région suivante, jusqu'à environ $1600^{\mathrm{m}}$. 
Clausilia parvula. Sphyradium edentulum. Helicodonta obvoluta. Fruticicola villosa.
Chilotrema lapicida.

Tachea hortensis.

Vertigo pygmaea ${ }^{1}$.

Clausilia ventricosa .

\section{II. Région supérieure des forêts.}

Elle comprend tout le reste des forêts, généralement à partir d'environ $1200-1350^{\mathrm{m}}$ (parfois plus bas, dans le fond des vallées méridionales ou beaucoup plus haut, dans les vallées septentrionales), jusqu'à la limite supérieure des forêts, c'est-à-dire à une altitude variant suivant les stations entre 1800 et $2200^{\mathrm{m}}$. Autrement dit, sa limite inférieure est absolument en fonction de la région précédente; il en est de même pour la région supérieure des vallées.

A partir d'environ $1400-1500^{\mathrm{m}}$, les régions supérieures des forêts et des vallées sont tout à fait distinctes l'une de l'autre; elles n'ont plus de commun que les Arion subfuscus et hortensis, Euconulus fulvus, Helix pomatia, Arianta arbustorum var. alpicola, Tachea sylvatica var. montana, Pyramidula rupestris et Cochlicopa lubrica, qui ont une distribution hypsométrique encore beaucoup plus vaste.

Voici les espèces caractéristiques:

Vitrina elongata var. sapinea. Hyalina depressa.

Hyalina nitens var. dutail- Hyalina radiatula var. petrolyana.

Patula ruderata. nella.

Vertigo alpestris.

Balea perversa var. vitrina.

Clausilia fimbriala.

Helicodonta holoserica.

Vertigo arclica.

Clausilia cruciata et var. alpestris.

\footnotetext{
${ }^{1}$ En contradiction avec les données de Chessix et de Bollinger, je n'ai pas rencontré cette espèce au-dessus de $1300^{\mathrm{m}}$.

2 Cette espèce semble assez caractéristique, mais atteint cependant le commencement de la région suivante, jusquà environ $1600^{\mathrm{m}}$.
} 
En outre, les Vitrina diaphana et Sphyradium gredleri sont assez caractéristiques, mais se retrouvent plus haut (région alpine). Dans le Val Ferret, les forèts se sont établies sur d'anciennes moraines dans lesquelles est demeurée la Campyloea foetens, à la plaine de Saleinaz et à la Neuva.

\section{Région Supérieure des Vallées.}

Cette région, ne comprenant ordinairement que des terrains d'alluvions, occupe le fond des vallées latérales, d'environ $1000-1200^{\mathrm{m}}$ jusqu'aux forèts qui la séparent le plus souvent des pâturages supérieurs de la région alpine. Il est à remarquer que ces deux régions sont bien moins caractérisées par les espèces qui y habitent que par l'absence d'autres espèces. Au Val Ferret, la région supérieure des vallées est déterminée par la présence de la var. godeliana de l'Eulota fruticum; cependant, dans les autres vallées, malgré l'absence de formes spéciales à cette région, on la distingue très facilement de la région inférieure des vallées: $1^{\circ}$ par l'apparition de l'Arianta arbustorum var. alpicola et de la Tachea sylvatica var. montana, deux formes communes à toutes les régions supérieures; et $2^{\circ}$ par la disparition du type de ces deux espèces et des formes caractéristiques des altitudes inférieures, indiquées au $\S 1$. D'autre part, plusieurs espèces sont communes aux deux régions des vallées: les Xerophila ericetorum et candidula, Buliminus detritus, Chondrula quadridens (pas partout, souvent spéciale à la région inférieure: Val Ferret, etc.), etc.

\section{Région alpine.}

Cette région comprend tous les pâturages et alpages très élevés, au-dessus de la limite supérieure des forèts, ainsi que les rocailles des crètes et sommets peu élevés $\left(2300^{\mathrm{m}}-2800^{\mathrm{m}}\right.$ environ) et éloignés de toute neige éternelle et de tout glacier (Alpe de Veisivi au pied des dents de Veisivi, Gemmi, Tour de Bavon, Dent de Nendaz, etc.). En Valais, on ne peut la caracté- 
riser que par la Pupilla alpicola, mais un certain nombre d'autres espèces atteignent des altitudes très grandes relativement à l'eukoumène hypsométrique des Mollusques:

1. Agriolimax agrestris : Gemmi $2100^{\mathrm{m}}$, Tortin $2200^{\mathrm{m}}$.

2. Limax tenellus: Gemmi $2100^{\mathrm{m}}$.

3. Vilıina pellucida var. alpina: Tour de Bavon $2481^{m}$, GrandDésert $2900^{\mathrm{m}}$.

4. Vitrina diaphana: Tour de Bavon $2481^{\mathrm{m}}$, Gemmi $2300^{\mathrm{m}}$.

5. Euconulus fulous : Dent de Nendaz $2467^{\mathrm{m}}$, Tour de Bavon $2481^{m}$.

6. Zoniloides nitidus : Gemmi $2000^{\mathrm{m}}$.

7. Arion subfuscus : Tête-Moutse $2000^{\mathrm{m}}$, Saleinaz $2200^{\mathrm{m}}$, Arolla $2300^{\mathrm{m}}$, Gemmi $2300^{\mathrm{m}}$, Tortin $2350^{\mathrm{m}}$, Dent de Nendaz $2467^{\mathrm{m}}$, Tour de Bavon $2481^{\mathrm{m}}$, Mont-Gelé $2735^{\mathrm{m}}$.

8. Punctum pygmæum : Dent de Nendaz $2467^{m}$, Tour de Bavon $2481^{\mathrm{m}}$.

9. Pyramidula rupesiris : Dent de Nendaz $2467^{\mathrm{m}}$, Tour de Bavon $2481^{\mathrm{m}}$, Mont-Gelé $2735^{\mathrm{m}}$ et Grand-Désert $2800-2900^{\mathrm{m}}$.

10. Fruticicola sericea: Tour de Bavon $2481^{\mathrm{m}}$.

11. Arianta arbustorum var. alpicola: Clavinen $2000^{\mathrm{m}}$, TèteMoutse $2100^{\mathrm{m}}$, Thyon $2200^{\mathrm{m}}$, Alpe de Veisivi $2300^{\mathrm{m}}$, Gemmi $2330^{\mathrm{m}}$, Dent de Nendaz $2467^{\mathrm{m}}$, Tour de Bavon $2481^{\mathrm{m}}$.

12. Tachea sylvatica var. montana: Gemmi $2330^{\mathrm{m}}$, Dent de Nendaz $2467^{\mathrm{m}}$, Tour de Bavon $2481^{\mathrm{m}}$.

13. Cochlicopa lubrica var. minima: Tour de Bavon $2481^{\mathrm{m}}$.

i4. Vertigo alpestris: Dent de Nendaz $2467^{\mathrm{m}}$.

15. Pupa secale var. minor: Tour de Bavon $2481^{\mathrm{m}}$.

16. Pupilla alpicola (spécial à la région): Tour de Bavon $2481^{\mathrm{m}}$; var. saxetana (id.) : Lac des Veaus $2550^{\mathrm{m}}$, Mont-Gelé $2735^{\mathrm{m}}$.

17. Clausilia dubia var. alpicola: Dent de Nendaz $2467^{\mathrm{m}}$.

18. Limnaea limosa subsp. peregra: Col des Etablons $2150^{\mathrm{m}}$.

19. Limnaea truncatula: Sept-Fontaines $2100^{\mathrm{m}}$, Bavon $2200^{\mathrm{m}}$.

20. Pisidium fossarinum: Cleuson $2100^{\mathrm{m}}$, Col des Etablons $2150^{\mathrm{m}}$, Lac-Noir $2200^{\mathrm{m}}$, Bavon $2200^{\mathrm{m}}$; var. major : Bavon $2200^{\mathrm{m}}$. 


\section{Vi. Région subivale.}

Cette région est caractérisée par la présence immédiate des glaciers et des neiges; elle est formée de rocailles, d'éboulis et de moraines couverts d'une petite végétation alpestre (Artemisia glacialis et mutellina, Linaria alpina, Ranunculus glacialis), ou presque sans aucune végétation, au milieu de taches de neiges éternelles, comme à la cabane de Saleinaz. Cette région ne se trouve pas nécessairement au-dessus de la précédente; par exemple, près du glacier de Saleinaz il y a de grandes étendues de moraines apparlenant d'une manière bien caractéristique à la région subnivale, entre 1350 et $1500^{\mathrm{m}}$, alors que les flancs des montagnes avoisinantes (par exemple la TêteMoutse) ne peuvent se ranger que dans la région alpine $1400-$ $2100^{\mathrm{m}}$ : Arianla arbustorum var. alpicola au milieu des Rhododendrons). De même, entre le Mont-Gond et le Mont-Gelé, les deux régions alpine et subnivale sont bien près de se confondre; on trouve dans chacune des enclaves de l'autre : tantòt des alpages parsemés de quelques rocailles (Pupilla alpicola, Pyramidula rupestris, Arion subfuscus), tantòt des pentes d'éboulis formés de rocailles schisteuses et parsemés de grandes plaques de neige qui persistent en général tout l'été il y en avait un grand nombre le 9 aoùt 1912, où l'on trouve l'espèce caractéristique de la région subnivale, la Vitrina nivalis, en compagnie d'Arion subfuscus. Il en est de mème à la Dent de Nendaz, dont toute la partie supérieure fait partie de la région alpine, sauf une enclave située dans une combe ordinairement neigeuse (14 aoùt 1912), où vit la Vitrina nivalis.

Voici les espèces trouvées dans la région subnivale:

1. Vitrina nivalis (spécial à la région): Dent de Nendaz 2350$2460^{\mathrm{m}}$, Mont-Gelé 2600-2800 ${ }^{\mathrm{m}}$, cabane de Saleinaz environ 2700 .

2. Vitrina pellucida var. alpina: Grand-Désert (dE Darded leg.), à la fois dans les régions alpine et subnivale qui s'enchevêtrent l'une dans l'autre, entre 2800 et $2900^{\mathrm{m}}$. 
3. Campylaea foetens: Glacier de Saleinaz $1350-1500^{\mathrm{m}}$, la Neuva $1400-1900^{\mathrm{m}}$ environ, sur les bords du glacier de Saleinaz à $2200^{\mathrm{m}}$, à la cabane à $2700^{\mathrm{m}}$.

4. Arion subfuscus : Glacier de Saleinaz 1350-2400m, Gemmi 2300-2350 ${ }^{\mathrm{m}}$, Dent de Nendaz 2350-2467 ${ }^{\mathrm{m}}$, Mont-Gelé jusqu'à environ $2735^{\mathrm{m}}$.

En outre, sous le glacier de Saleinaz, entre 1350 et $1500^{\mathrm{m}}$ environ, près du torrent qui en sort, au milieu de moraines et sous de gros blocs granitiques, à une température toujours très basse, j’ai trouvé en compagnie de Campylaea foetens les quelques espèces suivantes:

5. Limax maximus.

7. Agriolimax agrestis.

9. Euconulus fulous.

11. Patula ruderata.
6. Limax tenellus.

8. Vilrina diaphana.

10. Arion subfiuscus.

12. Helicorlonia holoserica.

ViI. Région nivale.

Impropre à la vie des Mollusques: aneukoumène malacologique en altitude, commençant à environ $2700-3000^{\mathrm{m}}$, le plus souvent à $2800^{\mathrm{m}}$.

a) Vallon des Plans.

I. Région inférieure des Vallées.

Elle comprend les environs de Frénières, des Vénéresses et des Plans, jusqu'à environ $1150-1200^{\mathrm{m}}$.

Agriolimax agrestis.

Arion subfuscus.

Pyramidula rupestris.

Arianta arbustorum.

Tachea nemoralis.

Pupa avenacea.

Succinea pfeifferi.
Arion empiricorum.

Arion hortensis.

Eulota fruticum.

Helix pomatia.

Xerophila ericetorum.

Chondrula quadridens. 
II. RÉgion inférieure des Foréts.

Toutes les forèts des Gorges de l'Avançon, jusqu'un peu audessus des Plans, à 1200-1250 $\mathrm{m}$ environ.

Limax maximus.

L. maximus var. cinereo niger. Agriolimax agrestis. Hyalina glabra. Hyalina nitens. Hyalina radiatula. Euconulus fulvus. Arion subfuscus. Pyramidula rupestris. Helicodonta obvoluta. Fruticicola villosa. Arianta arbuslorum. Isognomostoma personatum. Tachea hortensis. Buliminus montanus. Cochlicopa lubrica. Balea perversa. Clausilia dubia. Clausilia plicatula. Pomatias septemspirale.
L. maximus var. cinerea.

Limax tenellus. Vitrina diaphana. Hyalina villae. Hyalina pura. Crystallus diaphanus. Arion empiricorum. Arion hortensis. Patula rotundata. Frulicicola sericea. Fruticicola incarnata. Chilotrema lapicida. Helix pomalia. Tachea sylvatica. Buliminus obscur'us. Pupa avenacea. Clausilia laminata. Clausilia parvula. Clausilia ventricosa.

\section{Région supérieure des foréts.}

Au-dessus des Plans, depuis $1200^{\mathrm{m}}$ environ, et aux environs de Pont-de-Nant.

Limax maximus.

Limax tenellus.

Vitrina diaphana.

Hyalina nilens.
Hyalina radiatula.

Euconulus fulvus.

Arion hortensis.

Patula ruderata. 
Frulicicola sericea.

Tachea sylvatica var. montana.

Buliminus montanus.

Cochlicopa lubrica.

Vertigo alpestris.

Clausilia fimbriata.

Clausilia plicatula.

Clausilia dubia.

L. maximus var. cinereo niger.

Agriolimax agrestis.

Hyalina glabra.

Hyalina pura.
Crystallus diaphanus.

Arion subfuscus.

Pyramidula rupestris.

Helicodonta holoserica.

Isognomostoma personatum

(in parte).

Arianta arbustorum var. alpicola.

Buliminus obscurus.

Pupa avenacea.

Clausilia laminata.

\section{Région supérieure des vallées.}

Environs de Pont-de-Nant et du côté des Parts (au-dessus des Plans).

Agriolimax agrestis.

Arion subfuscus.

Arianta arbustorum var. alpicola.

Tachea sylvatica var. montana.

Chondrula quadridens.
Arion hortensis.

Pyramidula rupestris.

Helix pomatia.

Xerophila ericetorum.

Cochlicopa lubrica.

b) Val Ferret.

\section{Région inférieure des vallées.}

Cette région occupe tout le fond de la vallée, depuis Orsières jusqu'un peu au-dessus de Praz-de-Fort, au bas de la plaine de Saleinaz, à $1200^{\mathrm{m}}$ d'altitude.

Du còté de Champex et de la Deuva, au-dessus de Som-la-Proz et de Ville-d'Issert, on constate un phénomène intéressant, déjà relevé en géographie économique: dans toute vallée latérale, comme dans le Val Ferret où cette particularité est très accen- 
tuée, on distingue plus ou moins clairement l'envers ou versant de l'est, privé de soleil le matin et une grande partie de l'aprèsmidi, et l'endroil ou versant de l'ouest, exposé au soleil presque toute la journée et surtout pendant tout le matin. Il résulte de ces conditions climatologiques que, dans cértaines vallées, on trouve à lătitude et à altitude égales une agriculture prospère, des céréales et même des plantes du Midi, à l'endroit, tandis qu'à l'envers la végétation est encore sauvage, la température est beaucoup plus froide et les forêts alpines remplacent les cultures de l'endroit. Il en est ainsi à la Deuva, où ce phénomène permet encore la culture de céréales et influence la répartition altitudinaire des Mollusques, de sorte que la région inférieure des vallées y atteint une altitude passablement plus considérable que dans le reste de la vallée et que dans les autres vallées situées au sud du Rhòne, c'est-à-dire jusqu'à $1450^{\mathrm{m}}$ environ. Il y a quelque chose de semblable dans la distribution de la Hyalina glabra au Val de Nendaz.

Agriolimax agrestis.

Arion empiricorum.

Pyramidula rupestris.

Vallonia helvetica.

Arianta arbustorum normale.

Tachea sylvatica normale.

Xerophila obvia.

$X$. candidula var. gratiosa.

Cochlicopa lubrica.

Pupa avenacea.

Succinea pfeifferi.

Limnaea truncatula.

Pisidium fossarinum v. major.
Hyalina cellaria.

Arion hortensis.

Eulota fiuticum normale.

Fruticicola strigella.

Helix pomatia.

Xerophila ericetorum.

Xerophila candidula.

Buliminus detritus.

Chondrula quadridens.

Pupilla muscorum.

Limnaea limosa subsp. peregra et var.

Pisidium fossarinum.

II. Région inférieure des foréts.

Toutes les forèts jusqu'à l'altitude d'environ 1200-1300"', c'est-à-dire un peu au-dessus de Praz-de-Fort, jusqu'aux forèts 
du Jurassa, de Froumion et de l'Avary, qui forment une zone neutre où les espèces des deux régions des forèts vivent ensemble sur un espace de presque $100^{\mathrm{m}}$ en altitude.

Limax maximus.

L. maximus var. cinereo niger. Agriolimax agrestis.

Vitrina diaphana.

Hyalina nitens.

Hyalina radiatula.

Euconulus fulous.

Arion subfuscus.

Punctum pygmaeum.

Pyramidula rupestris.

Fruticicola edentula.

Fruticicola strigella.

Arianta arbustorum.

Buliminus montanus.

Pupa secale.

Vertigo pygmaea.

Clausilia laminata.

Clausilia plicatula.

Clausilia ventricosa.
L. maximus var. cinerea.

Limax lenellus.

Vitrina pellucida.

IIyalina glabra.

Hyalina pura.

Crystallus crystallinus var. eburnea.

Arion empiricorum.

Arion hortensis.

Patula rolundala.

Helicodonta obvoluta.

Fruticicola sericea.

Chilotrema lapicida.

Isognomostoma personatum.

Buliminus obscurus.

Pupa avenacea.

Balea perversa.

Clausilia dubia.

La C'lausilia paroula trouvée à $1350^{* \prime \prime}$ semble donc influencée par la loi de l'endroit; cependant, avant de conclure, il serait bon de découvrir d'autres stations de cette espèce au Val Ferret.

III. Région supérieure des foréts.

Cette région, d'abord confondue avec la précédente entre 1200 et $1300^{\mathrm{m}}$, occupe d'une manière caractéristique le reste des forêts jusqu'à leur limite supérieure.

Limax maximus.

Limax tenellus.

Vilrina pellucida.
Hyalina glabia.

Hyalina nitens.

Hyalina pura. 
Crystallus cryslallinus var. eburnea.

Arion subfuscus.

Spleyradium gredleri.

Palula rotundata ${ }^{1}$.

Pyramidula rupestris.

Fruticicola sericea.

Campylaea foetens ${ }^{2}$.

Isognomostoma personatum.

Helix pomatia var. parva.

Buliminus montanus.

Cochlicopa lubrica.

Pupa avenacea.

Balea perversa.

Clausilia laminala.

Clausilia cruciala var. alpestris.

L. maximus var. cinereo niger. Agriolimax agrestis.

Vitrina diaphana.
Hyalina depressa.

$H$. nitens var. dutaillyana.

Hyalina radiatula var. petronella.

Euconulus fulvus.

Arion hortensis .

Punctum pygmaeum.

Patula ruderala.

Helicodonta holoserica.

Fruticicola edentula.

Arianta arbustorum var. alpicola.

Tachea sylvatica var. montana.

Buliminus obscurus.

Pupa secale.

Verligo alpestris.

$B$. perversa var. vitrina.

Clausilia dubia.

Clausilia ventricosa.

La Chilotrema lapicida, espèce appartenant à la région inférieure des forêts, atteint pourlant $1400^{\mathrm{m}}$ au-dessus de Prazde-Fort, à une altitude où elle est perdue au milieu d'espèces alpines (Helicodonta holoserica et autres). Un certain nombre d'espèces à distribution intermédiaire n'occupent qu'une partie de la région supérieure des forêts, n'atteignant que $1600^{\mathrm{m}}$ (à la Neuva). Ce sont : Crystallus crystallinus var. eburnea, Fruticicola edentula, Isognomosloma personatum, Clausilia laminala et Clausilia ventricosa. D'autre part, deux espèces, les Hyalina depressa et Clausilia cruciala (var. alpestris), caractéristiques de cette région, ne se rencontrent qu'entre 1200 et $1600^{\mathrm{m}}$ environ. Il en est de mème pour le sphyradium gredleri, que je n'ai trouvé qu'à $1300^{\mathrm{m}}$.

${ }^{1}$ A Ferret, $1800 \mathrm{~m}$.

Voir plus haut: Région supérieure des forêts (généralités). 


\section{Région supérieure des vallées.}

Cette région occupe le fond de la vallée, à partir de la plaine de Saleinaz jusqu'à Ferret $\left(1200-1850^{\mathrm{m}}\right.$ environ. Lac Champex et marais d'alentour.

Agriolimax agrestis.

Arion subfuscus.

Eulota fruticum var. godetiana.

Helix pomalia.

Xerophila obvia.

Cochlicopa lubrica var.minima.

Limnaea limosa subps.peregra.
Arion hortensis.

Pyramidula rupestris.

Arianta arbustorum var. alpicola.

Tachea sylvatica var. montana.

Xerophila ericetorum.

Pisidium fossarinum.

La Xerophila obvia, tenue jusqu'à présent pour une espèce spéciale à la région inférieure des vallées, paraît devoir s'acclimater dans la région supérieure nouvelle station à la Seilo, à $\left.1500^{\mathrm{m}}\right)$.

\section{Région alpine.}

Au-dessus de la limite supérieure des forèts, à partir de $2000^{\mathrm{m}}$ environ: Tète-Moutse, en montant à la cabane de Saleinaz, Bavon, la Crète et jusqu'au sommet de la Tour :

Vitrina pellucida var. alpina. Vilrina diaphana.

Euconulus fulvus.

Arion hortensis ${ }^{1}$.

Pyramidula rupestris.

Arion subfuscus.

Punctum pygmaeum.

Arianta arbustorum var. alpicola.

Cochlicopa lubrica var. minima.

Pupilla alpicola.

Fruticicola sericea.

Tachea sylvatica var. montana. Pupa secale var. minor.

Pisidium fossarinum.

Pisidium fossarinum v. major. Limnaea truncatula.

${ }^{1}$ Cette espèce doit-elle être considérée comme habitaut la région alpine? Elle atteint $2000^{\mathrm{m}}$ à Saleinaz. 


\section{Vi. Région subnivale.}

Elle occupe les environs du glacier de Saleinaz et du glacier de la Neuva, au-dessous desquels j'ai trouvé un certain nombre d'espèces insolites dont la liste est donnée plus haut généralités sur la région subnivale). Dans la région subnivale proprement dite (cabane de Saleinaz : environ $2700^{\mathrm{m}}$ ) je n'ai recueilli que les Vitrina nivalis et Campylaea foetens. Quant à l'Arion subfuscus, il vit au-dessus du glacier entre 2000 et $2400^{\mathrm{m}}$, soit dans la région alpine, soit dans celle-ci.

c) Val de Nendaz.

\section{Région inférieure des Vallées.}

Cette région comprend la fin du Val de Nendaz, s'étendant à partir d'Aproz, dans la vallée du Rhòne, jusqu'à Veisonnaz, Cleibe, Bleusy, les Mayens de Nendaz, la Crête et au-dessus de Haute-Nendaz. L'embouchure du Val de Nendaz étant beaucoup plus ouverte et mieux exposée que celle du Val Ferret, la région inférieure des vallées atteint normalement une altitude un peu plus grande $\left(1300^{\mathrm{m}}\right.$ environ, variant suivant les stations entre 1250 et $\left.1350^{\mathrm{m}}\right)$, et, à cause de l'exposition plus uniforme, le phénomène de l'envers et de l'endroit joue un ròle beaucoup moins considérable que dans cette dernière vallée; on peut constater tout au plus une différence de $50^{\mathrm{m}}$ entre l'endroit (jusqu'à $1350^{\mathrm{m}}$ au-dessus de Haute-Nendaz) et l'envers $\left(1300^{\mathrm{m}}\right.$ près de Cleibe et de Veisonnaz).

Hyalina glabra. Agriolimax alpestris. Arion subfuscus. Eulota fruticum. Pyramidula rupestris.
Chilotrema lapicida.

Helix pomatia.

Xerophila ericetorum.

Xerophila candidula.

Chondrula quadridens. 
Pupa avenacea.

Pupilla triplicata.

Succinea oblonga.

Limnaea limosa subsp. peregra.

Hyalina cellaria.

Arion empiricorum.

Arion hortensis.

Vallonia pulchella var. helvetica.
Arianta arbustorum.

Fruticicola strigella.

Tachea nemoralis.

Xerophila obvia.

Buliminus detritus.

Cochlicopa lubrica.

Pupilla muscorum.

Succinea pfeifferi.

Limnaea truncatula.

Pisidium fossarinum.

\section{Région inférieure des Foréts.}

Cette région comprend les quelques rares bois ou forèts compris entre Aproz et Bleusy ou les Mayens de Nendaz, ainsi que le bas des forêts qui recouvrent les flancs de la Dent de Nendaz (bas des Praz-Condjuz, après Haute-Nendaz), c'est-à-dire jusqu'à une altitude d'environ $1350-1400^{\mathrm{m}}$.

Limax maximus et 2 var.

Agriolimax agrestis.

Vitrina pellucida.

Hyalina cellaria.

Hyalina pura.

Euconulus fulvus.

Arion subfuscus.

Punctum pygmaeum.

Helicodonta obvoluta.

Fruticicola strigella.

Chilotrema lapicida.

Buliminus montanus.

Cochlicopa lubrica.

Clausilia dubia.

Clausilia ventricosa.
Limax tenellus.

Vitrina diaphana ${ }^{1}$.

Hyalina glabra.

Hyalina nitens.

Hyalina radiatula.

Arion empiricorum.

Arion hortensis.

Patula rotundata.

Fruticicola sericea.

Arianta arbustorum.

Helix pomatia.

Buliminus obscurus.

Vertigo pygmaea.

Balea perversa.

Clausilia plicatula.

${ }^{1} \mathrm{~J}$ 'hésite à considérer cette espèce comme habitant la région inférieure des forêts; elle ne se trouve jamais qu'à partir d'une certaine altitude, $1150-1200 \mathrm{~m}$ environ. Il est vrai que dans le Jura on la trouve a $700 \mathrm{~m}$. 


\section{RÉgion SUPÉrieure des Foréts.}

De la forêt des Praz-Condjuz, des Mayens de Nendaz, de Bleusy et de Cleibe, jusqu'à la limite supérieure des forêts, c'est-à-dire de 1300-1400 à 2000-2200 ${ }^{\mathrm{m}}$ environ. A la partie inférieure, les deux régions des forêts empiètent l'une sur l'autre sur environ $100^{\mathrm{m}}$ d'altitude, particulièrement aux Praz-Condjuz où les Helicodonta holoserica et obvoluta vivent en compagnie immédiate, souvent même sous une même pierre. Le long d'un sentier, j'ai trouvé l' $H$. holoserica au bas d'une pente, au sommet de laquelle vit l'H. obvoluta.

\section{Limax maximus.}

Limax tenellus.

Vitrina diaphana.

Hyalina nilens.

Hyalina pura.

Euconulus fulvus.

Arion hortensis.

Patula ruderata.

Fruticicola sericea.

Arianta arbustorum var. alpicola.

Buliminus obscurus.

Balea perversa.
L. maximus var. cinereo niger. Vitrina pellucida ${ }^{1}$.

Vitrina elongata var. sapinea.

H. nitens var. dutaillyana.

Hyalina radiatula.

Arion subfuscus.

Punctum pygmaeum.

$P$. ruderata var. viridana.

Helicodonta holoserica.

Buliminus montanus.

Vertigo alpestris.

Clausilia dubia.

\section{Région supérieure des vallées.}

Cette région occupe un espace relativement petit, limité de tous còtés par des forèts. C'est le fond de la vallée, après la forèt qui suit Bleusy, environ entre 1350 et $2000^{\mathrm{m}}$.

Limax maximus var. cinerea. Agriolimax agrestis.

Euconulus fulous.

Hyalina radiatula.

Arion subfuscus.

Limnaea truncatula.

Limnaea limosa subsp. peregra Pisidium fossarinum.

var. excerpta.

1 Jusqu'à $1400^{m}$. Elle doit se trouver plus haut, dans les forêts.

Rev. Suisse de Zool. T. 21. 1913. 


\section{RÉgion alpine.}

Parfois directement au-dessus de la région précédente (audessus de Tortin et dans le Val de Cleuson); d'autres fois audessus de la limite supérieure des forèts (sommet de la Dent de Nendaz, depuis la combe au-dessus dı Lac Noir). En général entre 2000 et $2700^{\mathrm{m}}$, jusqu'à $2735^{\mathrm{m}}$ entre le Mont-Gond et le Mont-Gelé, voire mème jusqu'à $2900^{\mathrm{m}}$ près du Grand-Désert.

Vitrina pellucida var. alpina. Punctum pygmaeum.

Arion subfuscus.

Pyramidula rupestris.

Tachea sylvatica var. montana. Pupilla alpicola var. saxetana. Vertigo alpestris.

Limnaea truncatula.

Arianta arbustorum var. alpicola.

Pupilla alpicola var. saxetana.
Clausilia dubia var. alpiccla. Pisidium fossarinum.

Euconulus fulvus.

\section{Vi. Région subnivale.}

La Vitrina nivalis et l'Arion subfuscus vivent en compagnie l'une de l'autre près des taches de neige, dans une combe au flanc de la Dent de Nendaz, au-dessus du Lac Noir $\left(2350-2460^{\mathrm{m}}\right.$ environ). Ces deux espèces suivent sans doute toute la crète jusqu'au Mont-Gelé, car on les retrouve dans des conditions semblables des deux còtés d'un col surplombant le lac des Vaux, au-dessus de Tortin, près du Mont-Gond. Au GrandDésert, la Vitrina pellucida var. alpina se trouve soit dans la région alpine, soit dans la région subnivale, entre 2800 et $2900^{\mathrm{m}}$.

\section{d) Le Val d'Hérens.}

\section{Région inférieure des vallées.}

Cette région comprend la fin de la vallée, soit dans le fond depuis Bramois jusqu'à près d'Evolène, soit sur les deux ver- 
sants jusqu'à Nax, Vernamiège et Mage d'une part, et Hérémence et les Mayens de Sion, d'autre part (au-desslis d'Euseigne et de Vex), c'est-à-dire jusqu'à une altitude d'environ 1300-1350 ${ }^{\mathrm{m}}$. De Mage et d'Hérémence, la limite supérieure de cette région, au lieu de rester à environ $1300^{\mathrm{m}}$, descend assez brusquement au fond de la vallée, à Luette, où l'on trokve l'Arianta arbustorum normale, alors que juste au-dessus, sur les versants, à SaintMartin par exemple, on ne trouve plus que la var. alpicola à $1250-1300^{\mathrm{m}}$. Ce fait s'explique par la mauvaise exposition résultant du resserrement de la vallée depuis Euseigne et Luette, qui ne permet plus à cette région inférieure que de s'étendre au fond de la vallée en abandonnant les flancs.

Arion empiricorum.

Arion horlensis.

Vallonia pulchella var. helvetica.

Chilotrema lapicida.

Tachea nemoralis.

Xerophila obvia.

Xerophila candidula.

Chondrula quadridens.

Pupa avenacea.

Pupilla muscorum.

Isthmia muscorum.

Succinea oblonga.

Limnaea limosa subsp. peregra et var.
Agriolimax agrestis.

Hyalina glabra.

Arion subfuscus.

Pyramidula rupestris.

Arianta arbustorum.

Helix pomatia.

Tachea sylvatica.

Xerophila ericetorum.

Buliminus detritus.

Cochlicopa lubrica.

Pupa sariabilis $\left(1000^{\mathrm{m}}\right)$.

Pupilla triplicata.

Succinea pfeifferi.

Limnaea truncatula.

Pisidium fossarinum.

II. Région inférieure des forèts.

Cette région paraît subir l'influence du resserrement de la vallée, depuis Luette, encore plus que la région précédente. En effet, tandis que sur les flancs, aux Mayens de Sion, à Nax, Vernamiège et Mage, la région inférieure des forèts atteint 
$1250-1300^{\mathrm{m}}$, au fond de la vallée elle ne dépasse pas $1000^{\mathrm{m}}$ près de Luette, où j'ai trouvé l'Arianta arbustorum normale en compagnie de l'Helicodonta holoserica. Certain€s espèces montent mème jusqu'à $1400^{\mathrm{m}}$ sur le versant des Mayens de Sion (endroit).

Limax maximus et 2 var.

Agriolimax ugrestis.

Vitrina pellucida.

Hyalina glabra.

Hyalina pura.

Euconulus fulvus.

Arion subfuscus.

Punctum pygmaeum.

Patula rotundata.

Fruticicola sericea.

Fruticicola strigella.

Chilotrema lapicida.

Tachea sylvatica.

Buliminus obscurus.

Clausilia dubia.

Clausilia plicatula.
Limax tenellus.

Vitrina diaphana.

Vitrina annularis.

Hyalina nitens.

Hyalina radiatula.

Arion empiricorum.

Arion hortensis.

Pyramidula rupestris.

Helicodonta obvoluta.

Fruticicola hispida.

Arianta arbustorum.

Buliminus montanus.

Cochlicopa lubrica.

Balea perversa.

Clausilia ventricosa.

Le Pomatias septemspirale doit sans doute aussi se trouver dans cette région.

\section{Région Supérieure des foréts.}

Toutes les forêts depuis les Mayens de Sion, Hérémence, la Luette, Saint-Martin, Mage, Vernamiège et Nax, jusqu'à leur limite supérieure. Comme dans toutes les vallées, il y a une zone où les deux régions des forêts se confondent $\left(1300-1400^{\mathrm{m}}\right)$.

Limax maximus.

Limax tenellus.

Vitrina elongata var. sapinea. H. nilens var. dutaillyana.
Arion subfuscus.

Punctum pygmaeum.

Patula ruderata et var.

Fruticicola sericea. 
Arianta arbustorum var. alpi- Arion hortensis. cola.

Buliminus montanus.

Pyramidula rupestris.

Cochlicopa lubrica. Helicodonta holoserica.

Fruticicola strigella.

Vertigo arctica.

Helix pomatia et var.

Clausilia dubia.

Buliminus obscurus.

L. maximus var. cinereo niger. Verligo alpestris.

Vilrina diaphana.

Balea perversa.

Hyalina nitens.

Clausilia plicatula.

Euconulus fulvus.

Pomatias septemspirale.

\section{Région supérieure des vallées.}

Cette région comprend le fond de la vallée avec une petite partie des versants, depuis Evolène jusqu'aux forèts de Ferpècle ou d'Arolla.

Agriolimax agrestis.

Arion subfuscus.

Arianta arbustorum var. alpicola.

Xerophila ericelorum $\left(1700^{\mathrm{m}}\right)$. Chondrula quadridens $\left(1870^{\mathrm{m}}\right)$.
Arion empiricorum ${ }^{1}$.

Arion hortensis.

Helix pomatia.

Buliminus detritus $\left(1750^{\mathrm{m}}\right)$.

Limnaea limosa subsp. peregra var. ullepilschi.

\section{Région alpine.}

Cette région comprend les pàturages situés au-dessus de la limite des forèts (Alpe de Veisivi et Crète de Thyon).

Arianta arbustorum var. alpi- Pisidium fossarinum. cola.

${ }^{1}$ Cette espèce occupe-t-elle à proprement parler la région supérieure des vallées? Elle n'atteint que $1450^{\mathrm{m}}$ aux Haudères. 


\section{Région subnivale.}

Je n'ai que très peu parcouru ces deux dernières régions. Aux abords immédiats du glacier d'Arolla ou de Bertol, je n'ai trouvé que

Limax tenellus. Arion subfuscus.

Patula ruderata.

e) Environs de Loèche-les-Bains.

\section{RÉgion inférieure des VAllées.}

La région inférieure des vallées proprement dite ne monte que jusqu'à $1200-1300^{\mathrm{m}}$ environ, et ne serait caractérisée que pár l'Arianta arbustorum normale et par la Tachea nemoralis. En effet, la plupart des espèces qui, dans le Val Ferret et dans le Val de Nendaz, sont spéciales à la région inférieure, dépassent de beaucoup cette limite aux environs de Loèche-les-Bains, à cause de la situation très favorable de cette vallée, au nord du Rhòne. Mais il est étonnant de comparer la limite supérieure de ces espèces au Val d'Hérens, situé au sud du Rhòne, par conséquent moins bien exposé, et à Loèche-les-Bains :

$\begin{array}{lll}\text { Arion empiricorum } & 1450^{\mathrm{m}} & 1450^{\mathrm{m}} \\ \text { Fruticicola strigella } & 1700 & 1600 \\ \text { Xerophila ericetorum } & 1700 & 1600 \\ \text { Buliminus detritus } & 1750 & 1600 \\ \text { Chondrula quadridens } & 1870 & 1600\end{array}$

Ces faits, en apparence contradictoires, sont pourtant le résultat d'obligations matérielles résultant de la longueur des vallées. Il est évident que, la vallée de Loèche étant plus de 
deux fois plus courte que le Val d'Hérens avec ses ramifications d'Arolla et de Ferpècle, les cinq espèces susnommées ne pourraient qu'avec peine dépasser les altitudes auxquelles elles arrivent. Considéré ainsi, le rapport de ces cinq limites supérieures dans les deux vallées n'a plus rien que de très naturel et confirme mème tout à fait la loi des embranchements latéraux du Valais, d'exposition différente suivant leur situation méridionale ou septentrionale. Qu'on examine, en effet, les distributions hypsométriques de ces cinq espèces dans le Val de Nendaz, déjà passablement plus long que la vallée de Loèche, ou surtout dans le Val Ferret. (Voir plus loin, Tableau, etc.) Du reste, le fait de la répartition altitudinaire de la Tachea nemoralis est bien caractéristique à ce sujet.

Voici les espèces de la région inférieure des vallées :

Agriolimax agrestis.

Hyalina cellaria.

Arion horlensis.

Fruticicola strigella.

Helix pomatia.

Tachea sylvatica.

Xerophila candidula.

Chondrula quadridens.

Clausilia ventricosa.
Hyalina glabra.

Arion empiricorum.

Pyramidula rupestris.

Arianta arbustorum.

Tachea nemoralis.

Xerophila ericetorum.

Buliminus detritus.

Cochlicopa lubrica .

Pupa avenacea.

La Limnaea limosa subsp. peregra a été trouvée à $1400^{\mathrm{m}}$ environ.

\section{Région inférieure des foréts.}

Cette région est mieux délimitée que la précédente, car, alors que certaines des espèces précédentes ne peuvent pas dépasser telle limite à cause de la nature du terrain, les forêts par contre s'étendent jusqu'à $1850-1900^{\mathrm{m}}$, et il est facile de constater la limite des espèces inférieures. Les différences entre la distribution hypsométrique des espèces vivant au nord et au sud 
du Rhòne sont beaucoup plus accentuées. Voir, plus haut, les généralités.) La région inférieure des forèts s'étend donc jusqu'à une altitude de $1400-1600^{\mathrm{m}}$, aux environs de Loèche-lesBains, dans les forèts des bords de la Dala, d'Allmend, etc.

Limax maximus.

Agriolimax agrestis.

Vitrina diaphana.

Hyalina cellaria.

Hyalina pura.

Arion empiricorum.

Arion hortensis.

Palula rotundala $\left(1600^{\mathrm{m}}\right)$.

Helicodonta obvoluta.

Fruticicola strigella.

Helix pomatia.

Buliminus obscurus.

Cochlicopa lubrica.

Balea perversa.

Clausilia dubia et var.

Clausilia plicatula.
Limax tenellus.

Vitrina pellucida.

Hyalina glabra.

Hyalina nilens.

Euconulus fulous.

Arion subfuscus.

Sphyradium edentulum.

Pyramidula rupestris.

Fruticicola sericea.

Arianta arbustorum.

Buliminus montanus.

Acanthinula aculeata.

Pupa secale.

Clausilia laminata.

Clausilia parvula.

ili. Région Supérieure des foréts.

Cette région comprend toutes les forèts à partir de $1400-1600^{\mathrm{m}}$ jusqu'à leur limite supérieure.

Limax maximus.

Agriolimax agrestis.

Vitrina diaphana.

Hyalina cellaria ${ }^{1}$.

Hyalina pura.

Arion subfuscus.

Patula ruderata.
Helicodonta holoserica.

Arianta arbustorum var. alpicola.

Buliminus montanus.

Pupa secale.

Clausilia laminata.

Clausilia cruciala et var. 
Limax tenellus.

Vitrina pellucida.

Hyalina glabra ${ }^{1}$.

Hyalina nitens.

Euconulus fulvus.

Arion hortensis.

Pyramidula rupestris.
Fruticicola sericea.

Tachea sylvatica var. montana. Buliminus obscurus.

Balea perversa.

Clausilia dubia et var.

Clausilia plicatula ${ }^{1}$.

\section{Région súpérieure des vallées.}

Cette région comprend la partie supérieure de la vallée, au pied des parois de rochers de la Gemmi, à l'Alpe de Clavinen et aux environs de Planetry, environ 1750-1800 à 1900-1950 .

Agriolimax agrestis.

Arion subfuscus.

Pyramidula rupestris.

Helix pomatia.

Cochlicopa lubrica.
Euconulus fulvus.

Arion hortensis.

Arianta arbustorum var. alpicola.

Tachea sylvatica var. montana.

\section{Région alpine.}

Cette région comprend les parois rocheuses de la Gemmi, à partir d'une altitude d'environ $2000^{\mathrm{m}}$ jusqu'à peu près $2300^{\mathrm{m}}$. Voici les espèces qui s’y trouvent :

Limax tenellus. Jusqu'à $2100^{\mathrm{m}}$.

Agriolimax agrestis. On la trouve en compagnie de l'espèce précédente, jusqu'à la mème altitude.

Vitrina diaphana. Jusqu'à 2300"m (Bollinger).

Zoniloides nitidus. 2000' (STUDER).

Arion subfuscus. Jusqu'à $2300^{\mathrm{m}}$.

Sphyradium gredleri. 2300 ${ }^{\mathrm{m}}$ (Chessin).

Arianta arbustorum var. alpicola. Jusqu'à $2330^{\mathrm{m}}$.

Tachea sylvatica var. montana. Jusqu'à $2330^{\mathrm{m}}$.

$1700^{\mathrm{m}}$. 


\section{Région subNivale.}

Cette région occupe certaines parties de la crête de la Gemmi entre 2300 et $2350^{\mathrm{m}}$.

Arion subfuscus.

Voici enfin un tableau destiné à faciliter les comparaisons entre les différentes limites hypsométriques des genres, espèces et variétés, suivant les cinq vallées étudiées. Il indique en outre la distribution verticale dans les six régions altitudinaires, d'une manière générale. 


\begin{tabular}{|c|c|c|}
\hline \multirow{2}{*}{ 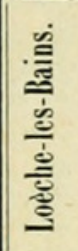 } & $\begin{array}{c}\text { ·dns } \\
\text { ә|!ฺ!' }\end{array}$ & 产导 | | \\
\hline & 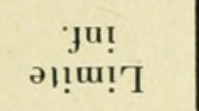 & $1|1| 1|1| 1|1 \stackrel{8}{\circ}| 1|1| 1|1| 1|1| 1 \mid 1$ \\
\hline \multirow{2}{*}{ 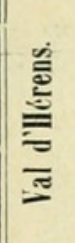 } & $\begin{array}{c}\text { dns } \\
\text { ә!ฺщu!T }\end{array}$ & 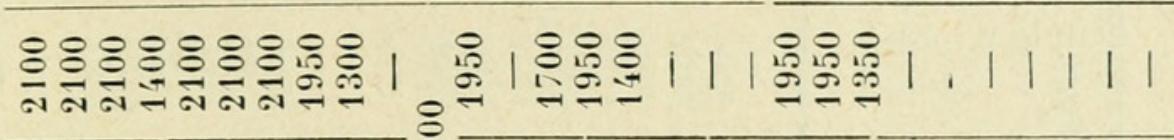 \\
\hline & $\begin{array}{c}\text { ju! } \\
\text { ә!ฺแฺ! }\end{array}$ & 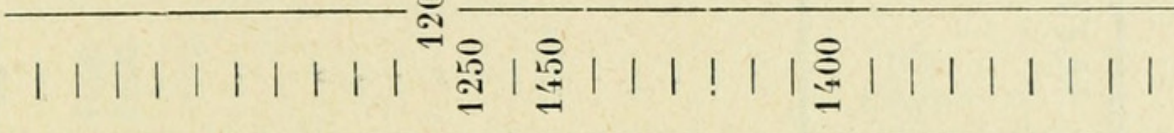 \\
\hline \multirow{2}{*}{ 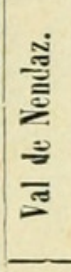 } & $\begin{array}{c}\text { dns } \\
\text { ә!!ш!T }\end{array}$ & | \\
\hline & $\begin{array}{c}\text { ju! } \\
\text { ә!!ய! T }\end{array}$ & 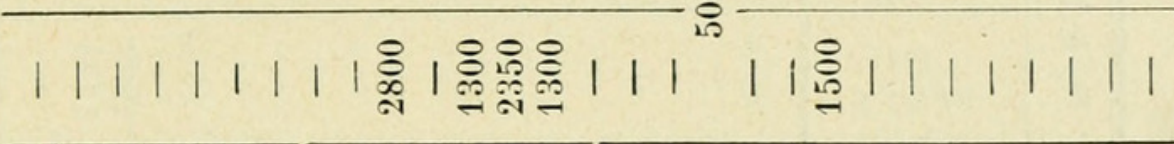 \\
\hline \multirow{2}{*}{ 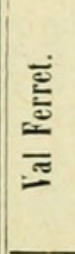 } & $\begin{array}{c}\cdot \text { dns } \\
\text { ว!!ณ!'T }\end{array}$ & 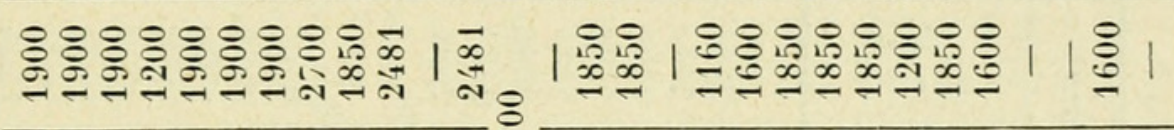 \\
\hline & 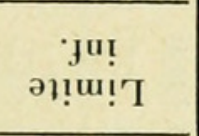 & 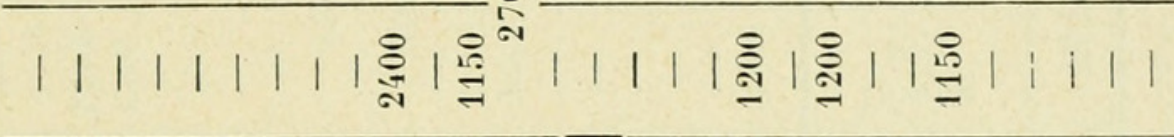 \\
\hline \multirow{2}{*}{ 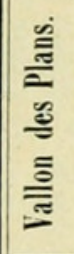 } & $\begin{array}{l}\text { dns } \\
\text { ว!!u!'T }\end{array}$ & 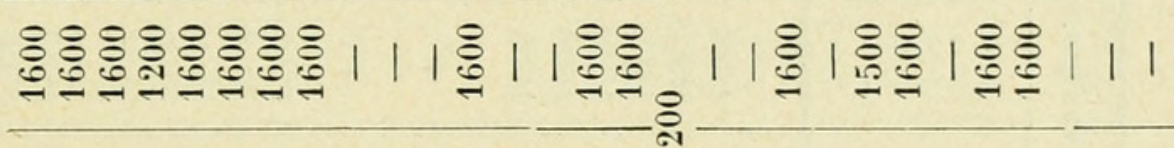 \\
\hline & 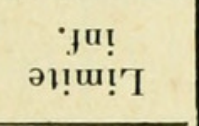 & $|1| 1|1| 1|1| \frac{8}{8}|1| 1 /$, $|1| 1|1| 1|1| 1 \mid$ \\
\hline \multicolumn{2}{|c|}{$\cdot$ ว[es!uqn $\cdot 1 \Lambda$} & ++++++ \\
\hline & $\cdot$ วu!d $_{I V} \cdot \Lambda$ & +++++ \\
\hline \multicolumn{2}{|c|}{ 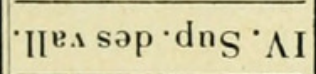 } & $+\quad+++$ \\
\hline \multicolumn{2}{|c|}{$\cdot . \log \mathrm{s} \partial \mathrm{p} \cdot \mathrm{dn} \mathrm{S} \cdot \mathrm{III}$} & +++++++++++++ \\
\hline \multicolumn{2}{|c|}{$\overline{\therefore 0 y \text { sәp } \cdot \mathrm{ju}^{\prime} \cdot \mathrm{II}}$} & +++++++++ \\
\hline \multicolumn{2}{|c|}{ 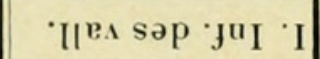 } & +++ \\
\hline & 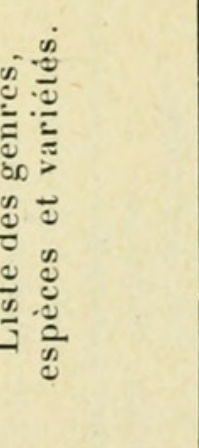 & 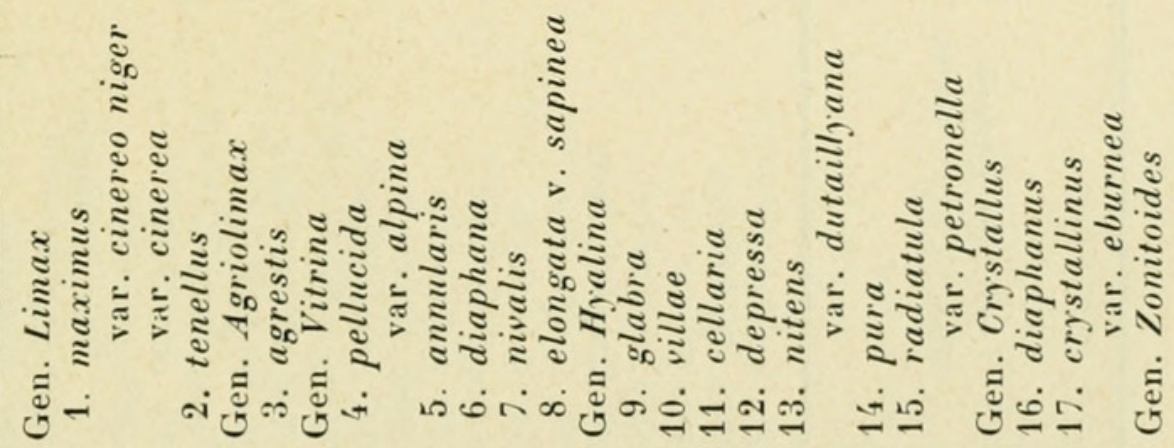 \\
\hline
\end{tabular}




\begin{tabular}{|c|c|c|}
\hline 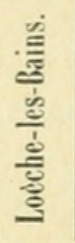 & $\begin{array}{l}\text { dns } \\
\text { ә!!u!T }\end{array}$ & 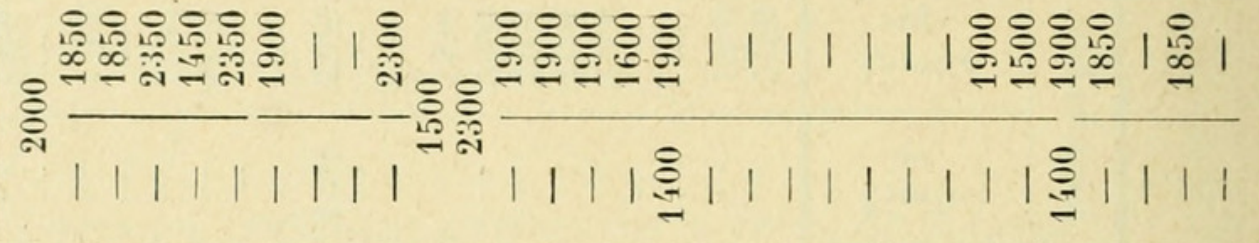 \\
\hline \multirow{2}{*}{ 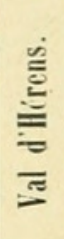 } & $\begin{array}{l}\cdot \text { dns } \\
\text { ว!!u!T }\end{array}$ & | \\
\hline & 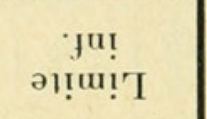 & 1111111111 \\
\hline \multirow{2}{*}{ 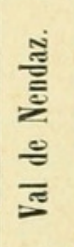 } & $\begin{array}{l}\text { dns } \\
\text { əิ!ฺ! }\end{array}$ & | \\
\hline & 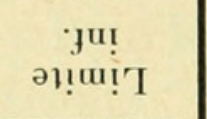 & $1111: 1111$ \\
\hline \multirow{2}{*}{ 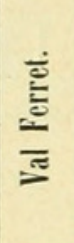 } & $\begin{array}{l}\cdot d n s \\
\text { ว!!u!'T }\end{array}$ & 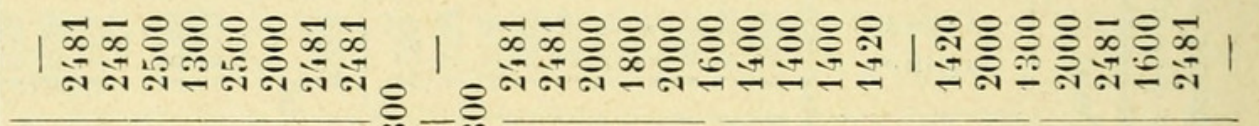 \\
\hline & 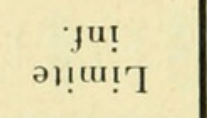 & $|1| 1|1| 1||^{-1} 11$ \\
\hline \multirow{2}{*}{ 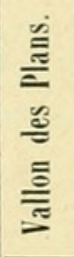 } & $\begin{array}{l}\text { dns } \\
\text { วเ!ณ!' }\end{array}$ & | \\
\hline & $\begin{array}{c}\text { јu! } \\
\text { әฺ!щ!т }\end{array}$ & ||$\overbrace{\overparen{D}}^{8}|1| 1$ \\
\hline \multicolumn{2}{|c|}{ 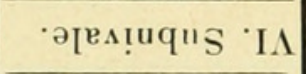 } & ++++ \\
\hline & $\cdot \operatorname{au}^{\prime} \mathrm{d}_{I \mathrm{~V}} \cdot \Lambda$ & +++++++++++ \\
\hline \multirow{2}{*}{\multicolumn{2}{|c|}{ 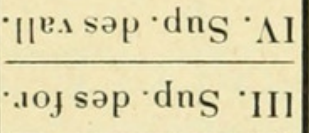 }} & +++++ \\
\hline & & ++++++++++++++ \\
\hline \multicolumn{2}{|c|}{$\cdot$ doj sәp ·رUI 'II } & $+++++++++\quad+++$ \\
\hline \multicolumn{2}{|c|}{ iles sap juा $\mathrm{I}$} & ++++ \\
\hline & 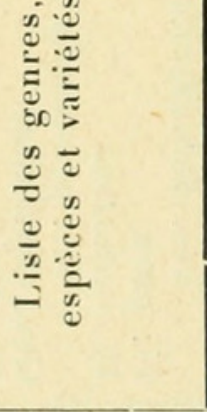 & 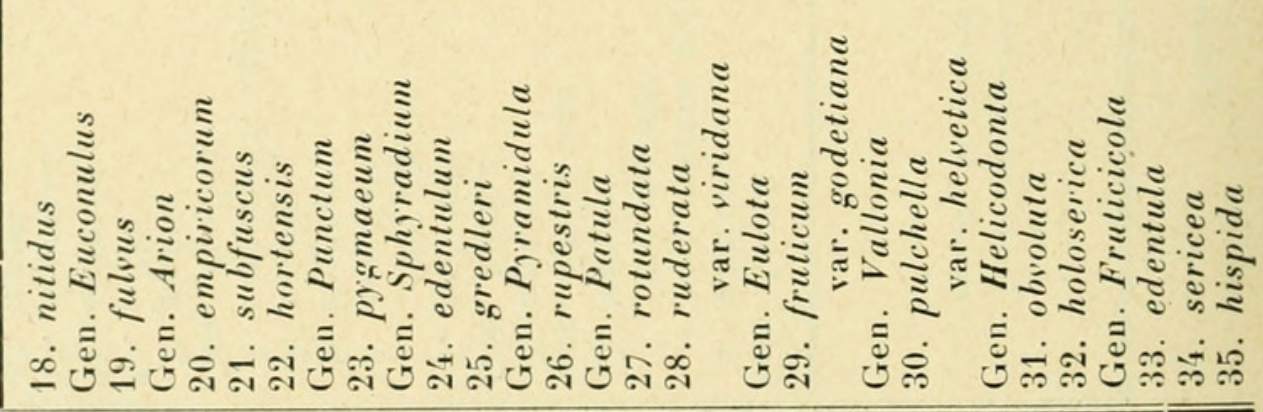 \\
\hline
\end{tabular}




\begin{tabular}{|c|c|c|}
\hline \multirow{2}{*}{ 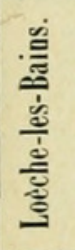 } & $\begin{array}{l}\cdot d n s \\
\text { ว!!u!'I }\end{array}$ & 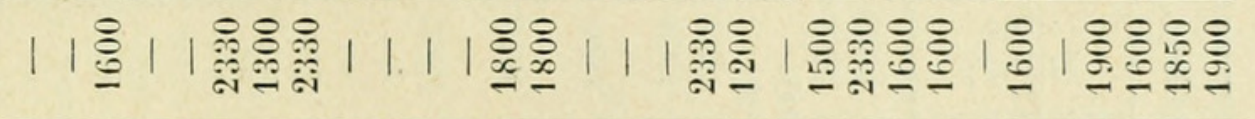 \\
\hline & 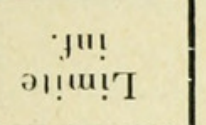 & | | | | | \\
\hline \multirow{2}{*}{ 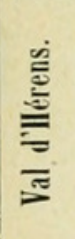 } & $\begin{array}{l}\text { dus } \\
\text { ә!!ฺ!t }\end{array}$ & | | \\
\hline & 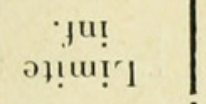 & 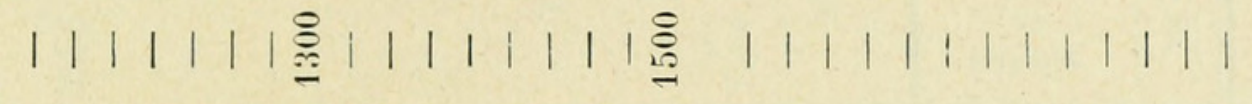 \\
\hline \multirow{2}{*}{ 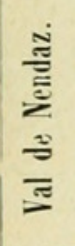 } & $\begin{array}{l}\text { dns } \\
\text { әा!เu!T }\end{array}$ & 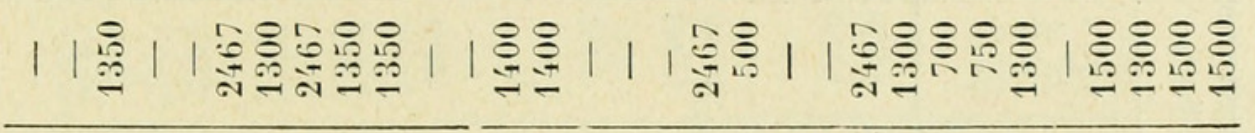 \\
\hline & 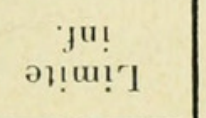 & 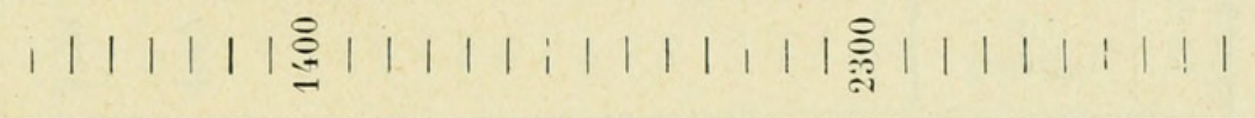 \\
\hline \multirow{2}{*}{ 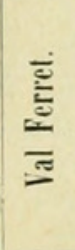 } & $\begin{array}{l}\text { dns } \\
\text { əІ!!u!'I }\end{array}$ & 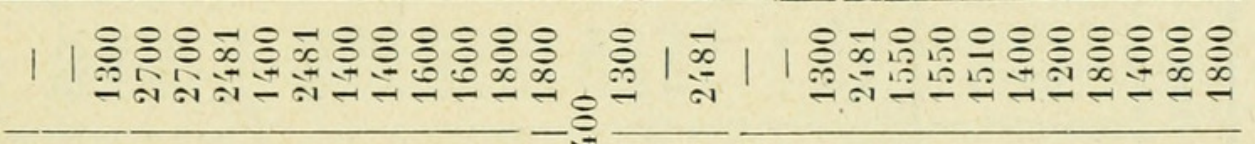 \\
\hline & 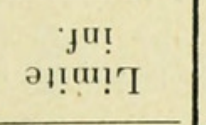 & 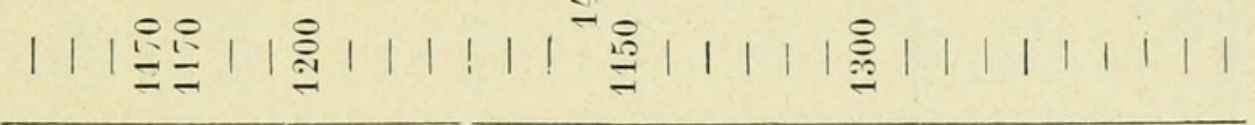 \\
\hline \multirow{2}{*}{ 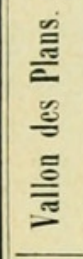 } & $\begin{array}{l}\cdot d n s \\
\text { วิเய!า }\end{array}$ & 号 \\
\hline & 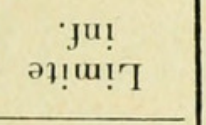 & 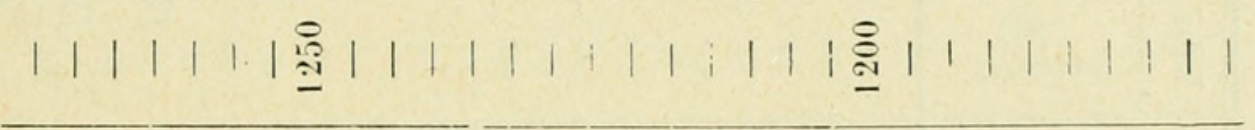 \\
\hline \multicolumn{2}{|c|}{ 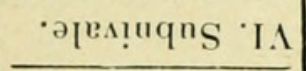 } & + \\
\hline & $\cdot \operatorname{antg}^{2} \cdot \Lambda$ & ++ \\
\hline \multirow{2}{*}{\multicolumn{2}{|c|}{ 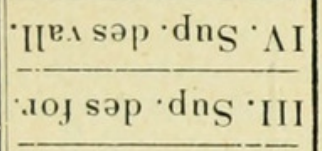 }} & ++++ \\
\hline & & ++++++++++ \\
\hline \multicolumn{2}{|c|}{.707 sop juI } & $+++++++\quad+\quad++$ \\
\hline \multicolumn{2}{|c|}{ 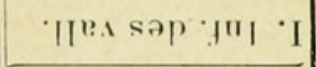 } & ++++++++++++++++ \\
\hline & 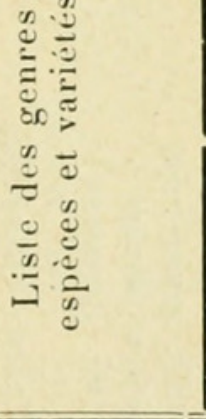 & 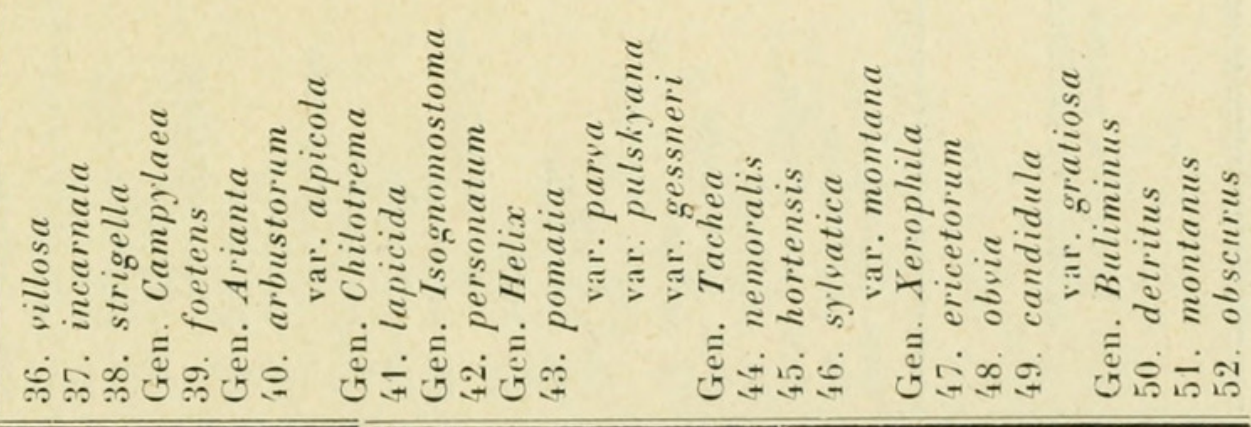 \\
\hline
\end{tabular}




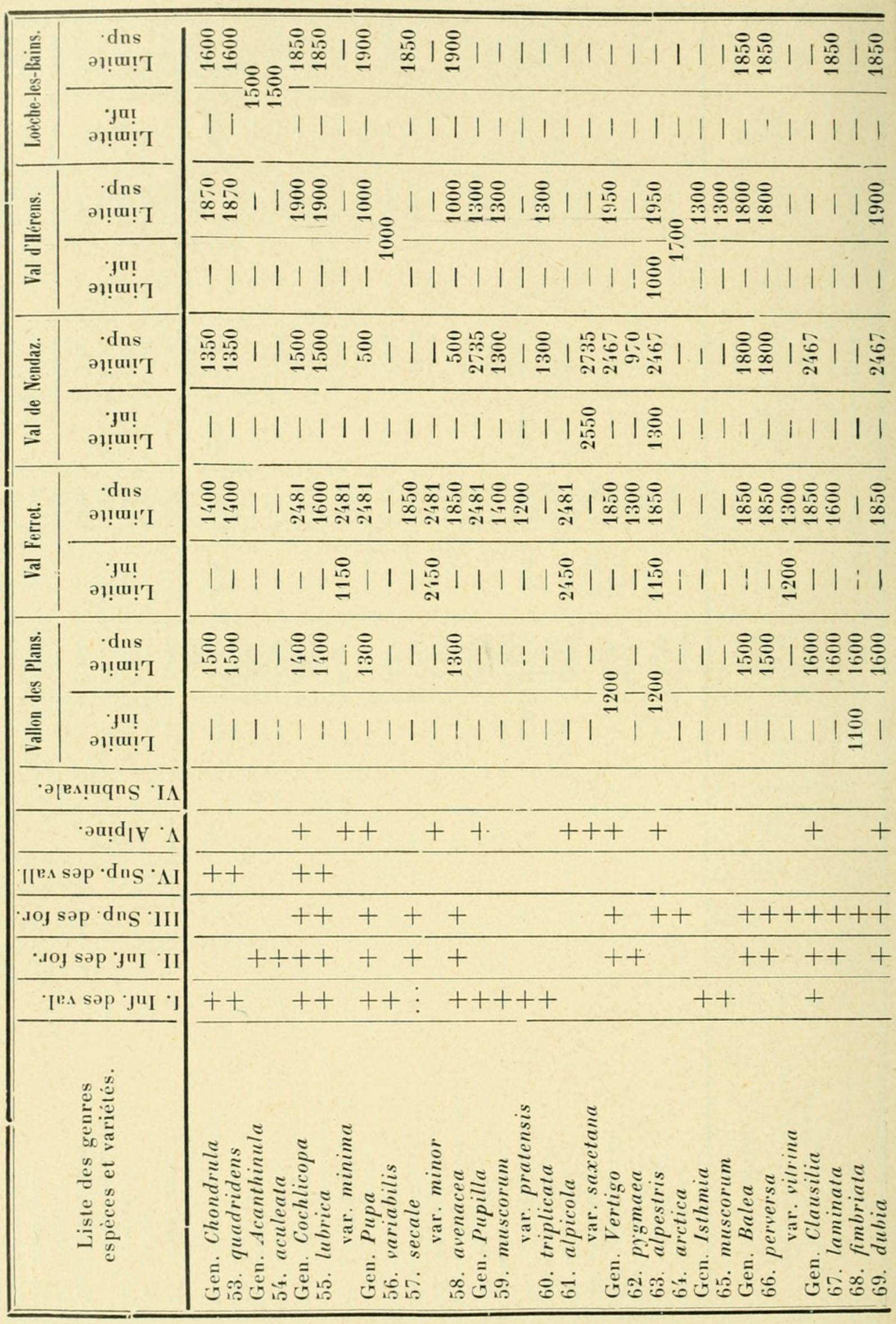




\begin{tabular}{|c|c|c|}
\hline \multirow{2}{*}{ 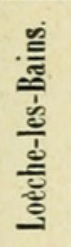 } & $\begin{array}{l}\text { ·dns } \\
\text { ว!!u!T }\end{array}$ & 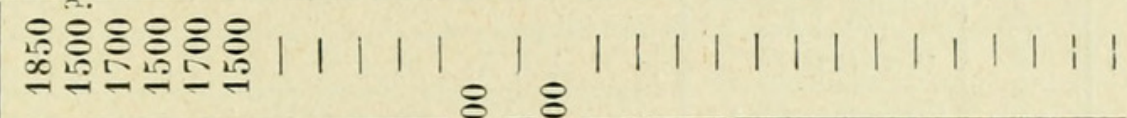 \\
\hline & 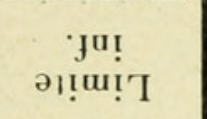 & $|1 \stackrel{8}{=}| 1|1| 1\left|1 / 1^{-}\right| 1|1| 1|1| 1|1| 1 \mid$ \\
\hline \multirow{2}{*}{ 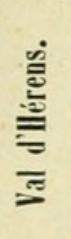 } & $\begin{array}{l}\text { ·dns } \\
\text { ә!ฺแ!T }\end{array}$ & 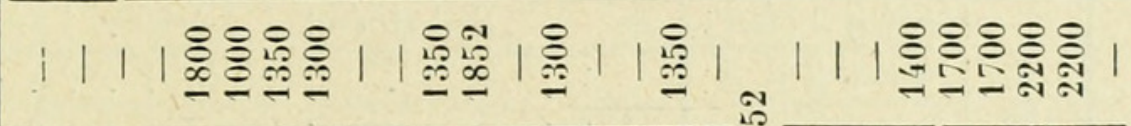 \\
\hline & 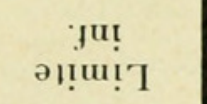 & $\left.|||||||||||||||| \stackrel{\mathscr{0}}{\circ}\right|^{-}|||| \mid$ \\
\hline \multirow{2}{*}{ 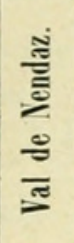 } & 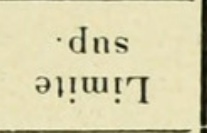 & 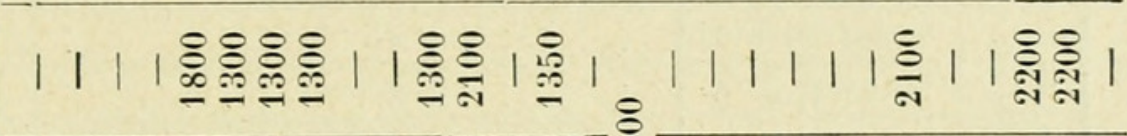 \\
\hline & 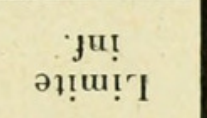 & $11|1| 1|1| 1|1| 1|1| 1|1| 1|1| 1 \mid 111$ \\
\hline \multirow{2}{*}{ 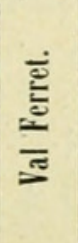 } & $\begin{array}{l}\text { dns } \\
\text { วย!u!T }\end{array}$ & $\mid$ | \\
\hline & 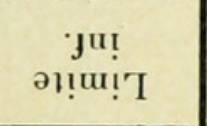 & 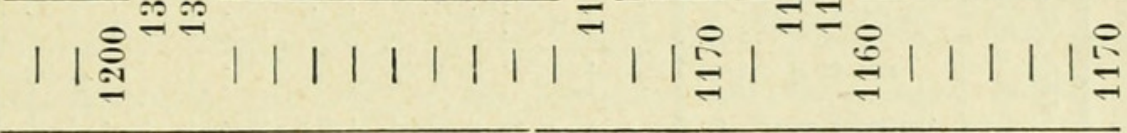 \\
\hline \multirow{2}{*}{ 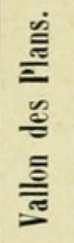 } & $\begin{array}{l}\cdot \mathrm{dns} \\
\text { วฺ!u!T }\end{array}$ & 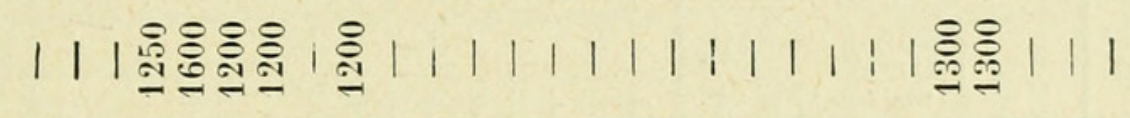 \\
\hline & 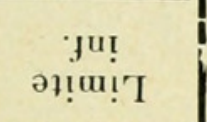 & 11111111111111111111 \\
\hline \multicolumn{3}{|c|}{ ·pex!uqne $I \Lambda$} \\
\hline \multicolumn{2}{|c|}{$\cdot \operatorname{sut} \mathrm{d}^{[\mathrm{t}} \cdot \mathrm{A}$} & +++ \\
\hline \multicolumn{2}{|c|}{$\cdot I l^{e} \Lambda$ sop $\cdot \operatorname{dn} S \cdot \Lambda I$} & $+\quad++$ \\
\hline \multicolumn{2}{|c|}{$\cdot d 0 j$ sap $\cdot d n s$ III } & ++++ \\
\hline \multirow{2}{*}{\multicolumn{2}{|c|}{ 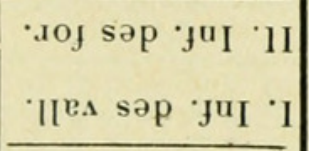 }} & $+\quad+++$ \\
\hline & & $+++++++++++++t$ \\
\hline & 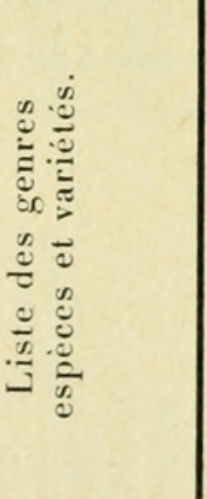 & 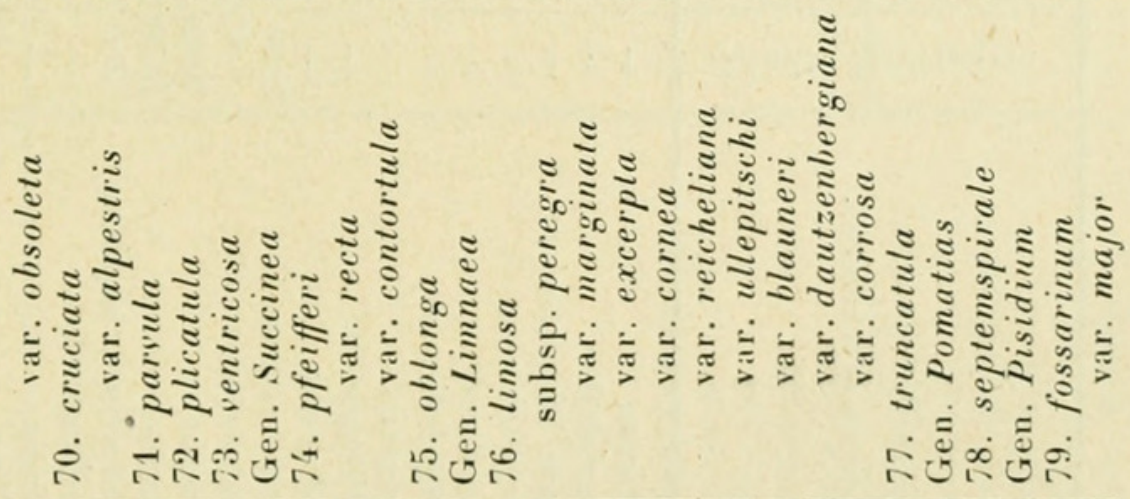 \\
\hline
\end{tabular}




\begin{tabular}{|c|c|c|}
\hline \multirow{5}{*}{ 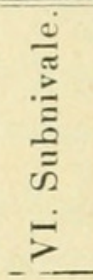 } & & $:::::::::: \cdot:::$ : \\
\hline & suә. & $+:::+::::::::::::^{\prime}::$ \\
\hline & zepu & 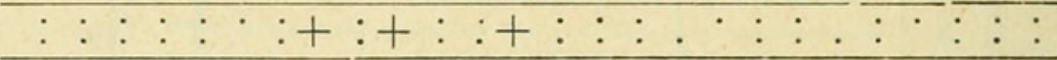 \\
\hline & ·....1.1 & $++: \cdot++++:::++:::::::: \vdots:: \vdots:$ \\
\hline & surid & 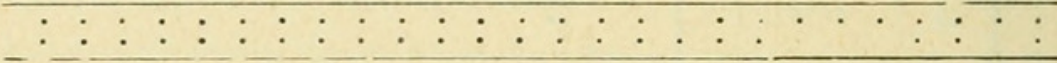 \\
\hline \multirow{5}{*}{$\begin{array}{l}\stackrel{\mathscr{g}}{\frac{\mathscr{g}}{2}} \\
\dot{\gamma}\end{array}$} & -suieg & $+:::++++:::+::: \cdot:: \cdot:::::$ \\
\hline & sua. & 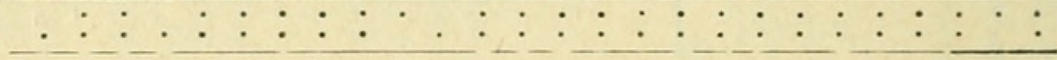 \\
\hline & zepu= & $:::::::+:+:::::::::::::^{0}$ \\
\hline & . $72.1 .1 \partial^{4} \Lambda$ & $:::::::+:+:+:::: \cdot:::::^{*}:{ }^{*}$ \\
\hline & suelc & 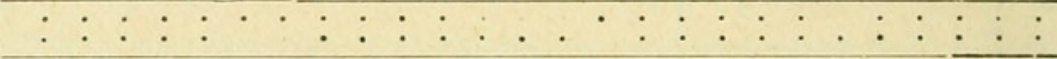 \\
\hline \multirow{5}{*}{ 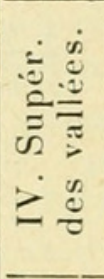 } & su! & $:::::++:::::::: \cdot:::::::$ \\
\hline & - sü & $::::++:::::::::::::::::::$ \\
\hline & 'zериә & $+::+:++: \cdot::::+:::::+: \vdots$ \\
\hline & ·. & 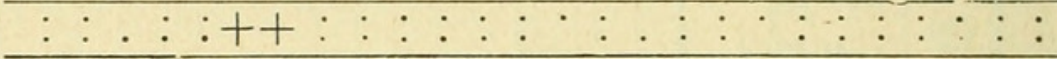 \\
\hline & $\cdot$ sue & 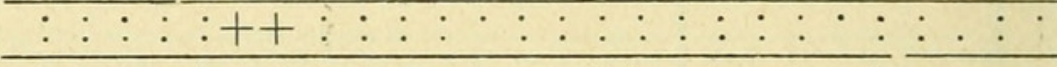 \\
\hline \multirow{5}{*}{ 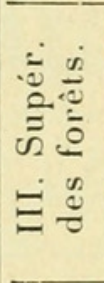 } & su & $++::+++++::+::++:+:+:+::::$ \\
\hline & suə & $+++:+\vdots:+: \vdots:+:+1:::++:: \vdots: \vdots$ \\
\hline & zepu & $+++:+::++::+:++::::++++:::$ \\
\hline & $\cdot 12.1 .1$ & $+++:+++++::+::++: \cdot++++:+t:$ \\
\hline & 'sue|d s & $+++:++++:::+::++:: \vdots+:++:++$ \\
\hline \multirow{5}{*}{ 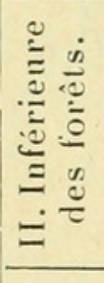 } & ·su! &.$++:+++++::+::++.+:+:+::::$ \\
\hline & 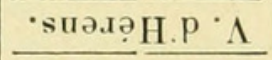 & $+++++++++:++: \cdot++:::+:++:::$ \\
\hline & ZериәN & $+++++++++::+::++:+:+.++::$ \\
\hline & - 7ә..1ว & $+++++++++::+::++:: \cdot+:++: t$ \\
\hline & suetd & $++++++++:::+::+++::+:++:++$ \\
\hline \multirow{6}{*}{ 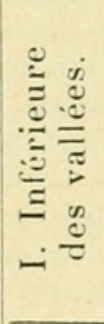 } & ' wo!s & 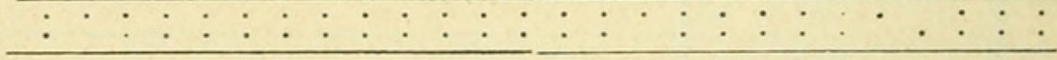 \\
\hline & - suḷeg & $:::::++:::::::++:+: \vdots:::^{0}$ \\
\hline & $\Lambda$ & 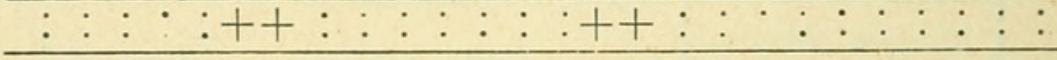 \\
\hline & zершә $\mathrm{N}$ әр $\Lambda$ & $:::::++: \cdot:::::++:+:: \vdots: \vdots: \vdots:$ \\
\hline & 7.1.1. & 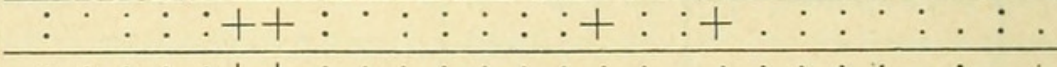 \\
\hline & 'sueId & $:: \quad: \quad: \quad: \quad: \quad: \quad: \quad: \quad$. \\
\hline \multicolumn{2}{|c|}{ 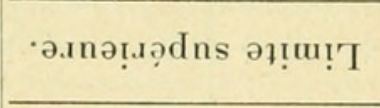 } & 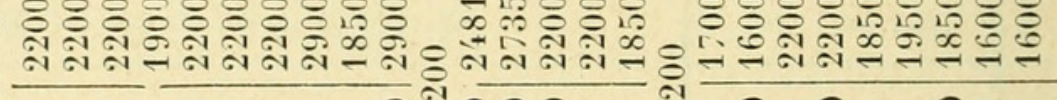 \\
\hline \multicolumn{2}{|c|}{ 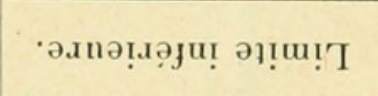 } & 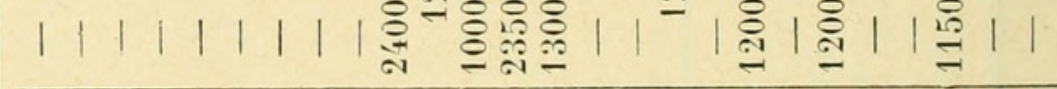 \\
\hline & 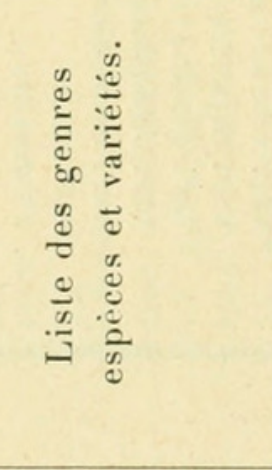 & 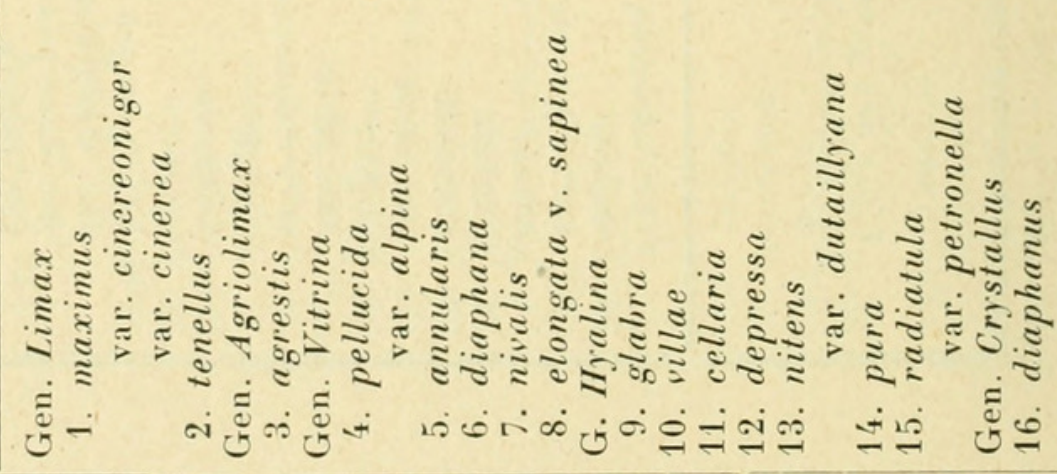 \\
\hline
\end{tabular}




\begin{tabular}{|c|c|c|}
\hline \multirow{5}{*}{ 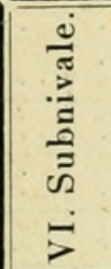 } & & + \\
\hline & & $::::+.+::::$ \\
\hline & 1 & $::::::+++:::::::::::$ \\
\hline & & $::::+++:+\cdot::::::+:+::::$ \\
\hline & sul & 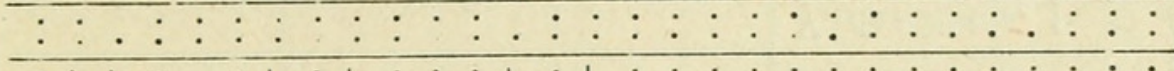 \\
\hline \multirow{5}{*}{ 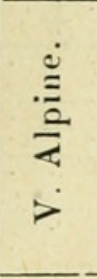 } & 'T & $:++: \cdot:+:+:: \vdots+:+:: \vdots:: \vdots: \vdots$ \\
\hline & $5 \pi$ & 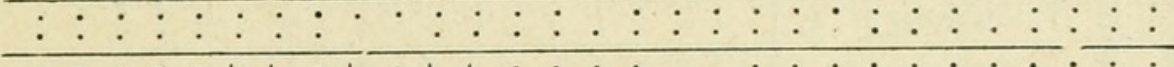 \\
\hline & & $:::::++:+:++:::: \cdot .:::::::::$ \\
\hline & & $\cdot::+++:++++:: t_{+}:::::::::::$ \\
\hline & sur & 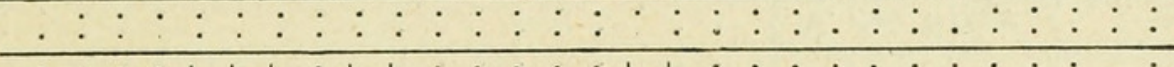 \\
\hline \multirow{5}{*}{ 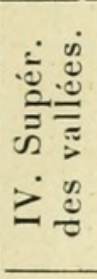 } & & 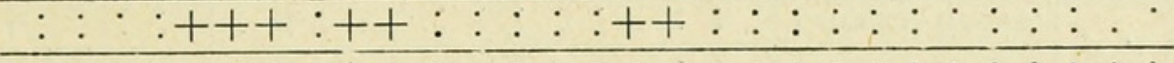 \\
\hline & $\cdot \mathrm{s} 1$ & $::::::++++1:::::::::::::::::::$ \\
\hline & $2 e_{1}$ & 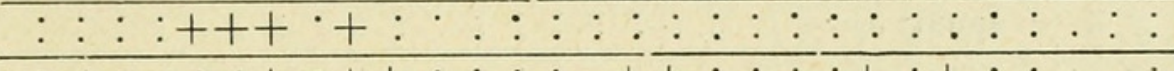 \\
\hline & & 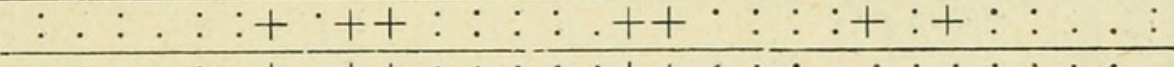 \\
\hline & 'sul & $::::::+:++::::: 1++::::::::::::$ \\
\hline \multirow{5}{*}{$\begin{array}{l}0 \\
\vdots \\
\vdots \\
0 \\
0\end{array}$} & & $::::+++++::::: t_{+}++t^{\circ}::::::::+$ \\
\hline & $\cdot \mathrm{si}$ & $:::: t_{+}+{ }^{+}++++:::+++:+::::::::+$ \\
\hline & zep & $::::+++{ }_{+}++++:::::+:++::::{ }^{\prime}::+$ \\
\hline & & $:+::+++:+++++:+++++++:::::$ \\
\hline & $\Lambda$ & $:: \cdot:+++:++:::::+++:+:::::::::+$ \\
\hline \multirow{5}{*}{ 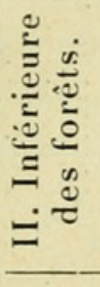 } & $\tau$ & $::::++++++::++:++++:::::::::{ }^{\prime}$ \\
\hline & & $::::++++++++:::++++:::::::::+$ \\
\hline & ${ }^{\cdot} \Lambda$ & $::::++++++++1::::++:::::::::+$ \\
\hline & & $:+::++++++++:::++++:::::::::+$ \\
\hline & - sue & $::::++++++:: .::++++::: \cdot:::::+$ \\
\hline \multirow{6}{*}{ 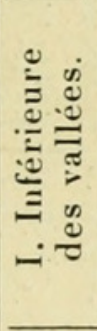 } & 可 & 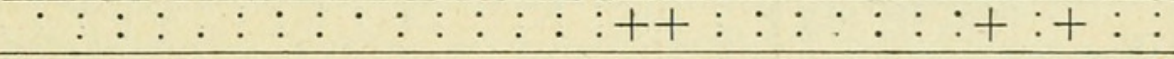 \\
\hline & sui & 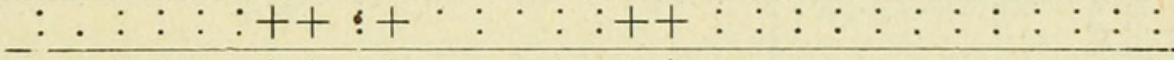 \\
\hline & & $:::::::++++:::::++:::::::+:$ \\
\hline & zepi & $\cdot:::::++++:::::++::::++:+:$ \\
\hline & '7.1. & $::::::++:+:::::++::::++:+::+$ \\
\hline & & $::::::++++:::::++::::++:::::$ \\
\hline \multicolumn{2}{|c|}{ 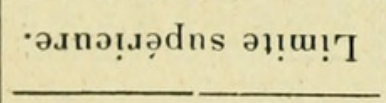 } & 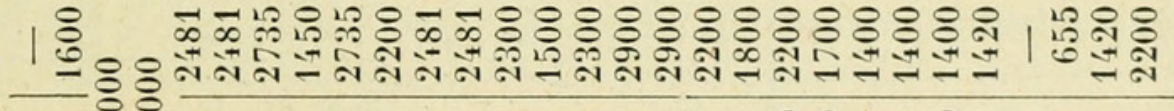 \\
\hline \multicolumn{2}{|c|}{ 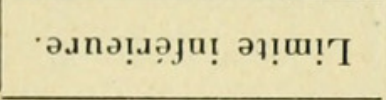 } & 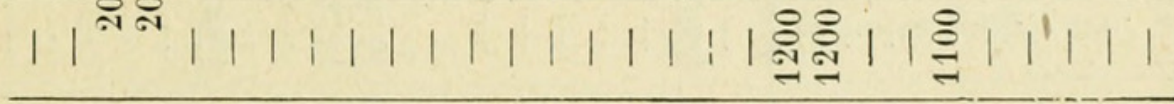 \\
\hline & 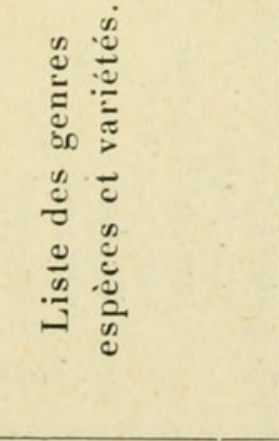 & 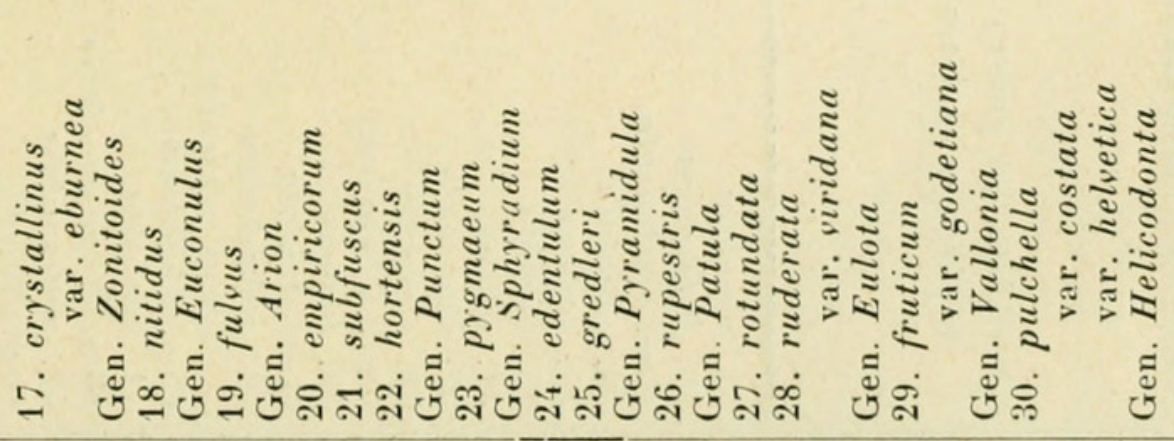 \\
\hline
\end{tabular}




\begin{tabular}{|c|c|c|}
\hline \multirow{5}{*}{ 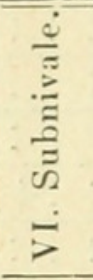 } & & $:::$ \\
\hline & suc & 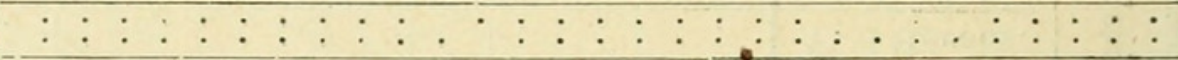 \\
\hline & & : : : : : : : : : : : : : : : : : : : : \\
\hline & & 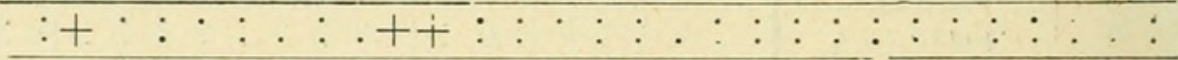 \\
\hline & & 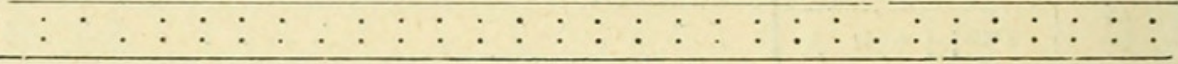 \\
\hline \multirow{5}{*}{$\begin{array}{l}\frac{.}{2} \\
\frac{2}{2} \\
>\end{array}$} & & $:::: \vdots:::::^{+}+:+: \vdots$ \\
\hline & & 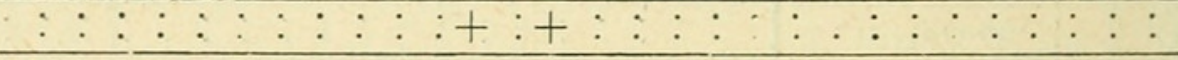 \\
\hline & $\Lambda$ & 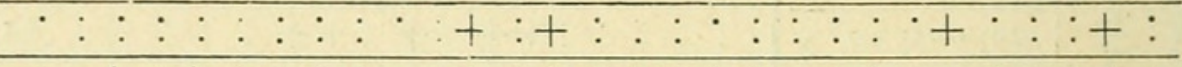 \\
\hline & & 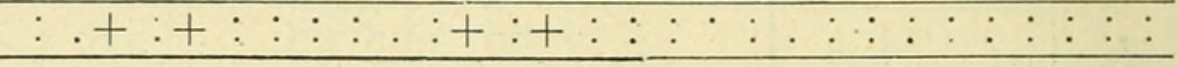 \\
\hline & & $: \vdots: \vdots: \vdots:: \vdots:: \vdots: \vdots:: \vdots$ \\
\hline \multirow{5}{*}{ 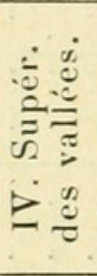 } & & $:::::::::::+:+:::_{+}:+:: t_{+}+$ \\
\hline & & $:::::::::::+.+: \cdot:++: \vdots::::::+$ \\
\hline & $\Lambda$ & 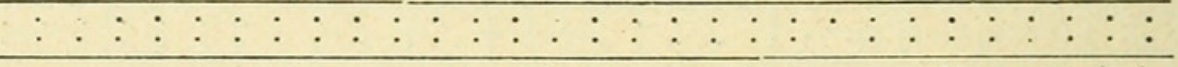 \\
\hline & & 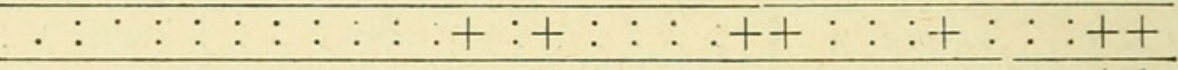 \\
\hline & & $:::::::::::+:+:: t_{+}+:::+:::++$ \\
\hline \multirow{5}{*}{ 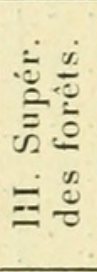 } & & $:++:+::::::+:+::::::: \cdot++:::+$ \\
\hline & & $+++:+::+1+1+::::++::+\ldots:::$ \\
\hline & & $:++:+: \cdot::::+:+:: \vdots$ \\
\hline & & $:++++:::: t++:+: t_{+}+:+::+:::+$ \\
\hline & & $:++:+::::::+1+::++:::::+:::+$ \\
\hline \multirow{5}{*}{ 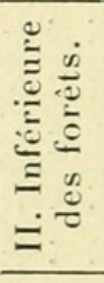 } & & $+:+:+:::+::++:::::++: \cdot \cdot::{ }^{\cdot}$ \\
\hline & & $+:+:++::+::++:++\cdot::::: .::$ \\
\hline & $\Lambda$ & $+:+:+:::+::++\cdot+::++::: \cdot::$ \\
\hline & & $+{ }^{\cdot}+++:::+::++:++++{ }^{\prime}::::: \quad: \quad:$ \\
\hline & & $+:+:+:++:::++:++++++:::+:++$ \\
\hline \multirow{6}{*}{ 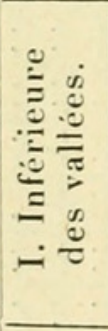 } & & $::+:: 0^{*}+:::::++::: \cdot::_{+}::+:+$ \\
\hline & & $::+:::: t^{2}::++:::::++:::++:+1+$ \\
\hline & $\Lambda$ & $:::: \vdots:: \vdots:::++:++: \vdots++:::+++1+$ \\
\hline & $\Lambda$ & $::+:::::+::++:++::++:::++:::+$ \\
\hline & & $::+:::::+::++:::: t_{+}+t_{+}:+:+\cdots+$ \\
\hline & $\cdot s$ & 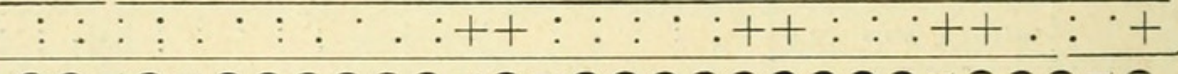 \\
\hline \multicolumn{2}{|c|}{ 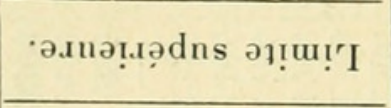 } & 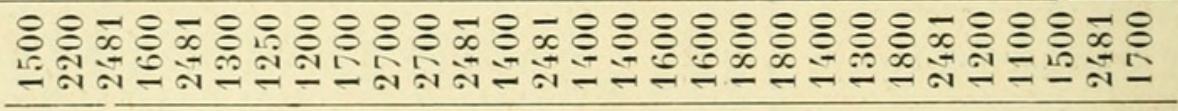 \\
\hline \multicolumn{2}{|c|}{ 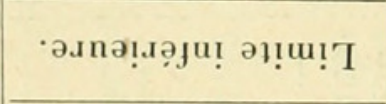 } & 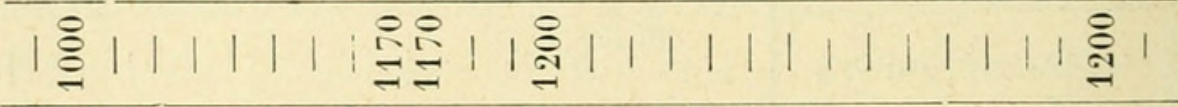 \\
\hline & $\begin{array}{cc}\infty & 0 \\
0 & 0 \\
0 & 0 \\
0 & 0 \\
\frac{0}{0} & 0 \\
\frac{0}{3} & 0\end{array}$ & 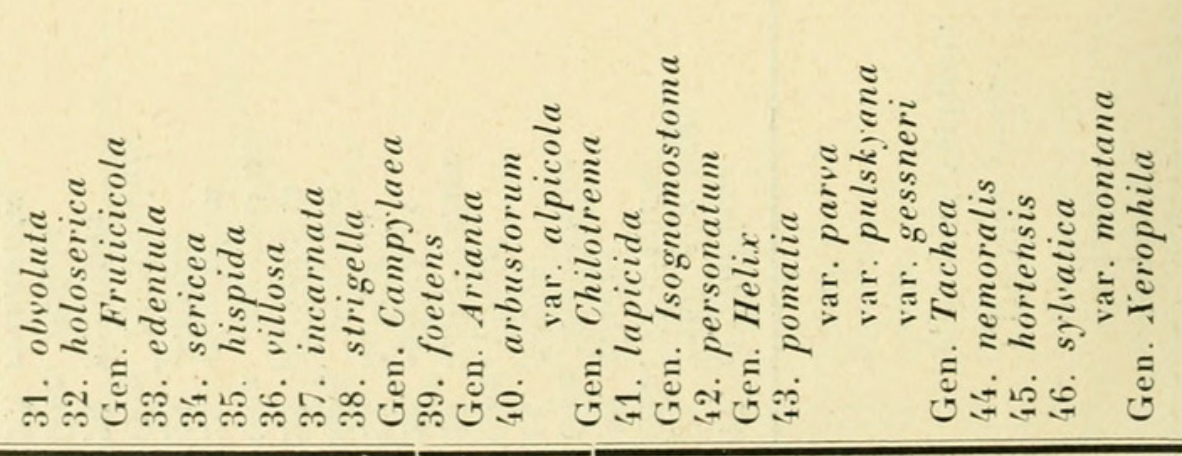 \\
\hline
\end{tabular}




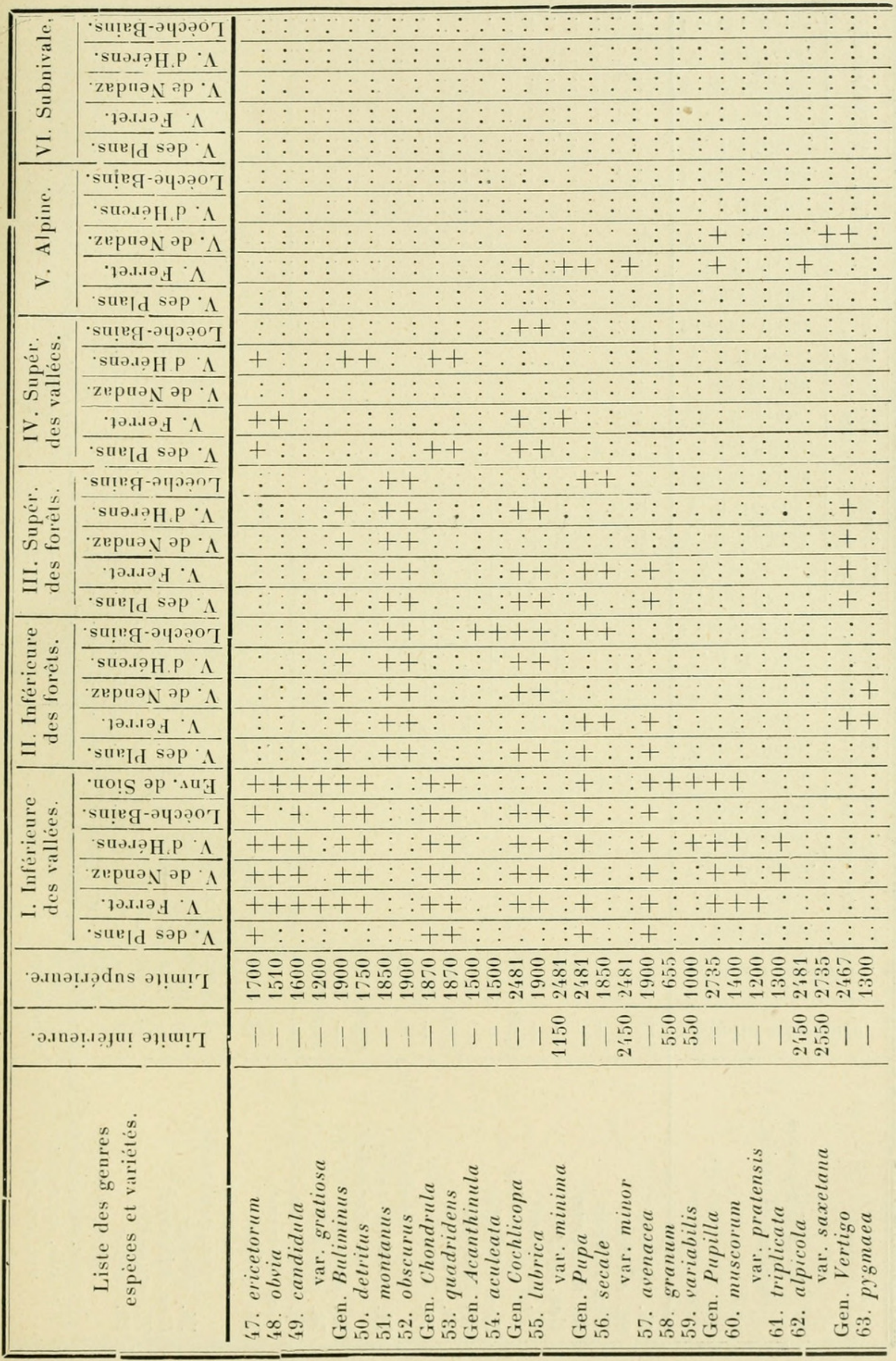




\begin{tabular}{|c|c|c|}
\hline \multirow{5}{*}{ 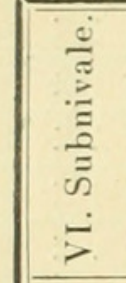 } & & $:: \quad:$ \\
\hline & & $::: \quad: \quad$ \\
\hline & & $:::::::$ \\
\hline & & $::::::::::::$ : \\
\hline & & 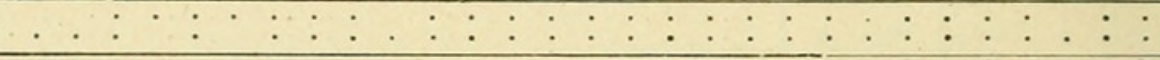 \\
\hline \multirow{5}{*}{$\frac{\frac{3}{2}}{2}$} & & 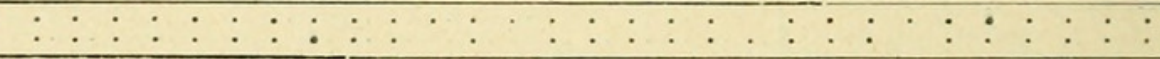 \\
\hline & $\Lambda$ & $::::$ : : : : : : : : : : : : : : . : : : \\
\hline & $\Lambda$ & 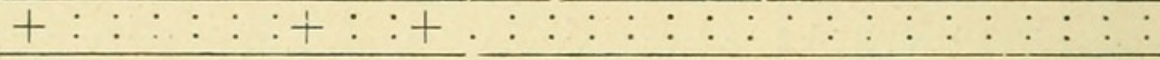 \\
\hline & & 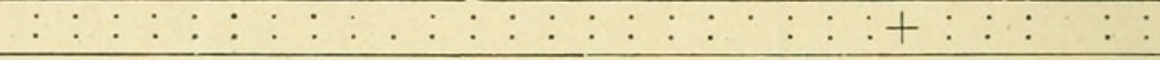 \\
\hline & & 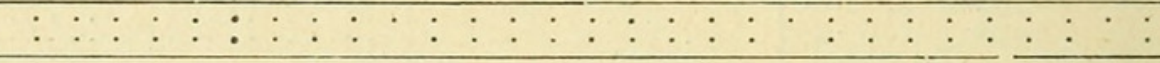 \\
\hline \multirow{5}{*}{ 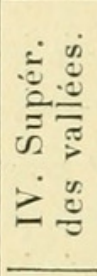 } & & 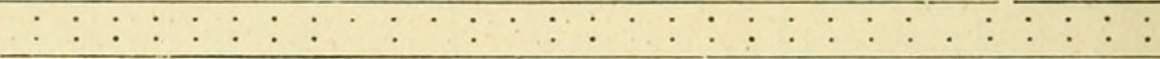 \\
\hline & & 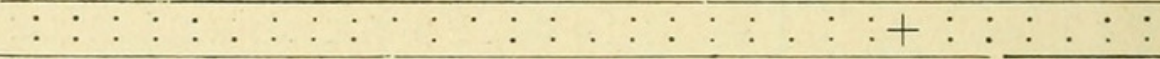 \\
\hline & $\Lambda$ & 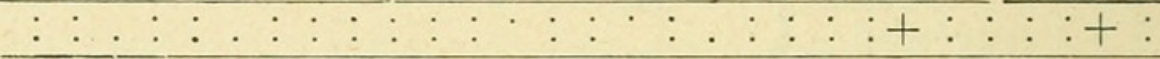 \\
\hline & & 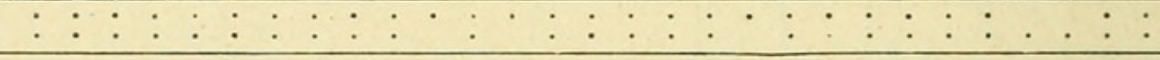 \\
\hline & 1 & 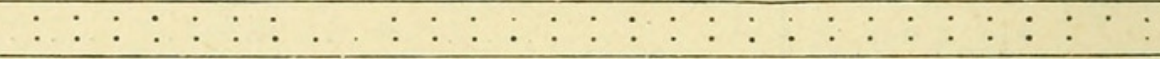 \\
\hline \multirow{5}{*}{ 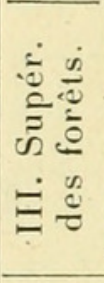 } & & 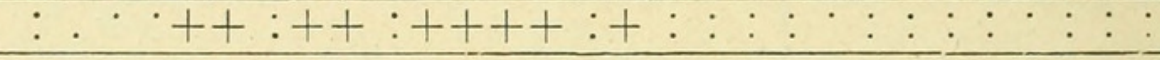 \\
\hline & & 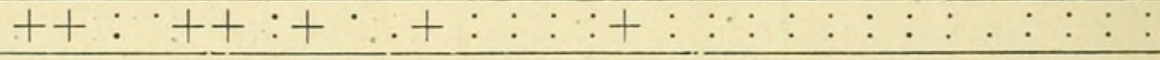 \\
\hline & & 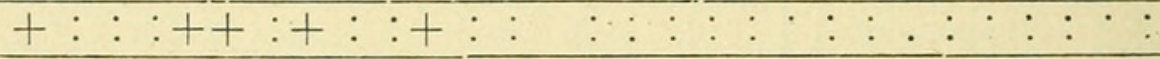 \\
\hline & & $+:::+++++:+::+\cdot+:: \vdots: \vdots:: \vdots: \vdots$ \\
\hline & $\Lambda$ & $+::::::++++:: \cdot:+:::::: \vdots:: \vdots:$ \\
\hline \multirow{5}{*}{ 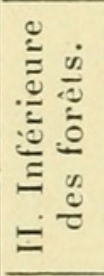 } & & 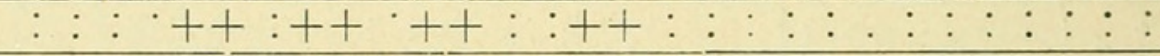 \\
\hline & & $::::++++::+::::++: \vdots::: \vdots::: \vdots:$ \\
\hline & & $::::++:+::+:::+++: \cdot:: \vdots::: \vdots:$ \\
\hline & & $::::++:++:+::: \circ++:::: \vdots::::: \vdots$ \\
\hline & & $::::++:++:+:::+++::::: \cdot:::::$ \\
\hline \multirow{6}{*}{ 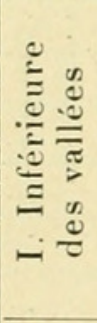 } & & $:++::::: \cdot:::::^{\prime}:++1::++:+:: \vdots$ \\
\hline & & 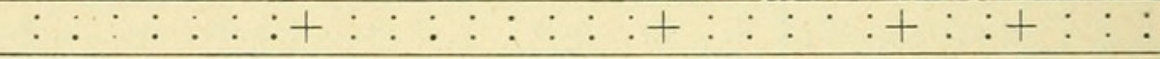 \\
\hline & $\Lambda$. & $::++:::::::::::::++::++::+::+$ \\
\hline & $\cdot z e p$ & 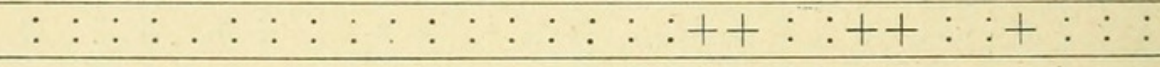 \\
\hline & & $:::::::::::: \cdot::::++:+:+::++::$ \\
\hline & & 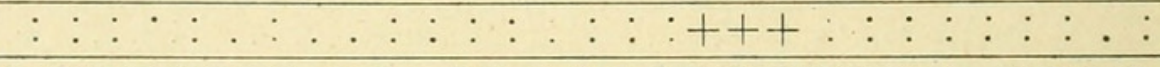 \\
\hline \multicolumn{2}{|c|}{ 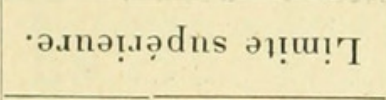 } & 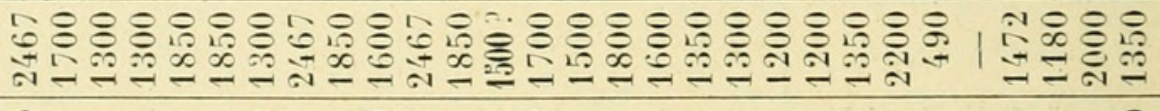 \\
\hline \multicolumn{2}{|c|}{ 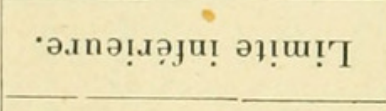 } & 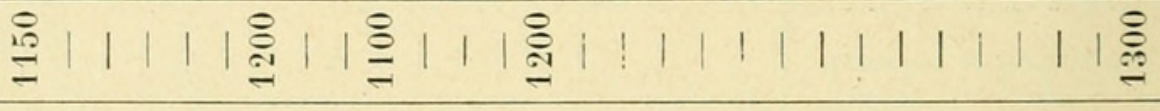 \\
\hline & 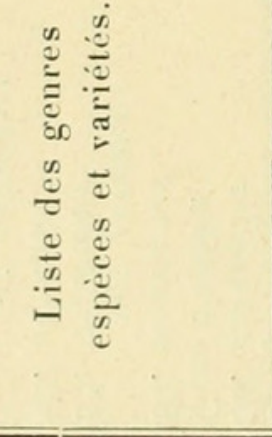 & 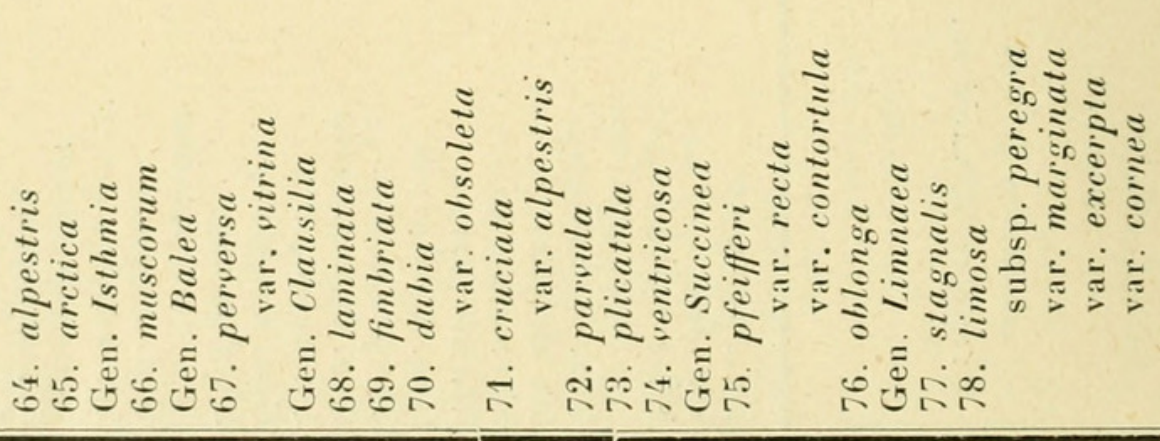 \\
\hline
\end{tabular}




\begin{tabular}{|c|c|c|}
\hline \multirow{5}{*}{ 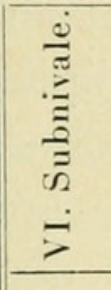 } & & $::$ \\
\hline & & $::::::^{\cdot}:$ \\
\hline & $\mathrm{Ce}$ & : : : : : : : : : : : : : : \\
\hline & & 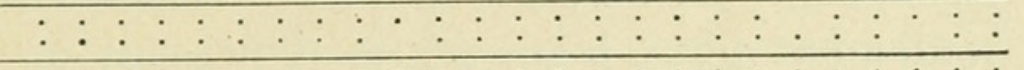 \\
\hline & & $::::::::$ : : : : : : : \\
\hline \multirow{5}{*}{$\begin{array}{l}\frac{3}{2} \\
\frac{2}{2} \\
>\end{array}$} & & 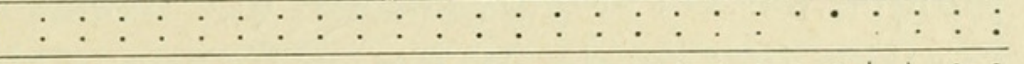 \\
\hline & & 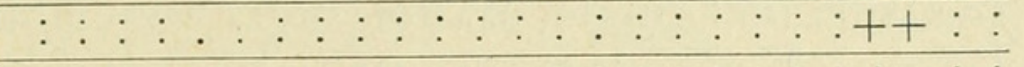 \\
\hline & ze: & 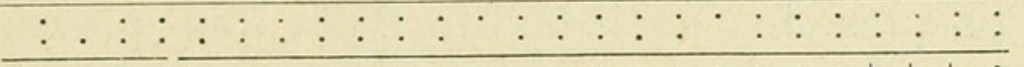 \\
\hline & & 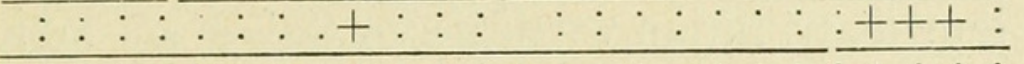 \\
\hline & & 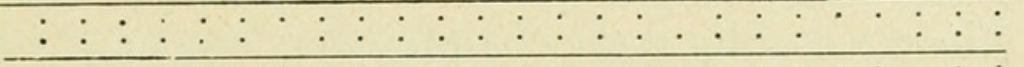 \\
\hline \multirow{5}{*}{ 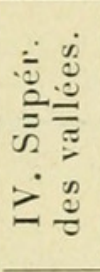 } & & 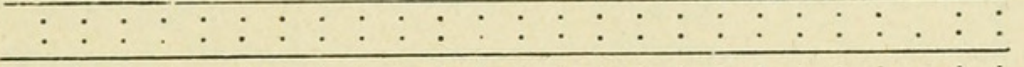 \\
\hline & $\Lambda$ & 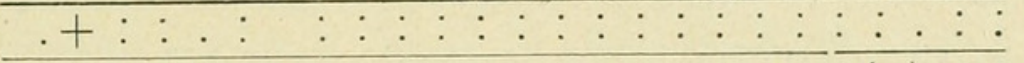 \\
\hline & $\cdot \mathrm{ze}$ & 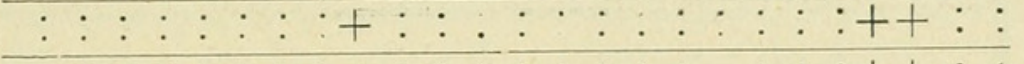 \\
\hline & & $:::::::::::::::::: 0^{\circ}::++$ \\
\hline & & 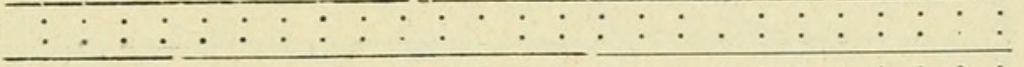 \\
\hline \multirow{5}{*}{ 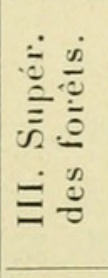 } & & 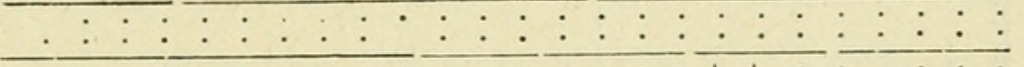 \\
\hline & & 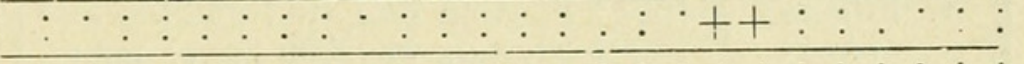 \\
\hline & 'ze & 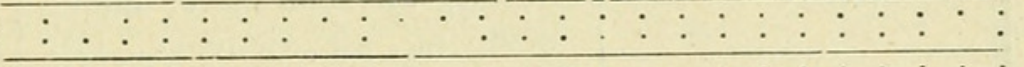 \\
\hline & & 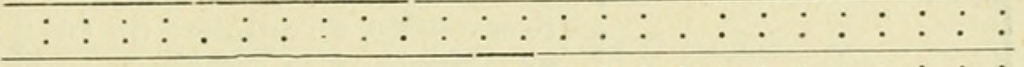 \\
\hline & & $::::::: \vdots::::::::$ : \\
\hline \multirow{5}{*}{ 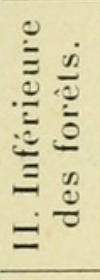 } & & 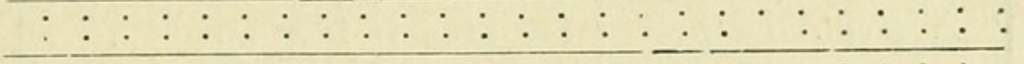 \\
\hline & - si & $::::::::: \cdot:::::::::$ : \\
\hline & ze & $::::::::_{0}::::::{ }^{\prime}$ \\
\hline & & 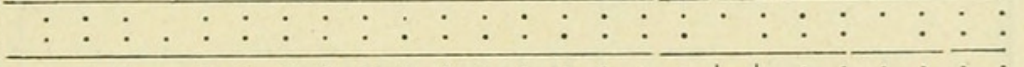 \\
\hline & 'st & 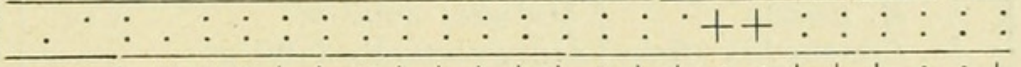 \\
\hline \multirow{6}{*}{ 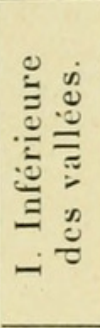 } & & $::+:: \cdot++:++++:++::+$ \\
\hline & & 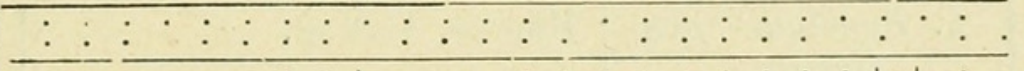 \\
\hline & $\cdot \mathrm{si}$ & $::: .:::+$ \\
\hline & zep & $: \quad: .: 0^{\circ}::++:$ \\
\hline & 7 & $+::+++:$ \\
\hline & & $\therefore:$ \\
\hline \multicolumn{2}{|c|}{ 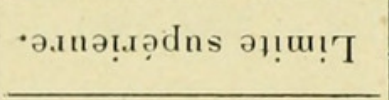 } & 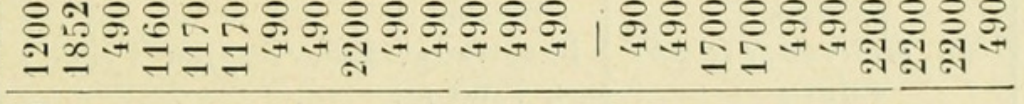 \\
\hline \multicolumn{2}{|c|}{ 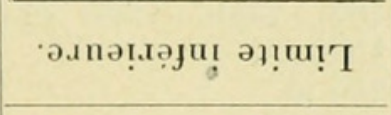 } & $\underline{E}=|1| 1|1| 1|1| 1 \mid 1$ \\
\hline & 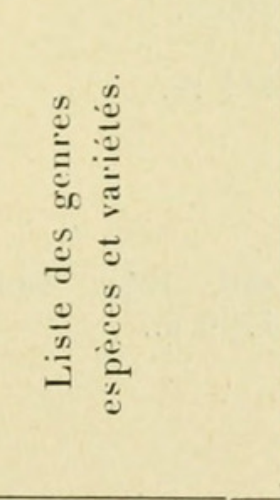 & 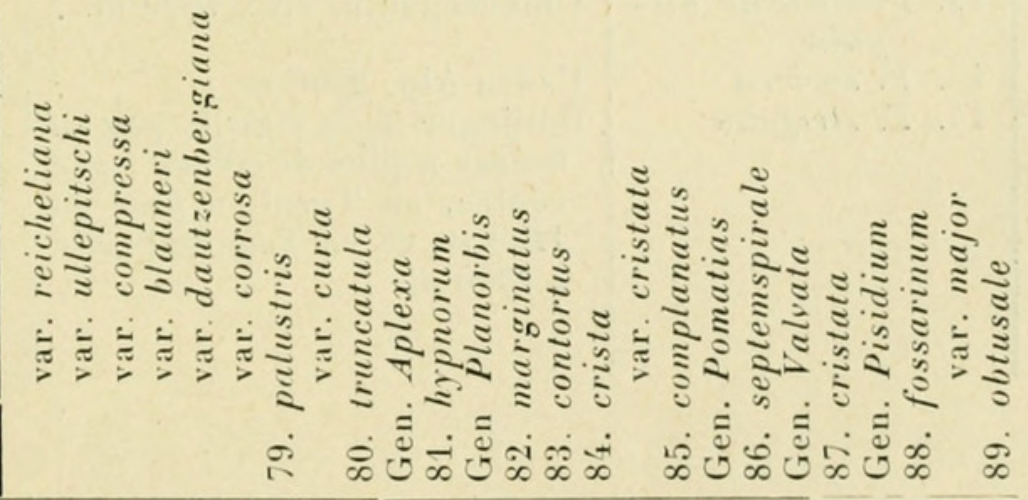 \\
\hline
\end{tabular}


Pour terminer, il m'a paru intéressant de faire brièvement une comparaison entre les maxima hypsométriques indiqués par M. Bollinger (op. cit.) et le résultat de mes recherches, seulement pour les espèces dépassant en altitude le record indiqué par cet auteur. On sait que dans son intéressante dissertation inaugurale, M. Bollinger rassemble à propos des Mollusques des environs de Bâle, une quantilé de données sur leur distribution verticale en Suisse ou mème ailleurs, de manière à établir pour chaque espèce un maximum observé.

\begin{tabular}{|c|c|c|}
\hline Espèces. & Maxima de Bollinger. & $\begin{array}{c}\text { Nouveaux matériaux } \\
\text { du Valais. }\end{array}$ \\
\hline $\begin{array}{l}\text { 1. Vitrina pellu- } \\
\text { cida } \\
\text { 2. Hyalina depres- } \\
\text { sa } \\
\text { 3. H. glabra } \\
\text { 4. H. nitens } \\
\text { 5. Euconulus ful- } \\
\text { vus } \\
\text { 6. Punctum pyg- } \\
\text { maeum } \\
\text { 7. Patula rotun- } \\
\text { data } \\
\text { 8. P. ruderata } \\
\text { 9. Pyramidula ru- } \\
\text { pestris } \\
\text { 10. Helicodonta ob- } \\
\text { voluta } \\
\text { 11. H. holoserica } \\
\text { 12. Fruticicola his- } \\
\text { pida } \\
\text { 13. F. sericea } \\
\text { 14. F. strigella }\end{array}$ & 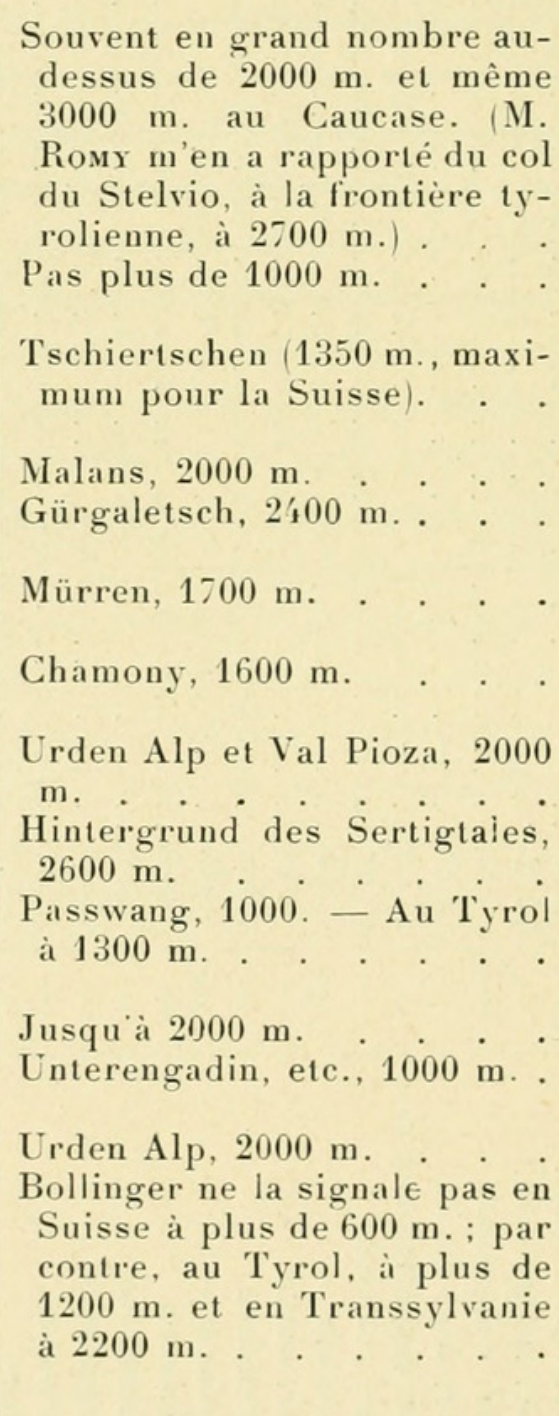 & $\begin{array}{l}2481 \mathrm{~m} . \\
2800-2900 \mathrm{~m} . \\
1600 \mathrm{~m} . \\
1400,1600,1700 \text { et } \\
1850 \mathrm{~m} . \\
2200 \mathrm{~m} . \\
2467 \text { et } 2481 \mathrm{~m} . \\
1800,2200,2467 \text { et } \\
2481 \mathrm{~m} . \\
1800 \mathrm{~m} . \\
2100 \mathrm{et} 2200 \mathrm{~m} . \\
2735 \text { et } 2800-2900 \mathrm{~m} . \\
1200,1300,1350 \text { et } \\
1500 \mathrm{~m} . \\
2200 \mathrm{~m} . \\
1300 \mathrm{~m} . \\
2481 \mathrm{~m} .\end{array}$ \\
\hline
\end{tabular}


Espèces.

Maxima de Bollinger.

Nouveaux matériaux des Alpes.

15. Chilotrema lapicida

16. Isognostoma personatum

17. Tachea hortensis

18. T. nemoralis

19. T. sylvatica

20. Xerophila obvia

21. Buliminus detritus

22. B. obscurus

23. Chondrula quadridens

24. Cochlicopa lubrica

25. Pupa secale

26. P. avenacea

27. Balea perversa

28. Clausilia laminata

29. C. fimbriata

30. C. cruciata

31. C. dubia
"In den Alpen hält sich mehr an die untern Hänge der Täler». Jura, $1000 \mathrm{~m}$. Calfeisental. $1560 \mathrm{~m}$. . . . .

Jusqu'à $1400 \mathrm{~m}$ $1600 \mathrm{~m}$.

"In den Alpen beschränkı sie sich auf die breiteren und tiefer gelegєnen Talgründe. ” Jura, $900 \mathrm{~m}$.

Piémont, $1000 \mathrm{~m}$.

Gemmi, $2300 \mathrm{~m} \cdot$

Unter Engadin, $1200 \mathrm{~m}$. .

Engadine, $1500 \mathrm{~m}$. . . .

Mürren, $1700 \mathrm{~m}$. . . . .

Tschiertschen, $1340 \mathrm{~m}$. et au Tyrol (Ahrental), $1500 \mathrm{~m}$.

Bergell, 1350 jusqu'à $2250 \mathrm{~m}$.

Mürren, $1700 \mathrm{~m}$.

Alpstein, $1850 \mathrm{~m}$. . . $1900 \mathrm{~m}$

Hinterrhein, $1600 \mathrm{~m}$. . . 1800 et $1850 \mathrm{~m}$.

Mürren, $1700 \mathrm{~m}$. . . . $1850 \mathrm{~m}$.

Jura, $1000 \mathrm{~m}$. . . . . $1600 \mathrm{~m}$.

$1500 \mathrm{~m}$. . . . . 1600 et $1700 \mathrm{~m}$.

Urden Alp, $2000 \mathrm{~m}$. . . . $2467 \mathrm{~m}$. 
Bollinger, G. 1909. Zur Gastropodenfauna yon Basel und Umgebung. Basel.

Brot, A. 1896? Notes manuscrites en marge du Catalogue de Charpentifr (1837), dans l'exemplaire qui se trouve actuellement déposé à la Bibliothèque du Musée d'Histoire naturelle de Genève.

Charpentier, J. de. 1837. Catalogue des Mollusques terrestres el fluviatiles de la Suisse. Mém. Soc. helv. Sc. nat., vol. 1, p. 1-28, pl. I et II.

Chessin, S. 1887-1890. Die Mollustienfauna Oesterreich-Ungarns und der Schweiz. Nürnberg, 858 p., 528 fig.

Godet, P. 1907. Calalogue des Mollusques du canton de Neuchâtel et des régions limitrophes des cantons de Berne, Vaud et Fribourg. Bull. Soc. neuchàteloise Sc. nat., vol. 34, p. 97-158, pl. I et II.

Lenman, W. 1911. Untersuchungen über die Fauna des Sigriswylgrates. Rev. Suisse Zool., vol. 19, pl. 63-115 (2 fig. et 1 carte malac.).

Payot, V. 1864. Erpétologie, malacologie et paléontologie des environs du Mont-Blanc. Ann. Soc. impér. Agric., Hist. nat. et Arts utiles Lyon, extrait (Malac., p. 23-53).

Piaget, J. 1911. Mollusques recueillis dans la région supérieure du Val d'Hérens. Rameau de Sapin, vol. 45, p. 30-32, 40 et 46-47.

lo. 1912. Note sur trois variétés nouvelles de Mollusques suisses. Journ. Conchyl., vol. 59 (1911), p. 333-340, 7 fig.

Roffiann, F. 1868. Mollusques terrestres et flusiatiles recueillis en Suisse. Ann. Soc. malac. Belgique, vol. 3, p. 65-84, pl. I, fig. 1-9.

Stolt, O. 1899. Beiträge zur Kenntnis der schweizerischen Mollustienfauna. Vierteljahrs. Naturf. Gesellsch. Zürich, vol. 44, p. 1-87.

ID. 1901. Ueber xerothermische Relikten in der Schweizerfauna der Wirbellosen. Festschrift Geogr. Ethnogr. Gesellsch. Zürich, 1900-1901, p. 145-208. 
Studer, S. 1820. Systematisches Verzeichnis der bis jetzt beliannt gewordenen Schweizer-Conchylien. Berne, $32 \mathrm{p}$.

Vax dex Broeck, E. 1875. Notes sur une excursion scientifique en Suisse, août-septembre 1875. Rapport présenté à la Société malacologique de Belgique. Ann. Soc. malac. Belgique, vol. 10, p. cxxıx à cLxvi (Liste des Mollusques vipants recueillis pendant l'excursion : p. cLxm à cLxvi). 


\section{$2 \mathrm{BHL}$ Biodiversity Heritage Library}

1913. "Malacologie alpestre." Revue suisse de zoologie 21, 439-575. https://doi.org/10.5962/bhl.part.37158.

View This Item Online: https://www.biodiversitylibrary.org/item/40668

DOI: https://doi.org/10.5962/bhl.part.37158

Permalink: https://www.biodiversitylibrary.org/partpdf/37158

\section{Holding Institution}

MBLWHOI Library

\section{Sponsored by}

MBLWHOI Library

\section{Copyright \& Reuse}

Copyright Status: NOT_IN_COPYRIGHT

This document was created from content at the Biodiversity Heritage Library, the world's largest open access digital library for biodiversity literature and archives. Visit BHL at https://www.biodiversitylibrary.org. 